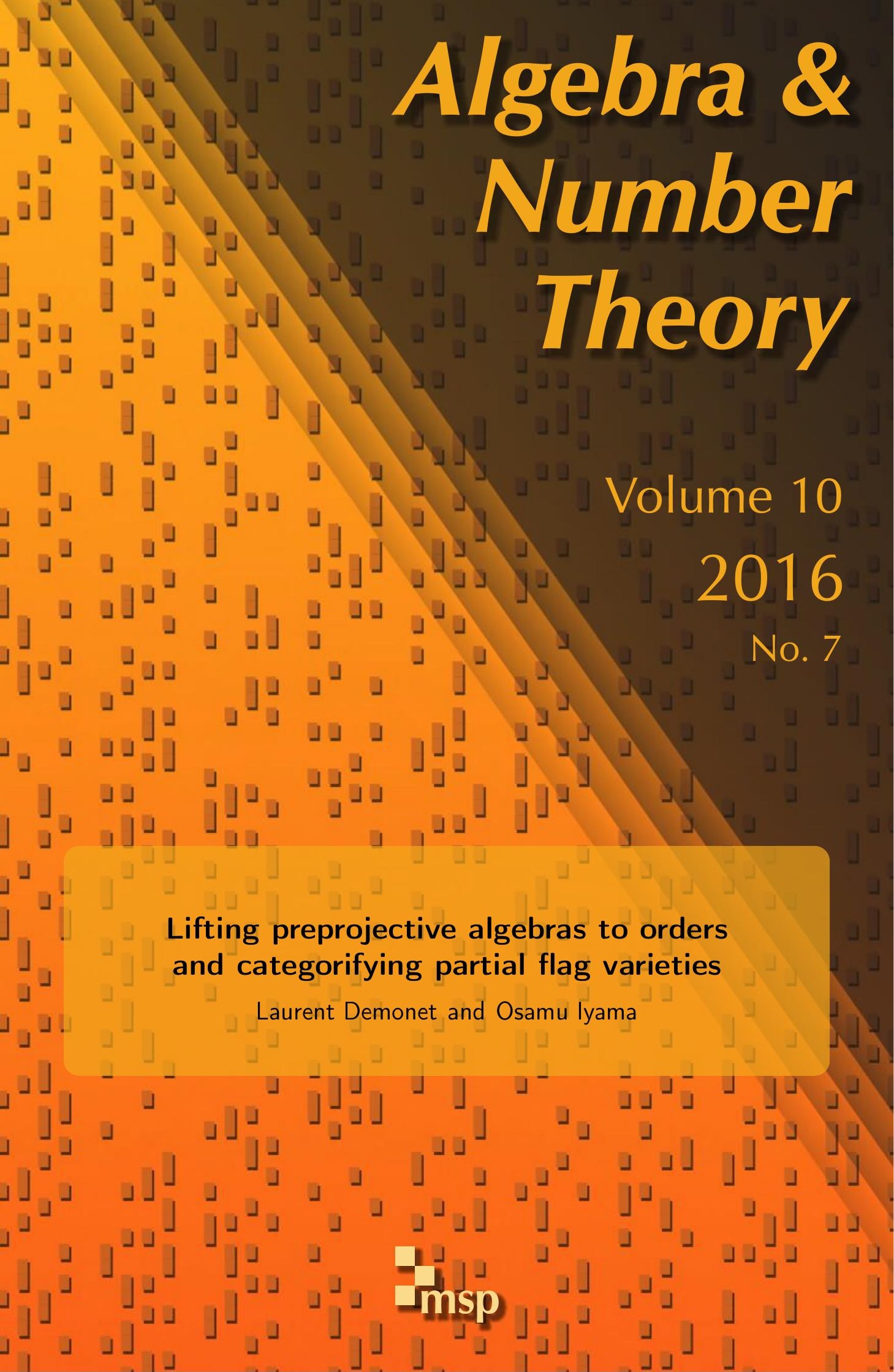




\title{
Lifting preprojective algebras to orders and categorifying partial flag varieties
}

\author{
Laurent Demonet and Osamu lyama
}

\begin{abstract}
We describe a categorification of the cluster algebra structure of multihomogeneous coordinate rings of partial flag varieties of arbitrary Dynkin type using Cohen-Macaulay modules over orders. This completes the categorification of Geiss, Leclerc and Schröer by adding the missing coefficients. To achieve this, for an order $A$ and an idempotent $e \in A$, we introduce a subcategory $\mathrm{CM}_{e} A$ of $\mathrm{CM} A$ and study its properties. In particular, under some mild assumptions, we construct an equivalence of exact categories $\left(\mathrm{CM}_{e} A\right) /[A e] \cong \operatorname{Sub} Q$ for an injective $B$-module $Q$, where $B:=A /(e)$. These results generalize work by Jensen, King and $\mathrm{Su}$ concerning the cluster algebra structure of the Grassmannian $\mathrm{Gr}_{m}\left(\mathbb{C}^{n}\right)$.
\end{abstract}

1. Introduction

2. Main results

3. Results on exact categories

4. Equivalences arising from torsion pairs on exact categories

5. Equivalences arising from orders and their idempotents

6. Cluster algebra structure on coordinate rings of partial flag varieties

Acknowledgement

1578

References

\section{Introduction}

Geiss, Leclerc and Schröer [Geiss et al. 2008] introduced a cluster algebra structure on some subalgebra $\tilde{\mathcal{A}}$ of the multihomogeneous coordinate ring $\mathbb{C}[\mathcal{F}]$ of the partial flag variety $\mathcal{F}=\mathcal{F}(\Delta, J)$ corresponding to a Dynkin diagram $\Delta$ and a set $J$ of vertices of $\Delta$. They proved that $\tilde{\mathcal{A}}=\mathbb{C}[\mathcal{F}]$ in type $A$, and conjectured that the

Demonet was partially supported by JSPS Grant-in-Aid for Young Scientist (B) 26800008. Iyama was partially supported by JSPS Grant-in-Aid for Scientific Research (B) 24340004, (B) 16H03923, (C) 23540045, and (S) 15H05738.

MSC2010: primary 16G30; secondary 13F60, 16G10, 16G50, 18E10, 18E30.

Keywords: orders, Cohen-Macaulay modules, finite-dimensional algebras, preprojective algebras, categorification, cluster algebras, partial flag varieties, exact categories. 
equality holds after an appropriate localization for any Dynkin type (see Section 6 for more details). This structure generalizes previously known cases of Grassmannians, introduced for $\mathrm{Gr}_{2}\left(\mathbb{C}^{n}\right)$ by Fomin and Zelevinsky [2003] (see also [Berenstein et al. 2005]) and generalized by Scott [2006] for $\mathrm{Gr}_{k}\left(\mathbb{C}^{n}\right)$.

In the same paper, Geiss, Leclerc and Schröer introduced a partial categorification of this cluster algebra structure on $\tilde{\mathcal{A}}$. A crucial role is played by the preprojective algebra $\Pi$ of type $\Delta$ and a certain full subcategory Sub $Q_{J}$ of $\bmod \Pi$ which is Frobenius and stably 2-Calabi-Yau. More precisely, they introduced a cluster character $\tilde{\varphi}$ : Sub $Q_{J} \rightarrow \tilde{\mathcal{A}}$ which gives a bijection

$\left\{\right.$ reachable indecomposable rigid objects in Sub $\left.Q_{J}\right\} / \cong$

$\stackrel{1-1}{\longleftrightarrow}\{$ cluster variables and coefficients of $\tilde{\mathcal{A}}\} \backslash\left\{\Delta_{j} \mid j \in J\right\}$,

where $\Delta_{j}$ is the prinicipal generalized minor corresponding to $j \in J$.

One of the aim of this paper is to look for a stably 2-Calabi-Yau category extending Sub $Q_{J}$ whose reachable indecomposable rigid objects correspond to cluster variables and all coefficients of $\tilde{\mathcal{A}}$. Jensen, King and Su [Jensen et al. 2016] achieved this in the case of classical Grassmannians (i.e., $\Delta=A_{n}$ for $n \geq 0$ and $\# J=1$ ) by using orders (see also [Baur et al. 2016] for an interpretation in terms of dimer models). In this article, we extend their method to any arbitrary Dynkin diagram $\Delta$ and arbitrary set of vertices $J$.

Throughout the introduction, for simplicity, let $R:=k \llbracket t \rrbracket$ be the formal power series ring over an arbitrary field $k$. For an $R$-order $A$ (i.e., an $R$-algebra that is free of finite rank as an $R$-module), we denote by CM $A$ the category of Cohen-Macaulay modules over $A$ (i.e., $A$-modules that are free of finite rank over $R$ ). For an idempotent $e \in A$, we define

$$
\mathrm{CM}_{e} A:=\{X \in \mathrm{CM} A \mid e X \in \operatorname{proj}(e A e)\} .
$$

We prove the following result:

Theorem A (Theorems 6.10 and 6.12). Let $\Delta$ be a Dynkin diagram, and $J$ be a set of vertices of $\Delta$. Then, there exist a $\mathbb{C} \llbracket t \rrbracket-o r d e r ~ A$, an idempotent $e \in A$ such that $\mathrm{CM}_{e} A$ is Frobenius and stably 2-Calabi-Yau, and a cluster character $\psi: \mathrm{CM}_{e} A \rightarrow \tilde{\mathcal{A}}$ such that

(a) $\psi$ induces a bijection between

- isomorphism classes of reachable indecomposable rigid objects of $\mathrm{CM}_{e} A$,

- cluster variables and coefficients of $\tilde{\mathcal{A}}$,

(b) $\psi$ induces a bijection between

- isomorphism classes of reachable basic cluster tilting objects of $\mathrm{CM}_{e} A$,

- clusters of $\tilde{\mathcal{A}}$.

Moreover, it commutes with mutation of cluster tilting objects and mutation of clusters. 
To prove Theorem A, we generalize techniques introduced by Jensen, King and $\mathrm{Su}$ [Jensen et al. 2016] for Grassmannians in type $A$ (see also [Demonet and Luo 2016b] for Grassmannians of 2-dimensional planes in type A). Meanwhile, we need to prove general results on orders.

The study of Cohen-Macaulay modules (also known as lattices) over orders is a classical subject in representation theory. We refer to [Auslander 1978; Curtis and Reiner 1981; Leuschke and Wiegand 2012; Simson 1992; Yoshino 1990] for a general background on this subject. We also refer to [Amiot et al. 2015; Araya 1999; Demonet and Luo 2016a; 2016b; Herschend et al. 2014; de Thanhoffer de Völcsey and Van den Bergh 2010; Iyama and Takahashi 2013; Kajiura et al. 2007; 2009; Keller and Reiten 2008] for recent results about connections with tilting theory and cluster categories.

We consider an $R$-order $A$ and an idempotent $e \in A$ such that $B:=A /(e)$ is finite-dimensional over $k$. Let $K:=k((t))$ be the fraction field of $R$, let $U:=$ $\operatorname{Hom}_{A}\left(B, A e \otimes_{R}(K / R)\right)$ and let Sub $U$ be the category of $B$-submodules of objects $U^{n}$ for $n \geq 0$. We consider the exact full subcategory

$$
\bmod _{e} A:=\{X \in \bmod A \mid e X \in \operatorname{proj}(e A e)\}
$$

of $\bmod A$. Under this setting, we prove the following generalization of a result of [Jensen et al. 2016].

Theorem $\mathbf{B}$ (Theorem 2.2). Assume that Ae is injective in $\mathrm{CM}_{e} A$ and has injective dimension at most 1 in $\bmod _{e} A$. Then $U$ is injective in $\bmod B$ and there is an equivalence of exact categories

$$
B \otimes_{A}-:\left(\mathrm{CM}_{e} A\right) /[A e] \stackrel{\sim}{\longrightarrow} \operatorname{Sub} U .
$$

In particular, if $e$ and $g$ are idempotents of an $R$-order $A$ such that $B=A /(e)$ is finite-dimensional and $A e \cong \operatorname{Hom}_{R}(g A, R)$ as left $A$-modules, then the hypotheses of Theorem $\mathrm{B}$ are satisfied and $U$ is the injective $B$-module corresponding to the idempotent $g$ (see Theorem 2.1). Let us give a motivating example:

Example. For $n \geq 1$, we consider the pair $(A, e)$ defined as

$$
A:=\left[\begin{array}{cc}
R & R \\
\left(t^{n}\right) & R
\end{array}\right] \text { and } e:=\left[\begin{array}{ll}
1 & 0 \\
0 & 0
\end{array}\right] .
$$

We have $A e \cong \operatorname{Hom}_{R}((1-e) A, R)$ and $B=A /(e) \cong k[t] /\left(t^{n}\right)$. So according to Theorem B,

$$
\left(\mathrm{CM}_{e} A\right) /[A e] \cong \operatorname{Sub} U=\bmod B .
$$

Notice that here $\mathrm{CM}(e A e)=\operatorname{proj}(e A e)$, so $\mathrm{CM}_{e} A=\mathrm{CM} A$. We can illustrate this fact by drawing the Auslander-Reiten quivers of $\mathrm{CM} A$ and $\bmod B$ : 


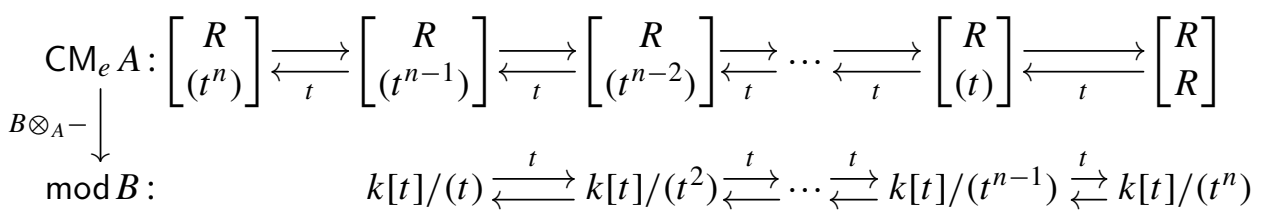

where projective-injective objects are leftmost and rightmost in the first row and only rightmost in the second row. On the other objects, the Auslander-Reiten translation acts as the identity.

As an application of Theorem B, we get the following, which is fundamental for Theorem A:

Corollary C (Corollary of Theorem 2.1). Let $B$ be a finite-dimensional selfinjective k-algebra. We define a Gorenstein order $A$ over $R=k \llbracket t \rrbracket$ and an idempotent e of $A$ by

$$
A:=B \otimes_{k}\left[\begin{array}{cc}
R & R \\
t R & R
\end{array}\right] \text { and } e:=\left[\begin{array}{ll}
1 & 0 \\
0 & 0
\end{array}\right] .
$$

Then we have an equivalence of exact categories $\left(\mathrm{CM}_{e} A\right) /[A e] \cong \bmod B$, which induces a triangle equivalence $\underline{\mathrm{CM}}_{e} A \cong \underline{\bmod } B$ between stable categories.

Additionally, we prove a categorical version of Theorem B in the context of exact categories:

Theorem D (Theorem 4.7). Let $\mathcal{E}$ be an exact category which is Hom-finite over a field $k$. We suppose that

- $(\mathcal{A}, \mathcal{B})$ and $(\mathcal{B}, \mathcal{C})$ are torsion pairs in $\mathcal{E}$

- $\mathcal{E}$ has enough projective objects, which belong to $\mathcal{C}$;

- there exists a projective object $P$ in $\mathcal{E}$ which is injective in $\mathcal{C}$ and satisfies $\mathcal{A}=$ add $P$

- $\mathcal{B}$ is an abelian category whose exact structure is compatible with that of $\mathcal{E}$.

Then, there is an equivalence of exact categories

$$
\mathcal{C} /[\mathcal{A}] \stackrel{\sim}{\longrightarrow} \operatorname{Sub} U
$$

where $U$ is an (explicitly constructed) injective object of $\mathcal{B}$.

Notice that we need and we prove more general versions of Theorems B and D, with more technical hypotheses and more precise conclusions.

The structure of this paper is as follows. In Section 2, we explain main results about orders over an arbitrary complete discrete valuation ring $R$, and provide more general and more detailed versions of Theorem B. We also give a systematic way to construct pairs $(A, e)$ satisfying the hypotheses of Theorem $\mathrm{B}$ for a prescribed 
algebra $B$. The results of Section 2 are proven in Section 5. In Section 3, we recall the basics of exact categories and we give sufficient conditions for an ideal quotient category $\mathcal{E} /[\mathcal{F}]$ of an exact category $\mathcal{E}$ by a subcategory $\mathcal{F}$ of projective-injective objects to inherit the exact structure of $\mathcal{E}$. In Section 4, we give extended versions of Theorem D. Finally, in Section 6, we prove Theorem A.

\section{Main results}

2A. Orders. Let $R$ be a complete discrete valuation ring and $K$ be its field of fractions. Let $A$ be an $R$-order, i.e., an $R$-algebra which is free of finite rank as an $R$-module. We denote by f.l. $A$ the full subcategory of $\bmod A$ consisting of finite-length $A$-modules, or equivalently $A$-modules which are of finite length over $R$. Recall that, in this context, a finitely generated $A$-module $X$ is (maximal) Cohen-Macaulay if the following equivalent conditions are satisfied:

(i) $X$ is free (of finite rank) as an $R$-module;

(ii) $\operatorname{Hom}_{A}$ (f.I. $\left.A, X\right)=0$, or equivalently $\operatorname{soc} X=0$;

(iii) $\operatorname{Ext}_{A}^{1}\left(X, \operatorname{Hom}_{R}(A, R)\right)=0$, or equivalently, $\operatorname{Ext}_{A}^{i}\left(X, \operatorname{Hom}_{R}(A, R)\right)=0$ for any $i>0$.

We denote by $\mathrm{CM} A$ the exact full subcategory of $\bmod A$ consisting of CohenMacaulay $A$-modules. Since $A$ is an $R$-order, both $A$ and $\operatorname{Hom}_{R}(A, R)$ are in CM $A$. It is clear from (ii) that (f.I. $A, \mathrm{CM} A$ ) is a torsion pair in $\bmod A$, which can be seen as coming from the cotilting $A$-module $\operatorname{Hom}_{R}(A, R)$.

For an idempotent $e$ of $A$, we consider a full subcategory of CM $A$ :

$$
\mathrm{CM}_{e} A:=\{X \in \mathrm{CM} A \mid e X \in \operatorname{proj}(e A e)\} .
$$

This is clearly closed under extensions, and hence forms an exact category naturally. If $e A e$ is a hereditary order (i.e., gl.dim $e A e=1$ ), then $\mathrm{CM}_{e} A=\mathrm{CM} A$ holds because $\mathrm{CM}(e A e)=\operatorname{proj}(e A e)$.

Our first main theorem, generalizing [Jensen et al. 2016], is the following one:

Theorem 2.1. Let $A$ be an $R$-order, and e be an idempotent of $A$. Assume that the following conditions are satisfied:

- $B:=A /(e)$ satisfies length ${ }_{R} B<\infty$.

- There is an idempotent $g \in A$ such that $\operatorname{add} A e=\operatorname{add}_{\operatorname{Hom}}(g A, R)$ as A-modules.

Then the following assertions hold:

(a) We have an equivalence of exact categories

$$
F=B \otimes_{A}-:\left(\mathrm{CM}_{e} A\right) /[A e] \stackrel{\sim}{\longrightarrow} \operatorname{Sub} Q_{g},
$$


where $Q_{g}$ is the injective $B$-module associated with the image of the idempotent $g$ in $B$.

(b) A quasi-inverse of $F$ is $\operatorname{Hom}_{R}\left(\Omega_{A} \operatorname{Hom}_{R}(-, K / R), R\right)$, where $\Omega_{A}$ is the syzygy over A.

We assume in addition that the following hypotheses hold:

- There exists an idempotent $f \in A$ such that $\operatorname{add} A f=\operatorname{add}_{H^{\prime}}(e A, R)$ as A-modules.

- eAe is a Gorenstein order.

Then the following conclusions hold:

(c) The module $Q_{g}$ is a projective $B$-module satisfying add $Q_{g}=\operatorname{add} B f$.

(d) If $A \in \mathrm{CM}_{e} A$, then Sub $Q_{g}=\operatorname{Sub} B$.

We suppose in addition that $A$ and $\operatorname{Hom}_{R}(A, R)$ are in $\mathrm{CM}_{e} A$.

(e) The order A is Gorenstein if and only if $B$ is Iwanaga-Gorenstein of dimension at most 1, i.e., inj. $\operatorname{dim}_{B} B \leq 1$ and inj.dim $B_{B} \leq 1$.

(f) If the conditions in (e) are satisfied, then we have triangle equivalences

$$
\underline{\mathrm{CM}_{e}} A \cong \underline{\mathrm{Sub}} Q_{g}=\underline{\mathrm{Sub}} B,
$$

where $\underline{\mathrm{CM}}_{e} A:=\left(\mathrm{CM}_{e} A\right) /[A]$ and $\underline{\mathrm{Sub}} B=(\operatorname{Sub} B) /[B]$.

Corollary $\mathrm{C}$ presented in the introduction is an immediate consequence of Theorem 2.1 as it is immediate that $A e \cong \operatorname{Hom}_{R}(g A, R)$ for $g:=1-e$ in that case. In this paper, a more general version of Theorem 2.1 plays an important role. Again let $A$ be an $R$-order and $e$ an idempotent of $A$. Let

$$
\bmod _{e} A:=\{X \in \bmod A \mid e X \in \operatorname{proj}(e A e)\} .
$$

We consider the following conditions:

(E1) $A e$ is injective in $\mathrm{CM}_{e} A$, or equivalently, $\operatorname{Ext}_{A}^{1}\left(\mathrm{CM}_{e} A, A e\right)=0$;

(E2) $\operatorname{Ext}_{\bmod _{e} A}^{2}\left(\bmod _{e} A, A e\right)=0$;

$(\mathrm{E} 2)^{+} \operatorname{Ext}_{A}^{2}\left(\bmod _{e} A, A e\right)=0$.

We recall the definition of the $\operatorname{Ext}_{\mathcal{E}}^{i}$ in Section 3 for exact categories $\mathcal{E}$. For a

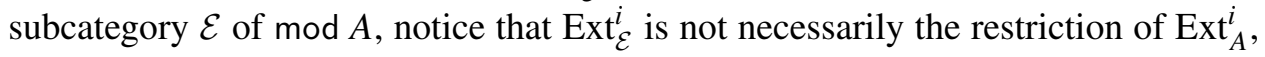
except for $i=1$. In Lemma 5.7, we prove the following implications:

- We have $(\mathrm{E} 2)^{+} \Rightarrow(\mathrm{E} 2)$.

- If $A e=\operatorname{Hom}_{R}(g A, R)$ for some idempotent $g \in A$, then (E1) and (E2) ${ }^{+}$are satisfied.

- If (E1) is satisfied and $A \in \mathrm{CM}_{e} A$, then (E2) ${ }^{+}$is satisfied. 
Theorem 2.1 follows from the next result:

Theorem 2.2. Let $A$ be an $R$-order and e an idempotent of $A$ such that $B:=A /(e)$ satisfies length ${ }_{R} B<\infty$. Then:

(a) $(\operatorname{add} A e, \bmod B)$ and $\left(\bmod B, \mathrm{CM}_{e} A\right)$ are torsion pairs in $\bmod _{e} A$.

(b) Let $\mathcal{E}_{1}:=\left\{X \in \bmod _{e} A \mid \operatorname{Ext}_{A}^{1}(X, A e)=0\right\}$. We have an equivalence

$$
B \otimes_{A}-: \mathcal{E}_{1} /[A e] \stackrel{\sim}{\longrightarrow} \bmod B .
$$

If (E1) is satisfied, then the following assertion holds:

(c) Let $U:=\operatorname{Hom}_{A}\left(B, A e \otimes_{R}(K / R)\right) \in \bmod B$, where $K$ is the fraction field of $R$. The equivalence (2-1) restricts to an equivalence

$$
B \otimes_{A}-:\left(\mathrm{CM}_{e} A\right) /[A e] \stackrel{\sim}{\longrightarrow} \operatorname{Sub} U .
$$

If (E1) and (E2) are satisfied, then the following assertions hold:

(d) $U$ is an injective $B$-module.

(e) (2-1) and (2-2) are equivalences of exact categories, where $\mathcal{E}_{1} /[\mathrm{Ae}]$ and $\left(\mathrm{CM}_{e} A\right) /[A e]$ inherit canonically the exact structure of $\mathcal{E}_{1}$ and $\mathrm{CM}_{e} A$ (see Section 3).

(f) The exact categories $\mathcal{E}_{1}, \mathrm{CM}_{e} A, \bmod _{e} A$ and $\operatorname{Sub} U$ have enough projective objects and enough injective objects.

(g) Let $P$ be a projective cover of $\operatorname{soc} U$ as a $B$-module. Then, we have the equality $\mathcal{E}_{1}=\left\{X \in \bmod _{e} A \mid \operatorname{Hom}_{A}(P, X)=0\right\}$.

2B. Change of orders. We give a systematic method to construct pairs of orders and their idempotents which satisfy the conditions (E1) and (E2).

Let $A$ be an $R$-order, $e$ an idempotent of $A$ and $B$ a factor algebra of $A /(e)$. We suppose that the following two conditions are satisfied:

(C1) length ${ }_{R} B<\infty$;

(C2) $B \in \operatorname{Sub}\left(A e \otimes_{R}(K / R)\right)$.

Let $\bmod _{e}^{B} A$ be the category of all $X \in \bmod A$ such that there exists an exact sequence

$$
0 \rightarrow P \rightarrow X \rightarrow Y \rightarrow 0
$$

with $P \in \operatorname{add} A e$ and $Y \in \bmod B$. Let $\mathrm{CM}_{e}^{B} A:=\mathrm{CM} A \cap \bmod _{e}^{B} A$ and consider the condition:

(C3) $\operatorname{Ext}_{A}^{1}\left(\mathrm{CM}_{e}^{B} A, A e\right)=0$. 
We will construct a new order $A^{\prime}$ under this setting. Thanks to (C2), there is a monomorphism $\iota: B \hookrightarrow\left(A e \otimes_{R}(K / R)\right)^{\oplus \ell}$. Applying $A e^{\oplus \ell} \otimes_{R}-$ to the exact sequence $0 \rightarrow R \rightarrow K \rightarrow K / R \rightarrow 0$ and taking a pullback via $\iota$, we get a short exact sequence

$$
0 \rightarrow P \rightarrow \widetilde{B} \rightarrow B \rightarrow 0
$$

with $P \in$ add $A e$ and $\widetilde{B} \in \mathrm{CM} A$. We clearly have $\widetilde{B} \in \mathrm{CM}_{e}^{B} A$. Using (C3), one can check $\tilde{B}$ is independent of the choice of $\iota$ up to a direct summand in add $A e$ (see Theorem 4.1(a)). Let

$$
W:=A e \oplus \widetilde{B} \quad \text { and } \quad A^{\prime}:=\operatorname{End}_{A}(W) .
$$

We can regard naturally $e$ as an idempotent of $A^{\prime}$. Notice that $A^{\prime}$ is uniquely defined up to Morita equivalence.

Theorem 2.3. We assume that (C1), (C2) and (C3) hold. Then the following assertions hold:

(a) We have a canonical isomorphism $B \cong A^{\prime} /(e)$ of $R$-algebras.

(b) We have (E1) holds, that is, $\operatorname{Ext}_{A^{\prime}}^{1}\left(\mathrm{CM}_{e} A^{\prime}, A^{\prime} e\right)=0$, and $(E 2)^{+}$holds, that is, $\operatorname{Ext}_{A^{\prime}}^{2}\left(\bmod _{e} A^{\prime}, A^{\prime} e\right)=0$.

(c) Let $U:=\operatorname{Hom}_{A^{\prime}}\left(B, A^{\prime} e \otimes_{R}(K / R)\right) \in \bmod B$. Then $U$ is an injective $B$-module and we have an equivalence of exact categories

$$
B \otimes_{A^{\prime}}-:\left(\mathrm{CM}_{e} A^{\prime}\right) /\left[A^{\prime} e\right] \stackrel{\sim}{\longrightarrow} \operatorname{Sub} U .
$$

(d) The class of short exact sequences of $\bmod A$ with three terms in $\bmod _{e}^{B} A$ gives the structure of an exact category on $\bmod _{e}^{B} A$. The same holds for $\mathrm{CM}_{e}^{B} A$. For these structures, the functors

$\operatorname{Hom}_{A}(W,-): \bmod A \rightarrow \bmod A^{\prime} \quad$ and $\quad W \otimes_{A^{\prime}}-: \bmod A^{\prime} \rightarrow \bmod A$ induce quasi-inverse equivalences of exact categories between $\bmod _{e}^{B} A$ and $\bmod _{e} A^{\prime}$ on the one hand, and between $\mathrm{CM}_{e}^{B} A$ and $\mathrm{CM}_{e} A^{\prime}$ on the other hand.

(e) We have a commutative diagram

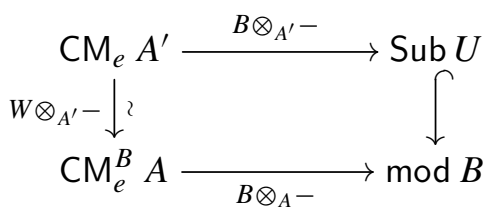

where all functors induce isomorphisms of $\mathrm{Ext}^{1}$ and the left side is an equivalence of exact categories for the exact structure on $\mathrm{CM}_{e}^{B}$ A given in (d).

Let us finally introduce a simple criterion for $(\mathrm{C} 1),(\mathrm{C} 2)$ and $(\mathrm{C} 3)$ to be satisfied: 
Lemma 2.4. Let $A$ be an $R$-order, $e$ an idempotent of $A$ and $B$ a factor algebra of $A /(e)$. Let us assume that there exists an idempotent $g \in A$ such that $A e \cong$ $\operatorname{Hom}_{R}(g A, R)$. Then (C3) holds. Moreover, if (C1) holds, then (C2) holds if and only if $(1-g) \operatorname{soc} B=0$.

We will prove Lemma 2.4 at the end of Section 5C.

In the rest of this subsection we give an example illustrating Theorem 2.3. Let $B=\Pi$ be the preprojective algebra of type $A_{3}$ over a field $k$. In other terms

$$
\Pi=k\left(1 \underset{\beta_{1}}{\stackrel{\alpha_{1}}{\gtrless}} 2 \underset{\beta_{2}}{\stackrel{\alpha_{2}}{\longrightarrow}} 3\right) /\left(\alpha_{1} \beta_{1}, \alpha_{2} \beta_{2}-\beta_{1} \alpha_{1}, \beta_{2} \alpha_{2}\right) .
$$

This algebra can also be realized as the following subquotient of the matrix algebra $M_{3}(k[\varepsilon])$ :

$$
\Pi=\left[\begin{array}{ccc}
k[\varepsilon] /(\varepsilon) & k[\varepsilon] /(\varepsilon) & k[\varepsilon] /(\varepsilon) \\
(\varepsilon) /\left(\varepsilon^{2}\right) & k[\varepsilon] /\left(\varepsilon^{2}\right) & k[\varepsilon] /(\varepsilon) \\
\left(\varepsilon^{2}\right) /\left(\varepsilon^{3}\right) & (\varepsilon) /\left(\varepsilon^{2}\right) & k[\varepsilon] /(\varepsilon)
\end{array}\right] .
$$

Let us define $R:=k \llbracket t \rrbracket$ and $S:=R[\varepsilon]$. The $R$-order considered in Corollary $\mathrm{C}$ is

$$
A:=\left[\begin{array}{cccccc}
S /(\varepsilon) & S /(\varepsilon) & S /(\varepsilon) & S /(\varepsilon) & S /(\varepsilon) & S /(\varepsilon) \\
(\varepsilon) /\left(\varepsilon^{2}\right) & S /\left(\varepsilon^{2}\right) & S /(\varepsilon) & (\varepsilon) /\left(\varepsilon^{2}\right) & S /\left(\varepsilon^{2}\right) & S /(\varepsilon) \\
\left(\varepsilon^{2}\right) /\left(\varepsilon^{3}\right) & (\varepsilon) /\left(\varepsilon^{2}\right) & S /(\varepsilon) & \left(\varepsilon^{2}\right) /\left(\varepsilon^{3}\right) & (\varepsilon) /\left(\varepsilon^{2}\right) & S /(\varepsilon) \\
(t) /(t \varepsilon) & (t) /(t \varepsilon) & (t) /(t \varepsilon) & S /(\varepsilon) & S /(\varepsilon) & S /(\varepsilon) \\
(t \varepsilon) /\left(t \varepsilon^{2}\right) & (t) /\left(t \varepsilon^{2}\right) & (t) /(t \varepsilon) & (\varepsilon) /\left(\varepsilon^{2}\right) & S /\left(\varepsilon^{2}\right) & S /(\varepsilon) \\
\left(t \varepsilon^{2}\right) /\left(t \varepsilon^{3}\right) & (t \varepsilon) /\left(t \varepsilon^{2}\right) & (t) /(t \varepsilon) & \left(\varepsilon^{2}\right) /\left(\varepsilon^{3}\right) & (\varepsilon) /\left(\varepsilon^{2}\right) & S /(\varepsilon)
\end{array}\right] .
$$

In Figure 1, we draw the Auslander-Reiten quiver of $\mathrm{CM}_{e} A$, with notations

$$
\begin{aligned}
i j & :=\left(t^{i} \varepsilon^{j}\right) /\left(t^{i} \varepsilon^{j+1}\right), \\
i j & :=\left(t^{i} \varepsilon^{j}\right) /\left(t^{i} \varepsilon^{j+2}\right), \\
i j-i j & :=\{(p, q) \in i j \times i j \mid p-q \in t \cdot i j\} .
\end{aligned}
$$

Thus, the identity of $S$ induces a map $i j \rightarrow i^{\prime} j^{\prime}$ if and only if $(j, i) \geq\left(j^{\prime}, i^{\prime}\right)$ for the lexicographic order and analogous rules can be computed for $i j$. All arrows are induced by multiplications by an element of $S$, which is \pm 1 when it is not specified.

Let $e_{3}, e_{2}, e_{1}, g_{1}, g_{2}$ and $g_{3}$ be the idempotents corresponding, in this order, to the rows of the matrix. They satisfy

$$
A e_{i} \cong \operatorname{Hom}_{R}\left(g_{i} A, R\right) \text { and } A g_{i} \cong \operatorname{Hom}_{R}\left(e_{i} A, R\right)
$$




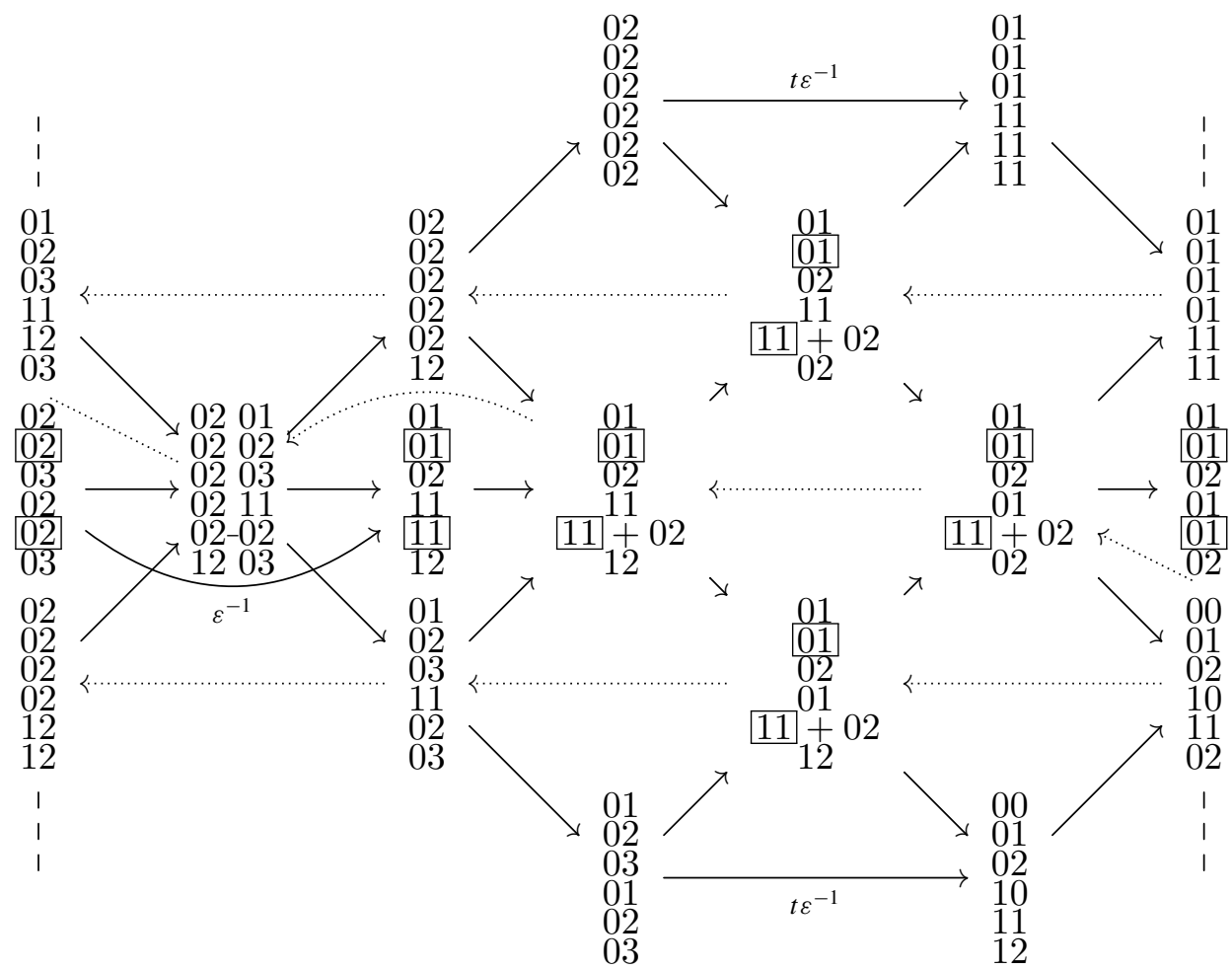

Figure 1. Auslander-Reiten quiver of $\mathrm{CM}_{e} A$.

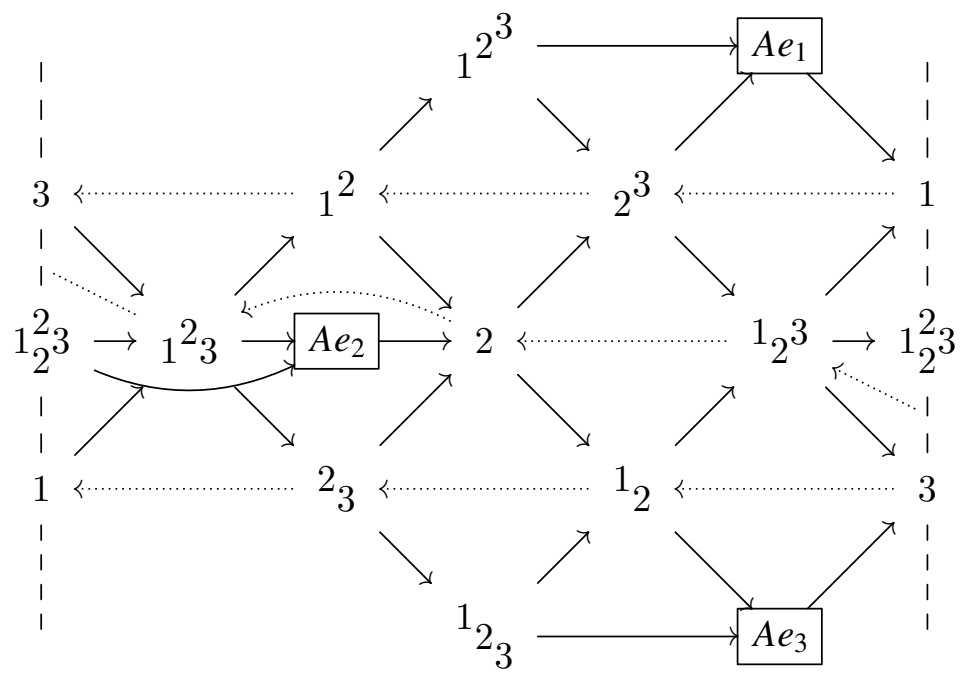

Figure 2. Auslander-Reiten quiver of $\mathrm{CM}_{e} A$. Objects are represented by their image by $F$ except objects of add $A e$. 
as $A$-modules. We fix the idempotent $e=e_{1}+e_{2}+e_{3}$. According to Corollary $\mathrm{C}$, we have an equivalence of exact categories

$$
\left(\mathrm{CM}_{e} A\right) /[A e] \cong \bmod \Pi \text {. }
$$

In Figure 2, we draw the Auslander-Reiten quiver of $\mathrm{CM}_{e} A$, replacing objects which are not in add $A e$ by their image by $F$ in Sub $U=\bmod \Pi$ (here $U=\Pi$ ). We obtain the Auslander-Reiten quiver of mod $\Pi$ by removing framed objects. The general relation between Auslander-Reiten quivers of $\mathrm{CM}_{e} A$ and $\mathrm{Sub} U$ will be discussed in [Demonet and Iyama $\geq 2016$ ].

We explain the way to compute the minimal preimage of an object of Sub $U$ by $F$ in this example. First, we know that preimages of simple modules $S_{i}$ are coradicals of indecomposable direct summands of $A e$. Thus, we find

$$
F\left(S_{1}^{\circ}\right) \cong S_{1}, \quad F\left(S_{2}^{\circ}\right) \cong S_{2}, \quad F\left(S_{3}^{\circ}\right) \cong S_{3},
$$

where

$$
S_{1}^{\circ}=\left[\begin{array}{c}
S /(\varepsilon) \\
S /(\varepsilon) \\
S /(\varepsilon) \\
S /(\varepsilon) \\
(t) /(t \varepsilon) \\
(t) /(t \varepsilon)
\end{array}\right], \quad S_{2}^{\circ}=\left[\begin{array}{c}
S /(\varepsilon) \\
S /\left(\varepsilon^{2}\right) \\
(\varepsilon) /\left(\varepsilon^{2}\right) \\
(t) /(t \varepsilon) \\
(t, \varepsilon) /\left(\varepsilon^{2}\right) \\
(t \varepsilon) /\left(t \varepsilon^{2}\right)
\end{array}\right], \quad S_{3}^{\circ}=\left[\begin{array}{c}
S /(\varepsilon) \\
(\varepsilon) /\left(\varepsilon^{2}\right) \\
\left(\varepsilon^{2}\right) /\left(\varepsilon^{3}\right) \\
(t) /(t \varepsilon) \\
(t \varepsilon) /\left(t \varepsilon^{2}\right) \\
\left(\varepsilon^{2}\right) /\left(\varepsilon^{3}\right)
\end{array}\right] .
$$

Let us calculate the preimage $X^{\circ}$ of ${ }_{1}^{2} 3$ by $F$. There exists a pullback diagram

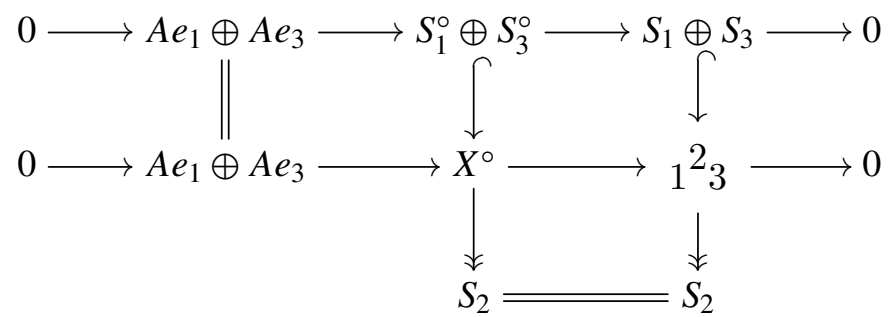

which permits us to get

$$
X^{\circ}=\left[\begin{array}{cr}
S /(\varepsilon) & S /(\varepsilon) \\
S /(\varepsilon) & (\varepsilon) /\left(\varepsilon^{2}\right) \\
S /(\varepsilon) & \left(\varepsilon^{2}\right) /\left(\varepsilon^{3}\right) \\
S /(\varepsilon) & (t) /(t \varepsilon) \\
S /(\varepsilon) & -(\varepsilon) /\left(\varepsilon^{2}\right) \\
(t) /(t \varepsilon) & \left(\varepsilon^{2}\right) /\left(\varepsilon^{3}\right)
\end{array}\right]
$$



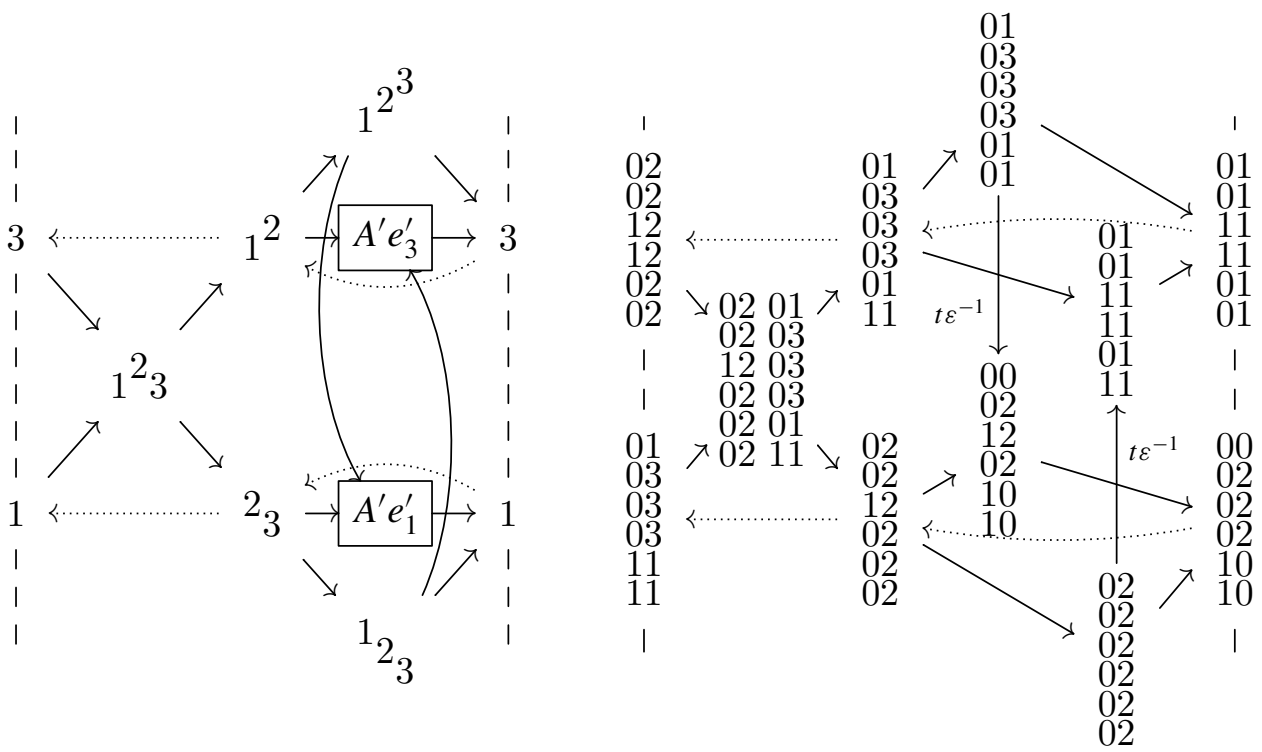

Figure 3. Auslander-Reiten quiver of $\mathrm{CM}_{e^{\prime}} A^{\prime}$. In the left diagram, objects are represented by their image by $F$ except objects of add $A^{\prime} e^{\prime}$.

where

$$
\left[S /(\varepsilon)-(\varepsilon) /\left(\varepsilon^{2}\right)\right]:=\left\{(x, \varepsilon y) \in S /(\varepsilon) \times(\varepsilon) /\left(\varepsilon^{2}\right) \mid x-y \in t \cdot S /(\varepsilon)\right\} .
$$

Now, we apply Theorem 2.3. Let $e^{\prime}:=e_{1}+e_{3}$ and $B^{\prime}:=\Pi /\left(\beta_{1} \alpha_{1}\right)$. As a $B$-module,

$$
B^{\prime} \cong{ }^{1}{ }_{3} \oplus{ }_{1}{ }_{3} \oplus{ }_{1} 2^{3}
$$

Thanks to Lemma $2.4, B^{\prime}$ and $e^{\prime}$ satisfy the hypotheses of Theorem 2.3. Then, keeping notations of this subsection, we have

$$
W=A e_{1} \oplus A e_{3} \oplus A g_{1} \oplus X^{\circ} \oplus A g_{3} .
$$

Then, $A^{\prime}:=\operatorname{End}_{A}(W)$ is easy to compute:

$$
A^{\prime}=\left[\begin{array}{cccccc}
S /(\varepsilon) & S /(\varepsilon) & S /(\varepsilon) & S /(\varepsilon) & S /(\varepsilon) & S /(\varepsilon) \\
\left(\varepsilon^{2}\right) /\left(\varepsilon^{3}\right) & S /(\varepsilon) & S /(\varepsilon) & S /(\varepsilon) & \left(\varepsilon^{2}\right) /\left(\varepsilon^{3}\right) & \left(\varepsilon^{2}\right) /\left(\varepsilon^{3}\right) \\
\left(t \varepsilon^{2}\right) /\left(t \varepsilon^{3}\right) & (t) /(t \varepsilon) & S /(\varepsilon) & (t) /(t \varepsilon) & \left(\varepsilon^{2}\right) /\left(\varepsilon^{3}\right) & \left(\varepsilon^{2}\right) /\left(\varepsilon^{3}\right) \\
\left(\varepsilon^{2}\right) /\left(\varepsilon^{3}\right) & (t) /(t \varepsilon) & S /(\varepsilon) & S /(\varepsilon) & \left(\varepsilon^{2}\right) /\left(\varepsilon^{3}\right) & \left(\varepsilon^{2}\right) /\left(\varepsilon^{3}\right) \\
(t) /(t \varepsilon) & S /(\varepsilon) & S /(\varepsilon) & S /(\varepsilon) & S /(\varepsilon) & S /(\varepsilon) \\
(t) /(t \varepsilon) & (t) /(t \varepsilon) & S /(\varepsilon) & S /(\varepsilon) & (t) /(t \varepsilon) & S /(\varepsilon)
\end{array}\right]
$$


where

$$
[S /(\varepsilon)-S /(\varepsilon)]:=\{(x, y) \in S /(\varepsilon) \times S /(\varepsilon) \mid x-y \in t \cdot S /(\varepsilon)\} .
$$

Thanks to Theorem 2.3, we have $\left(\mathrm{CM}_{e^{\prime}} A^{\prime}\right) /\left[A e^{\prime}\right]$ is equivalent to the subcategory of $\bmod \Pi$ consisting of modules whose socle is supported at vertices 1 and 3 . To illustrate this fact, we give two representations of the Auslander-Reiten quiver of $\mathrm{CM}_{e^{\prime}} A^{\prime}$ in Figure 3.

2C. Notations. In this paper, if $f: X \rightarrow Y$ and $g: Y \rightarrow Z$ are two morphisms in a category, we write $f g: X \rightarrow Z$ for the composed morphism.

Let $\mathcal{A} b$ be the category of abelian groups. For an additive category $\mathcal{A}$, an $\mathcal{A}$-module is a contravariant additive functor $F: \mathcal{A} \rightarrow \mathcal{A} b$. We say that an $\mathcal{A}$-module $F$ is finitely generated if there exists an epimorphism of $\mathcal{A}$-modules $\operatorname{Hom}_{\mathcal{A}}(\mathcal{A}, X) \rightarrow F$ for some $X \in \mathcal{A}$.

\section{Results on exact categories}

The aim of this section is to study ideal quotient categories $\mathcal{E} /[\mathcal{F}]$ of an exact category $\mathcal{E}$ by a full subcategory $\mathcal{F}$ consisting of projective-injective objects. More precisely, we study conditions for $\mathcal{E} /[\mathcal{F}]$ to inherit the exact structure of $\mathcal{E}$. In particular, we prove that it is the case if and only if admissible monomorphisms and epimorphisms are mapped to categorical monomorphisms and epimorphisms by the canonical projection $\mathcal{E} \rightarrow \mathcal{E} /[\mathcal{F}]$. This is a particular case of Theorem 3.6.

3A. Preliminaries about exact categories. We recall here main definitions and elementary results about exact categories. We consider an additive category $\mathcal{E}$ endowed with a family $\mathcal{S}$ of pairs of morphisms $(f, g)$ of $\mathcal{E}$, where $f$ is a kernel of $g$ and $g$ is a cokernel of $f$. We denote such a pair by

$$
0 \rightarrow X \stackrel{f}{\longrightarrow} Y \stackrel{g}{\longrightarrow} Z \rightarrow 0,
$$

and for $(f, g) \in \mathcal{S}$, we call $(f, g)$ an admissible short exact sequence, $f$ an admissible monomorphism and $g$ an admissible epimorphism. We call $(\mathcal{E}, \mathcal{S})$ an exact category if it satisfies the following axioms due to Quillen [1973] and modified by Keller [1990, Appendix A]:

$(\operatorname{Ex} 0) \mathcal{S}$ is stable under isomorphisms and contains split short exact sequences of the form

$$
0 \rightarrow X \stackrel{\left[\mathrm{id}_{X} 0\right]}{\longrightarrow} X \oplus Z \stackrel{\left[\begin{array}{c}
0 \\
\mathrm{id}_{Z}
\end{array}\right]}{\longrightarrow} Z \rightarrow 0 .
$$

(Ex1) The composition of two admissible epimorphisms is an admissible epimorphism. 
$(E x 1)^{\text {op }}$ The composition of two admissible monomorphisms is an admissible monomorphism.

(Ex2) For any admissible short exact sequence

$$
0 \rightarrow X \stackrel{f}{\longrightarrow} Y \stackrel{g}{\longrightarrow} Z \rightarrow 0
$$

and morphism $v: Z^{\prime} \rightarrow Z$, we can form a pullback diagram, i.e., a commutative diagram of the form

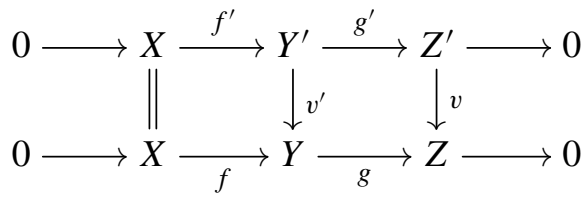

where the first row is an admissible short exact sequence.

$(\text { Ex2) })^{\text {op }}$ For any admissible short exact sequence

$$
0 \rightarrow X \stackrel{f}{\longrightarrow} Y \stackrel{g}{\longrightarrow} Z \rightarrow 0
$$

and morphism $u: X \rightarrow X^{\prime}$, we can form a pushout diagram, i.e., a commutative diagram of the form

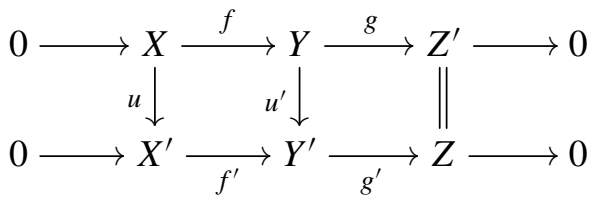

where the second row is an admissible short exact sequence.

We often write $\mathcal{E}$ instead of $(\mathcal{E}, \mathcal{S})$ when we consider only one exact structure on $\mathcal{E}$. When not specified, we use the terms short exact sequence, monomorphism and epimorphism for admissible short exact sequence, admissible monomorphism, admissible epimorphism, respectively. In contrast, we use categorical monomorphism or categorical epimorphism for a monomorphism or epimorphism which is not necessarily admissible.

We will use freely the following easy facts about exact categories:

- In (Ex2), we have the admissible short exact sequence

$$
0 \rightarrow Y^{\prime} \stackrel{\left[v^{\prime} g^{\prime}\right]}{\longrightarrow} Y \oplus Z^{\prime} \stackrel{\left[\begin{array}{c}
g \\
-v
\end{array}\right]}{\longrightarrow} Z \rightarrow 0 .
$$

- In (Ex2), if $v$ is an admissible epimorphism, then so is $v^{\prime}$ and $\operatorname{Ker} v=\left(\operatorname{Ker} v^{\prime}\right) g^{\prime}$.

- In (Ex2), if $v$ is an admissible monomorphism, then so is $v^{\prime}$ and Coker $v^{\prime}=$ $g(\operatorname{Coker} v)$. 
- In (Ex2) $)^{\mathrm{op}}$, we have the admissible short exact sequence

$$
0 \rightarrow X \stackrel{[u f]}{\longrightarrow} X^{\prime} \oplus Y \stackrel{\left[\begin{array}{c}
f^{\prime} \\
-u^{\prime}
\end{array}\right]}{\longrightarrow} Y^{\prime} \rightarrow 0 .
$$

- In (Ex2) ${ }^{\mathrm{op}}$, if $u$ is an admissible epimorphism, then so is $u^{\prime}$ and $\operatorname{Ker} u^{\prime}=(\operatorname{Ker} u) f$.

- In (Ex2) ${ }^{\mathrm{op}}$, if $u$ is an admissible monomorphism, then so is $u^{\prime}$ and Coker $u=$ $f^{\prime}\left(\operatorname{Coker} u^{\prime}\right)$.

- If a morphism is an admissible monomorphism and an admissible epimorphism, then it is an isomorphism.

- If, in a morphism of short exact sequences, the left and right components are both admissible monomorphisms or epimorphisms, then the middle one is as well.

- In (Ex2) and (Ex2) ${ }^{\mathrm{op}}$, the diagrams are uniquely determined up to unique isomorphisms.

Let us recall the following definition:

Definition 3.1. A functor $F$ between exact categories $(\mathcal{E}, \mathcal{S})$ and $\left(\mathcal{E}^{\prime}, \mathcal{S}^{\prime}\right)$ is exact if $F(\mathcal{S}) \subset \mathcal{S}^{\prime}$. An object $X \in \mathcal{E}$ is projective if $\operatorname{Hom}_{\mathcal{E}}(X,-)$ is exact, and injective if $\operatorname{Hom}_{\mathcal{E}}(-, X)$ is exact. We say that $\mathcal{E}$ has enough injective objects if for any $X \in \mathcal{E}$ there exists a short exact sequence $0 \rightarrow X \rightarrow I \rightarrow Y \rightarrow 0$ in $\mathcal{S}$ such that $I$ is injective. We say that $\mathcal{E}$ has enough projective objects if for any $X \in \mathcal{E}$ there exists a short exact sequence $0 \rightarrow Y \rightarrow P \rightarrow X \rightarrow 0$ in $\mathcal{S}$ such that $P$ is projective.

Recall that these notions permit the definition of extension functors $\operatorname{Ext}_{\mathcal{E}}^{i}$ which satisfy the expected properties, either from Yoneda's structure of long exact sequences, or using projective resolutions if $\mathcal{E}$ has enough projective objects, or using injective resolutions if $\mathcal{E}$ has enough injective objects, or more generally using the derived category of $\mathcal{E}$.

Throughout this paper, we will use the following definition:

Definition 3.2. Let $\mathcal{E}$ and $\mathcal{E}^{\prime}$ be exact categories and $F: \mathcal{E} \rightarrow \mathcal{E}^{\prime}$ an exact functor. We say that $F$ is exact bijective if the induced morphism $\operatorname{Ext}_{\mathcal{E}}^{1}(-,-) \rightarrow \operatorname{Ext}_{\mathcal{E}^{\prime}}^{1}(F-, F-)$ is an isomorphism. We say that $F$ is an equivalence of exact categories if it is an exact bijective equivalence of categories (or, equivalently, an exact equivalence of categories with an exact quasi-inverse).

A typical example of exact bijective functor arises when $\mathcal{E}$ is a full exact subcategory of $\mathcal{E}^{\prime}$ (i.e., a full subcategory which is closed under extensions).

Remark 3.3. Assume $F: \mathcal{E} \rightarrow \mathcal{E}^{\prime}$ is a dense and exact bijective functor. Then:

(a) For any $X \in \mathcal{E}$, we have $X$ is projective if and only if $F X$ is projective, and the dual statement holds for injectivity. 
(b) $\mathcal{E}$ has enough projective objects if and only if $\mathcal{E}^{\prime}$ has enough projective objects, and the dual statement holds for injectivity.

(c) $\mathcal{E}$ is Frobenius if and only if $\mathcal{E}^{\prime}$ is Frobenius.

We give an elementary result about second extension groups:

Proposition 3.4. Let $F: \mathcal{E} \rightarrow \mathcal{E}^{\prime}$ be an exact bijective functor. Then, it induces a canonical natural monomorphism $\operatorname{Ext}_{\mathcal{E}}^{2}(-,-) \hookrightarrow \operatorname{Ext}_{\mathcal{E}^{\prime}}^{2}(F-, F-)$.

Proof. The existence of a map $\varphi: \operatorname{Ext}_{\mathcal{E}}^{2}(-,-) \rightarrow \operatorname{Ext}_{\mathcal{E}^{\prime}}^{2}(F-, F-)$ is immediate. We consider an admissible 4-term exact sequence $\xi: 0 \rightarrow X \rightarrow Y_{1} \rightarrow Y_{2} \rightarrow Z \rightarrow 0$ which, by definition, comes from two short exact sequences

$$
\xi_{1}: 0 \rightarrow X \rightarrow Y_{1} \rightarrow Y \rightarrow 0 \quad \text { and } \quad \xi_{2}: 0 \rightarrow Y \rightarrow Y_{2} \stackrel{u}{\rightarrow} Z \rightarrow 0 .
$$

Suppose that $\xi \in \operatorname{Ker} \varphi_{Z, X}$. Applying $\operatorname{Hom}_{\mathcal{E}}(-, X)$ and $\operatorname{Hom}_{\mathcal{E}^{\prime}}(F-, F X)$ to $\xi_{2}$ gives a commutative diagram of exact sequences:

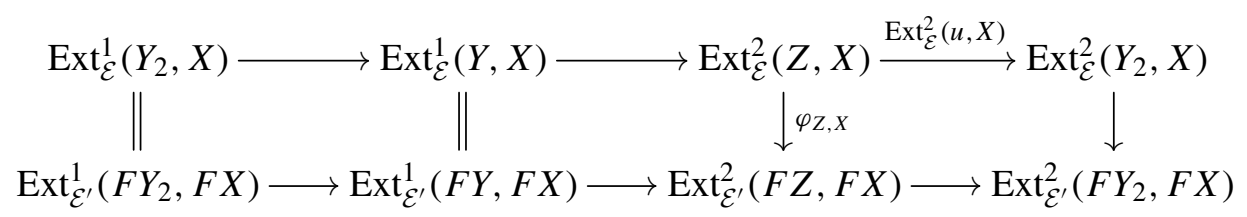

By the definition of Yoneda product, $\xi \in \operatorname{Ker}_{\operatorname{Ext}_{\mathcal{E}}^{2}}^{2}(u, X)$, so an easy diagram chase gives $\xi=0$.

Let us define important concepts:

Definition 3.5. Let $\mathcal{E}$ be a Krull-Schmidt additive category and $\mathcal{E}^{\prime} \subset \mathcal{E}$ an additive subcategory. Then:

(a) We say that $f: X \rightarrow Y$ in $\mathcal{E}$ is left minimal if for any $g \in \operatorname{End}_{\mathcal{E}}(Y)$ such that $f g=f$, the map $g$ is invertible, or equivalently, if for any idempotent $e \in \operatorname{End}_{\mathcal{E}}(Y)$, we have $f e=f$ implies $e=\operatorname{id}_{Y}$.

(b) We say that $g: Y \rightarrow X$ in $\mathcal{E}$ is right minimal if for any $f \in \operatorname{End}_{\mathcal{E}}(Y)$ such that $f g=g$, the map $f$ is invertible, or equivalently, if for any idempotent $e \in \operatorname{End}_{\mathcal{E}}(Y)$, we have $e g=g$ implies $e=\operatorname{id}_{Y}$.

(c) We say that $f: X \rightarrow X^{\prime}$ in $\mathcal{E}$ is a left $\mathcal{E}^{\prime}$-approximation (of $X$ ) if $X^{\prime} \in \mathcal{E}^{\prime}$ and any morphism from $X$ to any object of $\mathcal{E}^{\prime}$ factors through $f$.

(d) We say that $g: X^{\prime} \rightarrow X$ in $\mathcal{E}$ is a right $\mathcal{E}^{\prime}$-approximation (of $X$ ) if $X^{\prime} \in \mathcal{E}^{\prime}$ and any morphism from any object of $\mathcal{E}^{\prime}$ to $X$ factors through $g$.

Notice that, in the situation of the previous definition, if an object $X \in \mathcal{E}$ admits a left $\mathcal{E}^{\prime}$-approximation, then it admits a left minimal $\mathcal{E}^{\prime}$-approximation which is unique up to isomorphism, and an analogous statement holds for right $\mathcal{E}^{\prime}$-approximations. 
3B. Exact ideal quotients of an exact category. Let $(\mathcal{E}, \mathcal{S})$ be an exact category and $\mathcal{E}^{\prime}$ a full subcategory of $\mathcal{E}$ which is closed under extensions. Then $\left(\mathcal{E}^{\prime}, \mathcal{S}^{\prime}\right)$ forms an exact category for the family $\mathcal{S}^{\prime}$ of all admissible exact sequences in $\mathcal{S}$ whose terms belong to $\mathcal{E}^{\prime}$.

We denote by $\mathcal{F}$ a subcategory of $\mathcal{E}$ satisfying $\operatorname{Ext}_{\mathcal{E}}^{1}\left(\mathcal{F}, \mathcal{E}^{\prime}\right)=\operatorname{Ext}_{\mathcal{E}}^{1}\left(\mathcal{E}^{\prime}, \mathcal{F}\right)=0$. Let $\mathcal{S}_{\mathcal{F}}^{\prime}$ be the class of pairs of morphisms in $\mathcal{E}^{\prime} /[\mathcal{F}]$ which are isomorphic to a pair in $\pi\left(\mathcal{S}^{\prime}\right)$, where $\pi: \mathcal{E}^{\prime} \rightarrow \mathcal{E}^{\prime} /[\mathcal{F}]$ is the canonical functor.

Theorem 3.6. The following are equivalent:

(i) $\left(\mathcal{E}^{\prime} /[\mathcal{F}], \mathcal{S}_{\mathcal{F}}^{\prime}\right)$ is exact.

(ii) For any admissible monomorphism $f$ of $\left(\mathcal{E}^{\prime}, \mathcal{S}^{\prime}\right)$, the map $\pi(f)$ is a categorical monomorphism in $\mathcal{E}^{\prime} /[\mathcal{F}]$, and the dual statement holds for epimorphisms.

In this case, $\pi: \mathcal{E}^{\prime} \rightarrow \mathcal{E}^{\prime} /[\mathcal{F}]$ is automatically exact bijective.

Proof. (i) $\Rightarrow$ (ii) is trivial. Let us prove the converse. Let us first check that any $(\bar{f}, \bar{g}) \in \mathcal{S}_{\mathcal{F}}^{\prime}$ is a kernel-cokernel pair. By (ii), $\bar{f}$ is a monomorphism and $\bar{g}$ is an epimorphism. By definition, we can lift $(\bar{f}, \bar{g})$ to $(f, g) \in \mathcal{S}^{\prime}$. Suppose that $\bar{f} \bar{h}=0$ for some morphism $\bar{h}$ of $\mathcal{E}^{\prime} /[\mathcal{F}]$. By definition, it means that there is a commutative diagram in $\mathcal{E}$ of the form

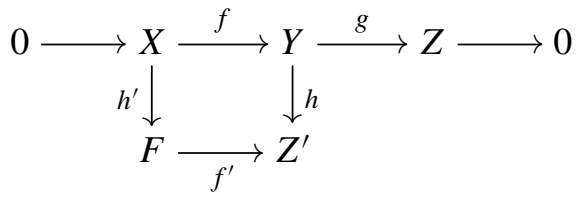

with $F \in \mathcal{F}$. As $\operatorname{Ext}_{\mathcal{E}}^{1}(Z, F)=0$, there exists $u: Y \rightarrow F$ such that $h^{\prime}=f u$. Thus, $h=u f^{\prime}+g v$ for some $v: Z \rightarrow Z^{\prime}$ and $\bar{h}=\bar{g} \bar{v}$ holds. This proves that $\bar{g}$ is a cokernel of $\bar{f}$. Dually, we prove that $\bar{f}$ is a kernel of $\bar{g}$.

Let us check axioms of exact categories one by one: (Ex0): This is obvious.

(Ex1): Suppose that $\bar{g}: X \rightarrow X^{\prime}$ and $\bar{g}^{\prime}: X^{\prime} \rightarrow X^{\prime \prime}$ are epimorphisms in $\mathcal{S}_{\mathcal{F}}^{\prime}$. It is easy to check that we can lift them to admissible epimorphisms $g: X \oplus F_{1} \rightarrow X^{\prime} \oplus F_{2}$ and $g^{\prime}: X^{\prime} \oplus F_{3} \rightarrow X^{\prime \prime} \oplus F_{4}$ of $\mathcal{E}^{\prime}$. Thus $\bar{g} \bar{g}^{\prime}$ can be lifted to an admissible epimorphism $X \oplus F_{1} \oplus F_{3} \rightarrow X^{\prime \prime} \oplus F_{2} \oplus F_{4}$ in $\mathcal{E}^{\prime}$ using (Ex1) in $\left(\mathcal{E}^{\prime}, \mathcal{S}^{\prime}\right)$. By definition, $\bar{g} \bar{g}^{\prime}$ is then an epimorphism in $\mathcal{S}_{\mathcal{F}}^{\prime}$.

$(\operatorname{Ex} 1)^{\mathrm{op}}$ : This is the dual of the previous item.

(Ex2): Let $\bar{g}: Y \rightarrow Z$ be an epimorphism in $\mathcal{S}_{\mathcal{F}}^{\prime}$ and $\bar{v}: Z^{\prime} \rightarrow Z$ be a morphism in $\mathcal{E}^{\prime} /[\mathcal{F}]$. Without loss of generality, we can suppose that they come from lifts $g: Y \rightarrow Z$ and $v: Z^{\prime} \rightarrow Z$ in $\mathcal{E}^{\prime}$, where $g$ is an admissible epimorphism. Thus, we 
can complete the pair to a pullback diagram

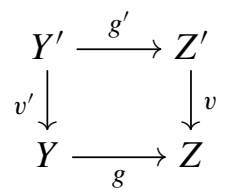

in $\mathcal{E}^{\prime}$, where $g^{\prime}$ is an admissible epimorphism. Then $0 \rightarrow Y^{\prime} \rightarrow Z^{\prime} \oplus Y \rightarrow Z \rightarrow 0$ is in $\mathcal{S}^{\prime}$, and its projection to $\mathcal{E}^{\prime} /[\mathcal{F}]$ is in $\mathcal{S}_{\mathcal{F}}^{\prime}$. Thus the diagram is also a pullback diagram in $\mathcal{E}^{\prime} /[\mathcal{F}]$, and $\bar{g}^{\prime}$ is an epimorphism in $\mathcal{S}_{\mathcal{F}}^{\prime}$.

$(\mathrm{Ex} 2)^{\mathrm{op}}$ : This is the dual of the previous item.

We have finished proving the equivalence. Let us check that the projection $\pi: \mathcal{E}^{\prime} \rightarrow \mathcal{E}^{\prime} /[\mathcal{F}]$ is exact bijective. First of all, for $X, Z \in \mathcal{E}^{\prime}$, the induced map $\operatorname{Ext}_{\mathcal{E}^{\prime}}^{1}(Z, X) \rightarrow \operatorname{Ext}_{\mathcal{E}^{\prime} /[\mathcal{F}]}^{1}(\pi Z, \pi X)$ is clearly surjective. To prove that it is injective, let us consider a short exact sequence

$$
0 \rightarrow X \stackrel{f}{\longrightarrow} Y \stackrel{g}{\longrightarrow} Z \rightarrow 0,
$$

which splits in $\mathcal{E}^{\prime} /[\mathcal{F}]$. By definition, it means that there is $g^{\prime}: Z \rightarrow Y$ and two morphisms $u: Z \rightarrow F$ and $v: F \rightarrow Z$ with $F \in \mathcal{F}$ such that $\operatorname{id}_{Z}=g^{\prime} g+u v$. As $\operatorname{Ext}_{\mathcal{E}}^{1}(F, X)=0$, there exists $v^{\prime}: F \rightarrow Y$ such that $v=v^{\prime} g$. Thus $\operatorname{id}_{Z}=\left(g^{\prime}+\right.$ $\left.u v^{\prime}\right) g$ holds, and (3-1) splits in $\mathcal{E}^{\prime}$. Therefore $\operatorname{Ext}_{\mathcal{E}^{\prime}}^{1}(Z, X) \rightarrow \operatorname{Ext}_{\mathcal{E}^{\prime} /[\mathcal{F}]}^{1}(\pi Z, \pi X)$ is injective.

In the rest of this section we give sufficient conditions for Theorem 3.6(ii) to hold. For two subcategories $\mathcal{B}$ and $\mathcal{C}$ of $\mathcal{E}$, we denote by $\mathcal{C} \searrow \mathcal{B}$ the full subcategory of $\mathcal{E}$ consisting of $X$ such that for any complex $Y \stackrel{g}{\longrightarrow} B \stackrel{f}{\longrightarrow} X$ with $B \in \mathcal{B}$ and $Y \in \mathcal{E}^{\prime}$, there exists a morphism of complexes

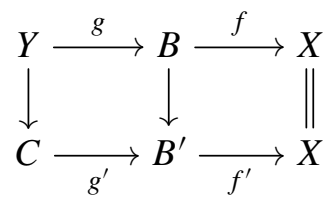

with $B^{\prime} \in \mathcal{B}$ and $C \in \mathcal{C}$. Notice that, if $X \in \mathcal{E}$ has a right $\mathcal{B}$-approximation whose pseudo-kernel is in $\mathcal{C}$, then $X \in[\mathcal{C} \searrow \mathcal{B}]$. Also notice that $\left[\mathcal{E}^{\prime} \searrow \mathcal{B}\right]=\mathcal{E}$ holds since we can choose $f^{\prime}=f$ and $g^{\prime}=g$. Dually, we denote by $\mathcal{B} \nearrow \mathcal{C}$ the full subcategory of $\mathcal{E}$ consisting of $X$ such that for any complex $X \stackrel{f}{\longrightarrow} B \stackrel{g}{\longrightarrow} Y$ with $B \in \mathcal{B}$ and $Y \in \mathcal{E}^{\prime}$, there exists a morphism of complexes

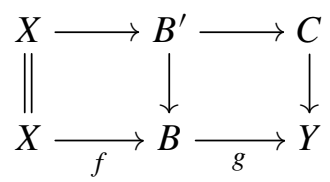


with $B^{\prime} \in \mathcal{B}$ and $C \in \mathcal{C}$. As before, if $X \in \mathcal{E}$ has a left $\mathcal{B}$-approximation whose pseudo-cokernel is in $\mathcal{C}$, then $X \in[\mathcal{B} \nearrow \mathcal{C}]$. Also, we get $\left[\mathcal{B} \nearrow \mathcal{E}^{\prime}\right]=\mathcal{E}$. We get the following corollary:

Corollary 3.7. Let $\mathcal{P}$ and $\mathcal{I}$ be the full subcategories of $\mathcal{E}$ consisting of objects $X$ satisfying $\operatorname{Ext}_{\mathcal{E}}^{1}\left(X, \mathcal{E}^{\prime}\right)=0$ and $\operatorname{Ext}_{\mathcal{E}}^{1}\left(\mathcal{E}^{\prime}, X\right)=0$ respectively. If

$$
\mathcal{E}^{\prime} \subset(\mathcal{F} \nearrow[\mathcal{I} \searrow \mathcal{F}]) \cap([\mathcal{F} \nearrow \mathcal{P}] \searrow \mathcal{F})
$$

then $\left(\mathcal{E}^{\prime} /[\mathcal{F}], \mathcal{S}_{\mathcal{F}}^{\prime}\right)$ is an exact category.

Proof. We need to prove Theorem 3.6(ii). We do it for admissible monomorphisms. Let $0 \rightarrow X \stackrel{f}{\longrightarrow} Y \stackrel{g}{\longrightarrow} Z \rightarrow 0$ be a short exact sequence in $\mathcal{S}^{\prime}$, and let $u: X^{\prime} \rightarrow X$ be a morphism such that $\bar{u} \bar{f}=0$ in $\mathcal{E} /[\mathcal{F}]$. Then $u f=f^{\prime} u^{\prime}$ holds for some $f^{\prime}: X^{\prime} \rightarrow F^{\prime}$ and $u^{\prime}: F^{\prime} \rightarrow Y$ with $F^{\prime} \in \mathcal{F}$.

Suppose first that $X^{\prime} \in[\mathcal{F} \nearrow \mathcal{P}]$. By definition, we can complete a commutative diagram

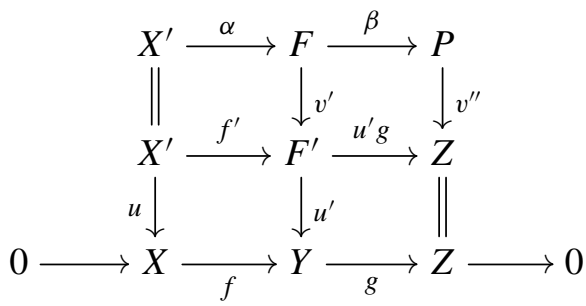

with $\alpha \beta=0$ and $F \in \mathcal{F}$ and $P \in \mathcal{P}$. As $\operatorname{Ext}_{\mathcal{E}}^{1}(P, X)=0$, we know $v^{\prime \prime}=g^{\prime \prime} g$ for some $g^{\prime \prime}: P \rightarrow Y$ and we easily get $v^{\prime} u^{\prime}=\beta g^{\prime \prime}+f^{\prime \prime} f$ for some $f^{\prime \prime}: F \rightarrow X$. We deduce that $\alpha f^{\prime \prime} f=\alpha v^{\prime} u^{\prime}-\alpha \beta g^{\prime \prime}=u f$. As $f$ is a monomorphism, $\alpha f^{\prime \prime}=u$ and therefore $\bar{u}=0$.

Let us now suppose that $X^{\prime} \in \mathcal{E}^{\prime}$. As $Z \in([\mathcal{F} \nearrow \mathcal{P}] \searrow \mathcal{F})$, we can complete the following commutative diagram

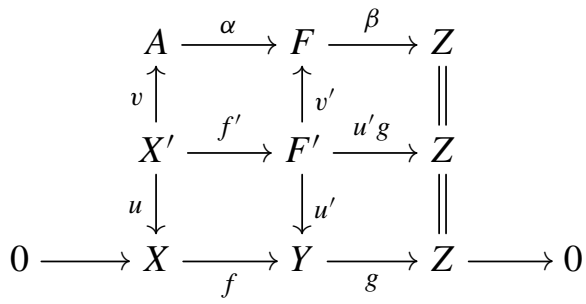

with $\alpha \beta=0$ and $F \in \mathcal{F}$ and $A \in[\mathcal{F} \nearrow \mathcal{P}]$. Then, $\operatorname{asxt}_{\mathcal{E}}^{1}(F, X)=0$, we get $\beta=\beta^{\prime} g$ with $\beta^{\prime}: F \rightarrow Y$ and, as $f$ is the kernel of $g$, there exists $\alpha^{\prime}: A \rightarrow X$ such that $\alpha \beta^{\prime}=$ $\alpha^{\prime} f$. As $A \in[\mathcal{F} \nearrow \mathcal{P}]$ and $\bar{\alpha}^{\prime} \bar{f}=0$, by the first part of the argument, $\bar{\alpha}^{\prime}=0$. On the other hand, by an easy diagram chase, there exists $w: F^{\prime} \rightarrow X$ such that $u^{\prime}=v^{\prime} \beta^{\prime}+$ $w f$. So we get $u f=f^{\prime} u^{\prime}=f^{\prime} v^{\prime} \beta^{\prime}+f^{\prime} w f=v \alpha \beta^{\prime}+f^{\prime} w f=v \alpha^{\prime} f+f^{\prime} w f$. As $f$ is a monomorphism, we deduce that $u=v \alpha^{\prime}+f^{\prime} w$. Thus $\bar{u}=0$ holds since $\bar{\alpha}^{\prime}=0$. 
In the rest of this section, we give three special cases as an application. Notice that the first case recovers Chen's result [2012, Theorem 3.1] for $\mathcal{E}^{\prime}=\mathcal{E}$.

Corollary 3.8. (a) If, for any $X \in \mathcal{E}^{\prime}$, there exist left and right $\mathcal{F}$-approximations $f$ and $f^{\prime}$ and pseudo-cokernel $g$ and pseudo-kernel $g^{\prime}$

$$
X \stackrel{f}{\longrightarrow} F^{X} \stackrel{g}{\longrightarrow} P^{X} \text { and } I_{X} \stackrel{g^{\prime}}{\longrightarrow} F_{X} \stackrel{f^{\prime}}{\longrightarrow} X
$$

such that $P^{X} \in \mathcal{P}$ and $I_{X} \in \mathcal{I}$ then $\left(\mathcal{E}^{\prime} /[\mathcal{F}], \mathcal{S}_{\mathcal{F}}^{\prime}\right)$ is an exact category.

(b) If, for any $X \in \mathcal{E}^{\prime}$, there exists a left $\mathcal{F}$-approximation $X \rightarrow F^{X}$ which is a categorical epimorphism, then $\left(\mathcal{E}^{\prime} /[\mathcal{F}], \mathcal{S}_{\mathcal{F}}^{\prime}\right)$ is an exact category.

(c) If, for any $X \in \mathcal{E}^{\prime}$, there exists a right $\mathcal{F}$-approximation $F_{X} \rightarrow X$ which is a categorical monomorphism, then $\left(\mathcal{E}^{\prime} /[\mathcal{F}], \mathcal{S}_{\mathcal{F}}^{\prime}\right)$ is an exact category.

Proof. (a) Let $X \rightarrow F \rightarrow Y$ be a complex where $X, Y \in \mathcal{E}^{\prime}$ and $F \in \mathcal{F}$. It is easy to complete the following commutative diagram

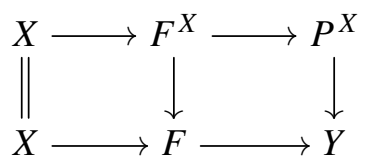

so $\mathcal{E}^{\prime} \subset[\mathcal{F} \nearrow \mathcal{P}]$. Thus we have $\mathcal{E}=\left[\mathcal{E}^{\prime} \searrow \mathcal{F}\right] \subset([\mathcal{F} \nearrow \mathcal{P}] \searrow \mathcal{F})$. Dually we have $\mathcal{E}=(\mathcal{F} \nearrow[\mathcal{I} \searrow \mathcal{F}])$.

(b) By the same argument as the beginning of (a), we get $\mathcal{E}^{\prime} \subset[\mathcal{F} \nearrow 0]$. So $\mathcal{E}^{\prime} \subset[\mathcal{F} \nearrow 0] \subset(\mathcal{F} \nearrow[\mathcal{I} \searrow \mathcal{F}])$ and $\mathcal{E}=\left[\mathcal{E}^{\prime} \searrow \mathcal{F}\right] \subset([\mathcal{F} \nearrow 0] \searrow \mathcal{F}) \subset([\mathcal{F} \nearrow \mathcal{P}] \searrow \mathcal{F})$.

(c) This is the dual of (b).

3C. On some Frobenius subcategories of exact categories. When we have an admissible monomorphism $f: X \rightarrow Y$ in an exact category, we say $X$ is an admissible subobject of $Y$. Dually we define an admissible factor object. For a full subcategory $\mathcal{E}^{\prime}$ of an exact category $\mathcal{E}$, we denote by $\operatorname{Sub} \mathcal{E}^{\prime}$ the smallest full subcategory of $\mathcal{E}$ which is closed under admissible subobjects and contains add $\mathcal{E}^{\prime}$.

We recall that an exact category is Frobenius if it has enough injective objects, enough projective objects and they coincide. This subsection is devoted to proving the following result.

Proposition 3.9. Let $\mathcal{E}$ be an exact category which has enough projective objects and enough injective objects. Let $\mathcal{U}$ be a subcategory of injective objects in $\mathcal{E}$ satisfying $\mathcal{U}=\operatorname{add} \mathcal{U}$, and let $\mathcal{D}:=\operatorname{Sub} \mathcal{U}$. Assume that projective objects of $\mathcal{E}$ and those of $\mathcal{D}$ coincide. Then the following assertions hold:

(a) $\mathcal{D}$ is closed under extensions. 
(b) $\mathcal{D}$ is Frobenius if and only if the following conditions are satisfied:

- $U$ is projective-injective in $\mathcal{E}$ for any $U \in \mathcal{U}$.

- Each projective object of $\mathcal{E}$ has injective dimension at most 1 and each injective object of $\mathcal{E}$ has projective dimension at most 1 .

(c) If the conditions in $(b)$ are satisfied, then $\mathcal{U}$ is the category of projectiveinjective objects in $\mathcal{E}$.

Part (a) is an easy consequence of the horseshoe lemma. Let us start with the following lemma:

Lemma 3.10. Assume any object in $\mathcal{U}$ is projective in $\mathcal{E}$. Let $0 \rightarrow E \rightarrow E^{\prime} \stackrel{f}{\longrightarrow} I \rightarrow 0$ be an exact sequence in $\mathcal{E}$ with I injective. Then

$$
\operatorname{Hom}_{\mathcal{E}}(\mathcal{D}, f): \operatorname{Hom}_{\mathcal{E}}\left(\mathcal{D}, E^{\prime}\right) \rightarrow \operatorname{Hom}_{\mathcal{E}}(\mathcal{D}, I)
$$

is an epimorphism.

Proof. Take a morphism $g: D \rightarrow I$ with $D \in \mathcal{D}$. Then there exists an admissible monomorphism $i: D \rightarrow U$ with $U \in \mathcal{U}$. Since $I$ is injective in $\mathcal{E}$, there exists $s: U \rightarrow I$ such that $g=i s$. Since $U$ is projective in $\mathcal{E}$, there exists $t: U \rightarrow E^{\prime}$ such that $s=t f$ :

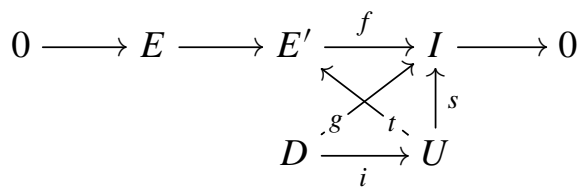

Since $g=i t f$, we have the assertion.

Let us now prove the proposition.

Proof of Proposition 3.9(b). " $\Rightarrow$ " Suppose that $\mathcal{D}$ is Frobenius. Note that our assumptions imply that projective objects in $\mathcal{E}$, projective objects in $\mathcal{D}$ and injective objects in $\mathcal{D}$ coincide.

Fix any $U \in \mathcal{U}$. Then $U$ is injective in $\mathcal{E}$ by our assumption, and hence $U$ is injective also in $\mathcal{D}$. Therefore $U$ is projective in $\mathcal{E}$ by the remark above.

Let $P$ be a projective object in $\mathcal{E}$. Then $P$ is projective-injective in $\mathcal{D}$. Since our assumptions imply $\Omega_{\mathcal{E}}(\mathcal{E}) \subset \mathcal{D}$, we have

$$
\operatorname{Ext}_{\mathcal{E}}^{2}(\mathcal{E}, P)=\operatorname{Ext}_{\mathcal{E}}^{1}\left(\Omega_{\mathcal{E}}(\mathcal{E}), P\right)=0 .
$$

Thus $P$ has injective dimension at most 1 in $\mathcal{E}$.

Let $I$ be an injective object in $\mathcal{E}$. We take an exact sequence

$$
0 \rightarrow \Omega_{\mathcal{E}}(I) \rightarrow P \stackrel{f}{\longrightarrow} I \rightarrow 0
$$


with a projective object $P$ in $\mathcal{E}$. Our assumptions imply $P \in \mathcal{D}$ and $\Omega_{\mathcal{E}}(I) \in \mathcal{D}$. We apply $\operatorname{Hom}_{\mathcal{E}}(\mathcal{D},-)$ to (3-2) to get the exact sequence

$$
\operatorname{Hom}_{\mathcal{E}}(\mathcal{D}, P) \rightarrow \operatorname{Hom}_{\mathcal{E}}(\mathcal{D}, I) \rightarrow \operatorname{Ext}_{\mathcal{E}}^{1}\left(\mathcal{D}, \Omega_{\mathcal{E}}(I)\right) \rightarrow \operatorname{Ext}_{\mathcal{E}}^{1}(\mathcal{D}, P)=0 .
$$

By Lemma 3.10, we have $\operatorname{Ext}_{\mathcal{E}}^{1}\left(\mathcal{D}, \Omega_{\mathcal{E}}(I)\right)=0$. Thus $\Omega_{\mathcal{E}}(I)$ is projective-injective in $\mathcal{D}$ so projective in $\mathcal{E}$, and the assertion follows.

" $\Leftarrow$ " Let $P$ be a projective object in $\mathcal{D}$. By our assumptions, $P$ is projective in $\mathcal{E}$, and there exists an exact sequence $0 \rightarrow P \rightarrow I^{0} \rightarrow I^{1} \rightarrow 0$ with injective objects $I^{0}, I^{1}$ in $\mathcal{E}$. Applying $\operatorname{Hom}_{\mathcal{E}}(\mathcal{D},-)$, we have an exact sequence

$$
\operatorname{Hom}_{\mathcal{E}}\left(\mathcal{D}, I^{0}\right) \rightarrow \operatorname{Hom}_{\mathcal{E}}\left(\mathcal{D}, I^{1}\right) \rightarrow \operatorname{Ext}_{\mathcal{E}}^{1}(\mathcal{D}, P) \rightarrow \operatorname{Ext}_{\mathcal{E}}^{1}\left(\mathcal{D}, I^{0}\right)=0 .
$$

By Lemma 3.10, we have $\operatorname{Ext}_{\mathcal{E}}^{1}(\mathcal{D}, P)=0$. Thus $P$ is injective in $\mathcal{D}$.

Let $I$ be an injective object in $\mathcal{D}$. Since $\Omega_{\mathcal{E}}(\mathcal{E}) \subset \mathcal{D}$, we have $\operatorname{Ext}_{\mathcal{E}}^{2}(\mathcal{E}, I)=$ $\operatorname{Ext}_{\mathcal{E}}^{1}\left(\Omega_{\mathcal{E}}(\mathcal{E}), I\right)=0$. Thus $I$ has injective dimension at most 1 in $\mathcal{E}$. Now we take an exact sequence

$$
0 \rightarrow I \rightarrow U \rightarrow E \rightarrow 0
$$

with $U \in \mathcal{U}$ and $E \in \mathcal{E}$. Since $U$ is injective in $\mathcal{E}$, so is $E$. Thus $E$ has projective dimension at most 1 in $\mathcal{E}$. Since $U$ is projective in $\mathcal{E}$, so is $I$. Thus $I$ is projective in $\mathcal{D}$.

Since $\mathcal{E}$ has enough projective objects and $\Omega_{\mathcal{E}}(\mathcal{E}) \subset \mathcal{D}$ holds, $\mathcal{D}$ also has enough projective objects. It remains to prove that $\mathcal{D}$ has enough injective objects. Fix $D \in \mathcal{D}$ and take an exact sequence $0 \rightarrow D \rightarrow U \rightarrow E \rightarrow 0$ with $U \in \mathcal{U}$ and $E \in \mathcal{E}$. Since $\mathcal{E}$ has enough injective objects by our assumption, there exists an exact sequence $0 \rightarrow E \rightarrow I \rightarrow E^{\prime} \rightarrow 0$ with an injective object $I$ in $\mathcal{E}$ and $E^{\prime} \in \mathcal{E}$. Let $0 \rightarrow P_{1} \rightarrow P_{0} \rightarrow I \rightarrow 0$ be a projective resolution of $I$ in $\mathcal{E}$. We have a commutative diagram of exact sequences:

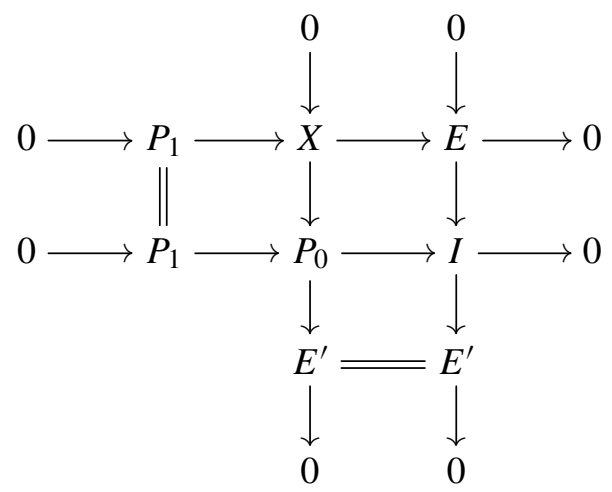


Since $P_{0} \in \mathcal{D}$, the middle column shows $X \in \mathcal{D}$. On the other hand, we have the following commutative diagram of exact sequences:

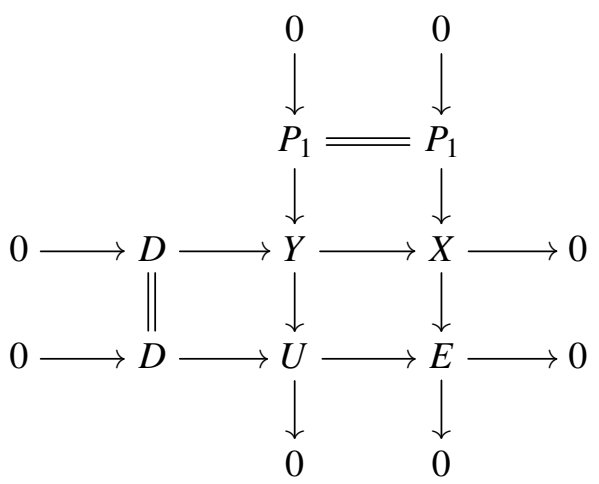

As $P_{1}$ is projective-injective in $\mathcal{D}$, the middle column splits and $Y \cong U \oplus P_{1}$ is injective in $\mathcal{D}$. The middle row gives an injective hull of $D$ in $\mathcal{D}$.

Proof of Proposition 3.9(c). Let $P$ be a projective-injective object in $\mathcal{E}$. Then it belongs to $\mathcal{D}$, and there is a short exact sequence $0 \rightarrow P \rightarrow U \rightarrow E \rightarrow 0$ with $U \in \mathcal{U}$ and $E \in \mathcal{E}$. Since $P$ is injective in $\mathcal{E}$, this sequence splits. Thus $P$ belongs to $\mathcal{U}$.

\section{Equivalences arising from torsion pairs on exact categories}

Throughout this section, we assume the following:

- $\mathcal{E}$ is an exact category which is Krull-Schmidt.

- $(\mathcal{A}, \mathcal{B})$ is a torsion pair of $\mathcal{E}$; that is, the following conditions are satisfied:

- $\mathcal{A}$ and $\mathcal{B}$ are full subcategories of $\mathcal{E}$ such that $\operatorname{Hom}_{\mathcal{E}}(\mathcal{A}, \mathcal{B})=0$.

- For any $E \in \mathcal{E}$, there exists an exact sequence $0 \rightarrow A \rightarrow E \rightarrow B \rightarrow 0$ with $A \in \mathcal{A}$ and $B \in \mathcal{B}$.

Then $\mathcal{A}$ is closed under taking extensions and admissible factor objects, and $\mathcal{B}$ is closed under taking extensions and admissible subobjects. On the other hand, the natural inclusion functor $\mathcal{B} \rightarrow \mathcal{E}$ has a left adjoint functor $F: \mathcal{E} \rightarrow \mathcal{B}$. This is dense and induces a dense functor

$$
F: \mathcal{E} /[\mathcal{A}] \rightarrow \mathcal{B}
$$

4A. Basic properties of $\boldsymbol{F}: \mathcal{E} /[\mathcal{A}] \rightarrow \mathcal{B}$. We consider the full subcategories of $\mathcal{E}$ defined by

$$
\begin{aligned}
& \mathcal{E}_{1}=\left\{X \in \mathcal{E} \mid \operatorname{Ext}_{\mathcal{E}}^{1}(X, \mathcal{A})=0\right\}, \\
& \mathcal{E}_{2}=\left\{X \in \mathcal{E} \mid \operatorname{Ext}_{\mathcal{E}}^{1}(X, \mathcal{A})=0, \operatorname{Ext}_{\mathcal{E}}^{2}(X, \mathcal{A})=0\right\} .
\end{aligned}
$$

The subsection is devoted to proving the following result: 
Theorem 4.1. We have the following assertions:

(a) The functor $F: \mathcal{E}_{1} /[\mathcal{A}] \rightarrow \mathcal{B}$ is fully faithful.

(b) The essential image of $F: \mathcal{E}_{1} /[\mathcal{A}] \rightarrow \mathcal{B}$ is the subcategory consisting of $B \in \mathcal{B}$ such that $\operatorname{Ext}_{\mathcal{E}}^{1}(B, \mathcal{A})$ is a finitely generated $\mathcal{A}^{\mathrm{op}}$-module.

(c) If $\operatorname{Ext}_{\mathcal{E}}^{1}(\mathcal{A}, \mathcal{B})=0$, then $F: \mathcal{E}_{2} \rightarrow \mathcal{B}$ is exact bijective.

(d) If any object in $\mathcal{A}$ is projective in $\mathcal{E}$, then $\mathcal{E}_{2} /[\mathcal{A}]$ inherits canonically the exact structure of $\mathcal{E}_{2}$ and $F: \mathcal{E}_{2} /[\mathcal{A}] \rightarrow \mathcal{B}$ is exact bijective.

We denote by $T: \mathcal{E} \rightarrow \mathcal{A}$ the right adjoint functor of the inclusion functor $\mathcal{A} \rightarrow \mathcal{E}$. Then for any $E \in \mathcal{E}$, there exists a short exact sequence

$$
0 \rightarrow T E \stackrel{f}{\longrightarrow} E \stackrel{g}{\longrightarrow} F E \rightarrow 0
$$

in $\mathcal{E}$ with $T E \in \mathcal{A}$ and $F E \in \mathcal{B}$. Clearly $f$ is a right $\mathcal{A}$-approximation and $g$ is a left $\mathcal{B}$-approximation.

The proof of Theorem 4.1 is divided into Lemmas 4.2, 4.3, 4.5 and 4.6.

Lemma 4.2. The functor $F: \mathcal{E}_{1} \rightarrow \mathcal{B}$ induces a fully faithful functor $F: \mathcal{E}_{1} /[\mathcal{A}] \rightarrow \mathcal{B}$.

Proof. Fix $X, Y \in \mathcal{E}_{1}$. By applying $\operatorname{Hom}_{\mathcal{E}}(X,-)$ to the short exact sequence $0 \rightarrow T Y \rightarrow Y \rightarrow F Y \rightarrow 0$, we obtain the short exact sequence

$$
0 \rightarrow \operatorname{Hom}_{\mathcal{E}}(X, T Y) \rightarrow \operatorname{Hom}_{\mathcal{E}}(X, Y) \rightarrow \operatorname{Hom}_{\mathcal{E}}(X, F Y) \rightarrow \operatorname{Ext}_{\mathcal{E}}^{1}(X, T Y)=0,
$$

where the last equality follows from $X \in \mathcal{E}_{1}$. So

$$
\operatorname{Hom}_{\mathcal{E}}(X, F Y) \cong \frac{\operatorname{Hom}_{\mathcal{E}}(X, Y)}{\operatorname{Hom}_{\mathcal{E}}(X, T Y)}=\operatorname{Hom}_{\mathcal{E} /[\mathcal{A}]}(X, Y),
$$

where we use the fact that the first arrow of $T Y \rightarrow Y$ is a right $\mathcal{A}$-approximation. On the other hand, using adjunction we have an isomorphism

$$
\operatorname{Hom}_{\mathcal{E}}(F X, F Y) \cong \operatorname{Hom}_{\mathcal{E}}(X, F Y) .
$$

Thus the assertion follows.

Next we prove the following observation.

Proposition 4.3. The following conditions are equivalent for $B \in \mathcal{B}$ :

(i) $B$ belongs to the essential image of $F: \mathcal{E}_{1} \rightarrow \mathcal{B}$.

(ii) $\operatorname{Ext}_{\mathcal{E}}^{1}(B, \mathcal{A})$ is a finitely generated $\mathcal{A}^{\mathrm{op}}$-module.

This follows immediately from the following result for Krull-Schmidt exact categories, which is a generalization of [Auslander and Reiten 1991, Proposition 1.4].

Lemma 4.4. Let $\mathcal{X}$ be a Krull-Schmidt exact category, and $\mathcal{Y}$ a subcategory of $\mathcal{X}$ which is closed under extensions and direct summands. For $X \in \mathcal{X}$, the following conditions are equivalent: 
(i) There exists an exact sequence $0 \rightarrow Y \rightarrow Z \rightarrow X \rightarrow 0$ with $Y \in \mathcal{Y}$ and $\operatorname{Ext}_{\mathcal{X}}^{1}(Z, \mathcal{Y})=0$

(ii) $\operatorname{Ext}_{\mathcal{X}}^{1}(X, \mathcal{Y})$ is finitely generated $\mathcal{Y}^{\mathrm{op}}$-module.

We include a proof for the convenience of the reader.

Proof. (i) $\Rightarrow$ (ii): Applying $\operatorname{Hom}_{\mathcal{X}}(-, \mathcal{Y})$ to the short exact sequence $0 \rightarrow Y \rightarrow$ $Z \rightarrow X \rightarrow 0$, we obtain the exact sequence

$$
\operatorname{Hom}_{\mathcal{X}}(Y, \mathcal{Y}) \rightarrow \operatorname{Ext}_{\mathcal{X}}^{1}(X, \mathcal{Y}) \rightarrow \operatorname{Ext}_{\mathcal{X}}^{1}(Z, \mathcal{Y})=0 .
$$

Thus $\operatorname{Ext}_{\mathcal{X}}^{1}(X, \mathcal{Y})$ is a finitely generated $\mathcal{Y}^{\text {op }}$-module.

(ii) $\Rightarrow$ (i): There exists a projective cover $\varphi: \operatorname{Hom}_{\mathcal{X}}(Y, \mathcal{Y}) \rightarrow \operatorname{Ext}_{\mathcal{X}}^{1}(X, \mathcal{Y})$ since $\mathcal{Y}$ is Krull-Schmidt. Let

$$
0 \rightarrow Y \stackrel{f}{\longrightarrow} Z \stackrel{g}{\longrightarrow} X \rightarrow 0
$$

be a short exact sequence represented by $\varphi\left(\operatorname{id}_{Y}\right) \in \operatorname{Ext}_{\mathcal{X}}^{1}(X, Y)$. Since $\varphi$ is right minimal, $f$ belongs to $\operatorname{rad} \mathcal{X}$, and hence $g$ is right minimal. To prove $\operatorname{Ext}_{\mathcal{X}}^{1}(Z, \mathcal{Y})=0$, it suffices to show that any exact sequence

$$
0 \rightarrow Y^{\prime} \rightarrow W \stackrel{s}{\longrightarrow} Z \rightarrow 0
$$

with $Y^{\prime} \in \mathcal{Y}$ splits. We have the following commutative diagram of exact sequences:

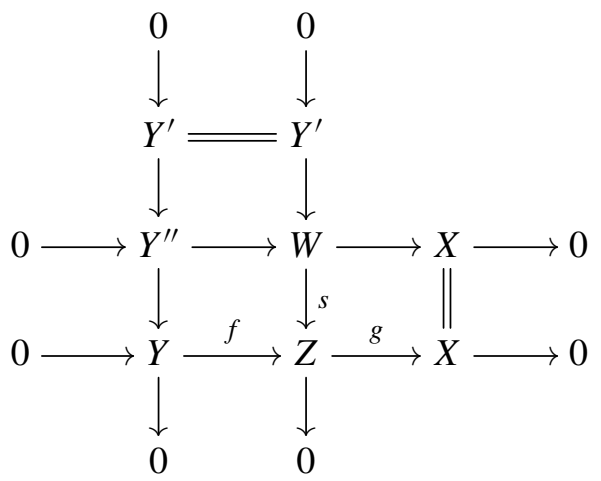

where $Y^{\prime \prime} \in \mathcal{Y}$ because $\mathcal{Y}$ is extension-closed. As $\varphi$ is an epimorphism, we have the following commutative diagram of exact sequences:

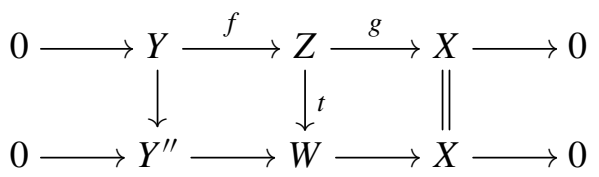

As $g$ is right minimal, $t s: Z \rightarrow Z$ is invertible. Therefore the sequence (4-1) splits.

Lemma 4.5. Suppose that $\operatorname{Ext}_{\mathcal{E}}^{1}(\mathcal{A}, \mathcal{B})=0$. Then the functor $F: \mathcal{E}_{2} \rightarrow \mathcal{B}$ is exact bijective. 
Proof. Let $X, Y \in \mathcal{E}_{2}$. By applying $\operatorname{Hom}_{\mathcal{E}}(X,-)$ to the short exact sequence $0 \rightarrow T Y \rightarrow Y \rightarrow F Y \rightarrow 0$, we have the isomorphism

$$
\operatorname{Ext}_{\mathcal{E}}^{1}(X, Y) \cong \operatorname{Ext}_{\mathcal{E}}^{1}(X, F Y),
$$

as $\operatorname{Ext}_{\mathcal{E}}^{i}(X, T Y)=0$ holds for $i=1,2$. Applying $\operatorname{Hom}_{\mathcal{E}}(-, F Y)$ to the short exact sequence $0 \rightarrow T X \rightarrow X \rightarrow F X \rightarrow 0$, we have an isomorphism

$$
\operatorname{Ext}_{\mathcal{E}}^{1}(F X, F Y) \cong \operatorname{Ext}_{\mathcal{E}}^{1}(X, F Y)
$$

as $\operatorname{Ext}_{\mathcal{E}}^{i}(T X, F Y)=0$ holds for $i=0,1$. Thus we have

$$
\operatorname{Ext}_{\mathcal{B}}^{1}(F X, F Y)=\operatorname{Ext}_{\mathcal{E}}^{1}(F X, F Y) \cong \operatorname{Ext}_{\mathcal{E}}^{1}(X, Y) .
$$

Lemma 4.6. Suppose that any object in $\mathcal{A}$ is projective in $\mathcal{E}$. Then $\mathcal{E}_{2} /[\mathcal{A}]$ inherits canonically the exact structure of $\mathcal{E}_{2}$, and the functor $F: \mathcal{E}_{2} /[\mathcal{A}] \rightarrow \mathcal{B}$ is exact bijective.

Proof. Any object $X \in \mathcal{E}$ has a right $\mathcal{A}$-approximation $T X \rightarrow X$ which is a categorical monomorphism, and we have

$$
\operatorname{Ext}_{\mathcal{E}}^{1}\left(\mathcal{A}, \mathcal{E}_{2}\right)=\operatorname{Ext}_{\mathcal{E}}^{1}\left(\mathcal{E}_{2}, \mathcal{A}\right)=0
$$

by our assumptions. Therefore Corollary 3.8(c) gives an exact structure on $\mathcal{E}_{2} /[\mathcal{A}]$. Applying Lemma 4.5, we have

$$
\operatorname{Ext}_{\mathcal{B}}^{1}(F X, F Y) \cong \operatorname{Ext}_{\mathcal{E}_{2}}^{1}(X, Y)=\operatorname{Ext}_{\mathcal{E}_{2} /[\mathcal{A}]}^{1}(X, Y),
$$

which shows the assertion.

4B. When there is a torsion pair $(\mathcal{B}, \mathcal{C})$. In this subsection, we further assume $(\mathcal{B}, \mathcal{C})$ is a torsion pair in $\mathcal{E}$ for

$$
\mathcal{C}:=\left\{X \in \mathcal{E} \mid \operatorname{Hom}_{\mathcal{E}}(\mathcal{B}, X)=0\right\} .
$$

The following result gives a description of the image of the functor $F: \mathcal{C} \rightarrow \mathcal{B}$.

Theorem 4.7. Assume that the following conditions are satisfied:

- $\mathcal{B}$ is an abelian category whose exact structure is compatible with that of $\mathcal{E}$.

- $\operatorname{Ext}_{\mathcal{E}}^{1}(\mathcal{B}, A)$ is a finitely generated $\mathcal{B}$-module for any $A \in \mathcal{A} \cap \mathcal{C}$.

Then we have the following assertions.

(a) For any $A \in \mathcal{A} \cap \mathcal{C}$, there exists a short exact sequence

$$
0 \rightarrow A \rightarrow C^{A} \rightarrow U^{A} \rightarrow 0
$$

with $U^{A} \in \mathcal{B}, C^{A} \in \mathcal{C}$ and $\operatorname{Ext}_{\mathcal{E}}^{1}\left(\mathcal{B}, C^{A}\right)=0$. Moreover, it is unique up to isomorphism. 
(b) Let $\mathcal{D}:=\operatorname{Sub}\left\{U^{A} \mid A \in \mathcal{A} \cap \mathcal{C}\right\}$. Then $F: \mathcal{E} \rightarrow \mathcal{B}$ induces a dense functor $F: \mathcal{C} \rightarrow \mathcal{D}$.

Assume $\operatorname{Ext}_{\mathcal{E}}^{2}(\mathcal{B}, \mathcal{A} \cap \mathcal{C})=0$.

(c) $U^{A}$ is an injective object in $\mathcal{B}$ for any $A \in \mathcal{A} \cap \mathcal{C}$.

(d) $\mathcal{D}$ is closed under taking extensions in $\mathcal{E}$, and therefore forms an exact category.

(e) Assume $\mathcal{C} \subset \mathcal{E}_{2}$ and that any object in $\mathcal{A}$ is projective in $\mathcal{E}$. Then $\mathcal{C} /[\mathcal{A}]$ inherits canonically the exact structure of $\mathcal{C}$ and $F: \mathcal{C} /[\mathcal{A}] \rightarrow \mathcal{D}$ is an equivalence of exact categories.

Proof. (a) By the dual of Lemma 4.4, we get a short exact sequence

$$
0 \rightarrow A \stackrel{f}{\longrightarrow} X \stackrel{g}{\longrightarrow} U^{A} \rightarrow 0
$$

for some $U^{A} \in \mathcal{B}$ such that $\operatorname{Ext}_{\mathcal{E}}^{1}(\mathcal{B}, X)=0$ and with $f$ left minimal. We only have to prove $X \in \mathcal{C}$. Since $(\mathcal{B}, \mathcal{C})$ is a torsion pair, there exists an exact sequence

$$
0 \rightarrow B \stackrel{i}{\rightarrow} X \rightarrow C \rightarrow 0
$$

with $B \in \mathcal{B}$ and $C \in \mathcal{C}$. Now we consider the following commutative diagram, where Ker $i g$ exists in $\mathcal{B}$ by our assumption:

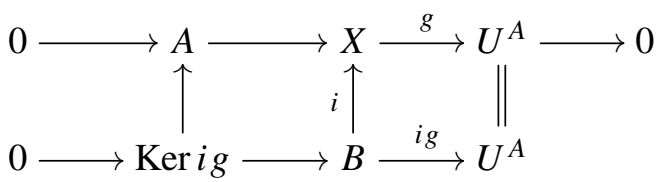

Since $A \in \mathcal{C}$, we have Kerig $=0$. Thus $i g$ is a monomorphism, and we can form the following commutative diagram with Coker $i g \in \mathcal{B}$ by our assumption:

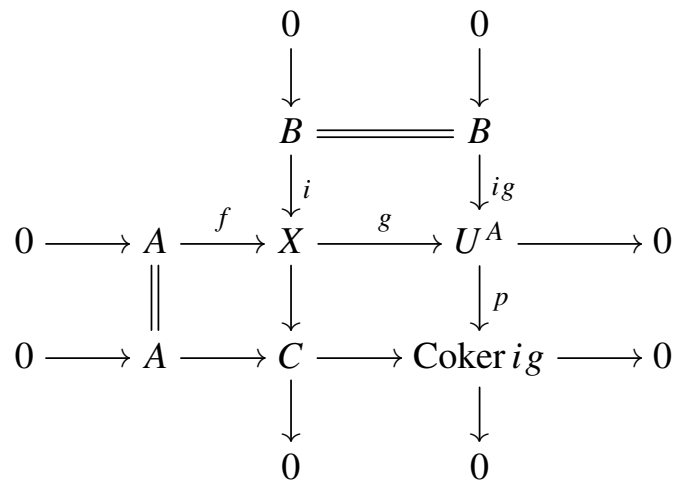

The upper horizontal sequence gives a projective cover $\varphi: \operatorname{Hom}_{\mathcal{E}}\left(\mathcal{B}, U^{A}\right) \rightarrow$ $\operatorname{Ext}_{\mathcal{E}}^{1}(\mathcal{B}, A)$ (see the proof of Lemma 4.4). The lower horizontal sequence gives a morphism $\psi: \operatorname{Hom}_{\mathcal{E}}(\mathcal{B}$, Coker $i g) \rightarrow \operatorname{Ext}_{\mathcal{E}}^{1}(\mathcal{B}, A)$, which is an epimorphism since 
$\varphi=\operatorname{Hom}_{\mathcal{E}}(\mathcal{B}, p) \psi$. Since Coker $i g \in \mathcal{B}$ and $\varphi$ is a projective cover, $p$ has to be an isomorphism. Thus we have $B=0$ and $X \cong C \in \mathcal{C}$.

As $\mathcal{B} \cap \mathcal{C}=0$, the morphism $A \rightarrow C^{A}$ is left minimal and it implies easily the uniqueness.

(b) First we prove $F(\mathcal{C}) \subset \mathcal{D}$. For any $C \in \mathcal{C}$, there exists an exact sequence $0 \rightarrow A \rightarrow C \rightarrow B \rightarrow 0$ with $B=F C \in \mathcal{B}$ and $A=T C \in \mathcal{A}$. Clearly we have $A \in \mathcal{A} \cap \mathcal{C}$. Let $0 \rightarrow A \rightarrow C^{A} \rightarrow U^{A} \rightarrow 0$ be the exact sequence in (a). Then we have a commutative diagram

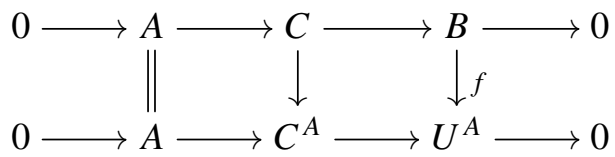

By our assumption, $f$ has a kernel $g: \operatorname{Ker} f \rightarrow B$ in $\mathcal{E}$ with $\operatorname{Ker} f \in \mathcal{B}$. Since the above diagram is pullback, $g$ factors through $C \in \mathcal{C}$. Thus $g=0$ holds, and hence $f$ is a monomorphism. Therefore $0 \rightarrow B \stackrel{f}{\longrightarrow} U^{A} \rightarrow$ Coker $f \rightarrow 0$ is a short exact sequence in $\mathcal{E}$ by our assumption, and $B \in \mathcal{D}$ holds.

Next we prove that the functor $F: \mathcal{B} \rightarrow \mathcal{D}$ is dense. For any $D \in \mathcal{D}$, there exist exact sequences

$$
0 \rightarrow D \rightarrow U^{A} \rightarrow X \rightarrow 0 \text { and } 0 \rightarrow A \rightarrow C^{A} \rightarrow U^{A} \rightarrow 0
$$

with $A \in \mathcal{A} \cap \mathcal{C}, U^{A} \in \mathcal{B}, C^{A} \in \mathcal{C}$ and $\operatorname{Ext}_{\mathcal{E}}^{1}\left(\mathcal{B}, C^{A}\right)=0$. Then we have a commutative diagram

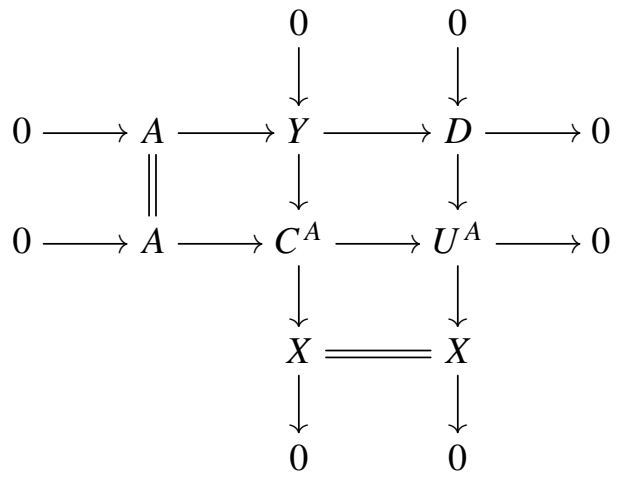

of exact sequences. Since $C^{A} \in \mathcal{C}$, we have $Y \in \mathcal{C}$ by the middle vertical sequence. Therefore $D=F Y$ belongs to $F(\mathcal{C})$.

(c) Applying $\operatorname{Hom}_{\mathcal{E}}(\mathcal{B},-)$ to the short exact sequence $0 \rightarrow A \rightarrow C^{A} \rightarrow U^{A} \rightarrow 0$, we have an exact sequence

$$
0=\operatorname{Ext}_{\mathcal{E}}^{1}\left(\mathcal{B}, C^{A}\right) \rightarrow \operatorname{Ext}_{\mathcal{E}}^{1}\left(\mathcal{B}, U^{A}\right) \rightarrow \operatorname{Ext}_{\mathcal{E}}^{2}(\mathcal{B}, A)=0 .
$$

Therefore $\operatorname{Ext}_{\mathcal{E}}^{1}\left(\mathcal{B}, U^{A}\right)=0$; that is, $U^{A}$ is injective in $\mathcal{B}$. 
(d) This is an immediate consequence of (c) and the horseshoe lemma.

(e) By Theorem 4.1(a) and (d), the functor $F: \mathcal{E}_{2} /[\mathcal{A}] \rightarrow \mathcal{B}$ is fully faithful and exact bijective. By $\mathcal{C} \subset \mathcal{E}_{2}$, using (b) and (d), we have an equivalence $F: \mathcal{C} /[\mathcal{A}] \rightarrow \mathcal{D}$ of exact categories.

4C. Frobenius properties. As in Section $4 \mathrm{~B}$, we suppose that $(\mathcal{B}, \mathcal{C})$ is a torsion pair. We define $\mathcal{U}:=\operatorname{add}\left\{U^{A} \mid A \in \mathcal{A} \cap \mathcal{C}\right\}$ and as in Theorem 4.7, $\mathcal{D}:=$ Sub $\mathcal{U}$. The following result gives a sufficient condition for the categories $\mathcal{C}$ and $\mathcal{D}$ to be Frobenius.

Theorem 4.8. Assume that the following conditions are satisfied:

- $\mathcal{B}$ is an abelian category whose exact structure is compatible with that of $\mathcal{E}$ and has enough projective objects and enough injective objects.

- $\mathcal{A} \subset \mathcal{C}$ holds, and any object in $\mathcal{A}$ is projective in $\mathcal{E}$ and injective in $\mathcal{C}$.

- $\operatorname{Ext}_{\mathcal{E}}^{1}(\mathcal{B}, A)$ is a finitely generated $\mathcal{B}$-module for any $A \in \mathcal{A}$.

- $\operatorname{Ext}_{\mathcal{E}}^{1}(P, \mathcal{A})$ is a finitely generated $\mathcal{A}^{\mathrm{op}}$-module for any projective object $P$ in $\mathcal{B}$.

Then we have the following assertions:

(a) $\mathcal{E}$ has enough projective objects and enough injective objects. Moreover, the following conditions are equivalent:

(i) Projective objects of $\mathcal{B}$ and $\mathcal{D}$ coincide.

(ii) Projective objects of $\mathcal{C}$ and $\mathcal{E}$ coincide.

Suppose that the equivalent conditions in (a) are satisfied. Then the following assertions hold:

(b) $\mathcal{C}$ and $\mathcal{D}$ have enough projective objects.

(c) Any object in $\mathcal{A}$ has injective dimension at most 1 in $\mathcal{E}$. Therefore all assertions in Theorem 4.7 hold.

(d) The following conditions are equivalent:

(i) $\mathcal{C}$ is a Frobenius category whose exact structure is compatible with that of $\mathcal{E}$.

(ii) $\mathcal{D}$ is a Frobenius category whose exact structure is compatible with that of $\mathcal{E}$.

(iii) Any object in $\mathcal{U}$ is projective-injective in $\mathcal{B}$. Moreover, each projective object of $\mathcal{B}$ has injective dimension at most 1 and each injective object of $\mathcal{B}$ has projective dimension at most 1 .

(e) If the conditions in (d) are satisfied, then the category of projective-injective objects in $\mathcal{B}$ is $\mathcal{U}$.

We start with preparing the following: 
Lemma 4.9. For any projective object $P$ in $\mathcal{B}$, there exists a projective object $X$ in $\mathcal{E}$ such that $P=F X$.

Proof. By Lemma 4.4, there exists a short exact sequence $0 \rightarrow A \rightarrow X \rightarrow P \rightarrow 0$ with $A \in \mathcal{A}$ and $\operatorname{Ext}_{\mathcal{E}}^{1}(X, \mathcal{A})=0$. Applying $\operatorname{Hom}_{\mathcal{E}}(-, \mathcal{B})$, we have an exact sequence

$$
0=\operatorname{Ext}_{\mathcal{E}}^{1}(P, \mathcal{B}) \rightarrow \operatorname{Ext}_{\mathcal{E}}^{1}(X, \mathcal{B}) \rightarrow \operatorname{Ext}_{\mathcal{E}}^{1}(A, \mathcal{B})=0 .
$$

Thus $\operatorname{Ext}_{\mathcal{E}}^{1}(X, \mathcal{B})=0$ holds. Since $\operatorname{Ext}_{\mathcal{E}}^{1}(X, \mathcal{A})=0$, we have $\operatorname{Ext}_{\mathcal{E}}^{1}(X, \mathcal{E})=0$. Thus $X$ is a projective object in $\mathcal{E}$ satisfying $P=F X$.

Now we are ready to prove Theorem 4.8 .

Proof of Theorem 4.8. (a) For any $X \in \mathcal{E}$, there exists a short exact sequence $0 \rightarrow A \rightarrow X \rightarrow B \rightarrow 0$ with $A=T X \in \mathcal{A}$ and $B=F X \in \mathcal{B}$. Then $A$ is projective in $\mathcal{E}$ by our assumption. Thanks to the horseshoe lemma, to show that $X$ has a projective cover in $\mathcal{E}$, it suffices to show that any $B \in \mathcal{B}$ has a projective cover in $\mathcal{E}$.

By our assumption, there exists a projective cover $f: P \rightarrow B$ in $\mathcal{B}$. By Lemma 4.9, there exists a projective cover $g: P^{\prime} \rightarrow P$ in $\mathcal{E}$. Then the composition $g f: P^{\prime} \rightarrow B$ gives a projective cover of $B$ in $\mathcal{E}$.

In the same way, to prove that $\mathcal{E}$ has enough injective objects, it is enough to prove that any $A \in \mathcal{A}$ and any $B \in \mathcal{B}$ admits an injective hull in $\mathcal{E}$. For $B \in \mathcal{B}$, it admits an injective hull $I$ in $\mathcal{B}$. As $(\mathcal{A}, \mathcal{B})$ is a torsion pair in $\mathcal{E}$ and $\operatorname{Ext}_{\mathcal{E}}^{1}(\mathcal{A}, \mathcal{E})=0$, we know $I$ is injective in $\mathcal{E}$. For $A \in \mathcal{A}$, the object $C^{A}$ defined in Theorem 4.7(a) is an injective hull of $A$ by the same argument. So we proved that $\mathcal{E}$ has enough injective objects.

(ii) $\Rightarrow$ (i): Suppose that projective objects of $\mathcal{C}$ and $\mathcal{E}$ coincide.

Let $P$ be a projective object in $\mathcal{B}$. By Lemma 4.9 , there exists a projective object $X$ in $\mathcal{E}$ such that $P=F X$. Since $X$ belongs to $\mathcal{C}$ by our assumption, we have $P \in F(\mathcal{C}) \subset \mathcal{D}$. Thus $P$ is a projective object in $\mathcal{D}$.

Let $P$ be a projective object in $\mathcal{D}$. Since $\mathcal{B}$ has enough projective objects by our assumption, there exists a projective cover $f: X \rightarrow P$ in $\mathcal{B}$. Since $X$ belongs to $\mathcal{D}$ by the above argument, $f$ splits. Thus $P$ is projective in $\mathcal{B}$.

(i) $\Rightarrow$ (ii): Suppose that projective objects of $\mathcal{B}$ and $\mathcal{D}$ coincide.

Let $P$ be a projective object in $\mathcal{E}$. Let $0 \rightarrow X \rightarrow P^{\prime} \stackrel{f}{\longrightarrow} F P \rightarrow 0$ be an exact sequence with a projective object $P^{\prime}$ in $\mathcal{B}$. Then $P^{\prime} \in \mathcal{D}$ by our assumption. By Theorem 4.7(b), there exists an exact sequence $0 \rightarrow A \stackrel{i}{\longrightarrow} C \stackrel{p}{\longrightarrow} P^{\prime} \rightarrow 0$ with $A \in \mathcal{A}$ and $C \in \mathcal{C}$. Since $\operatorname{Ext}_{\mathcal{E}}^{1}(C, \mathcal{A})=0$ holds by our assumption, we have a commutative diagram:

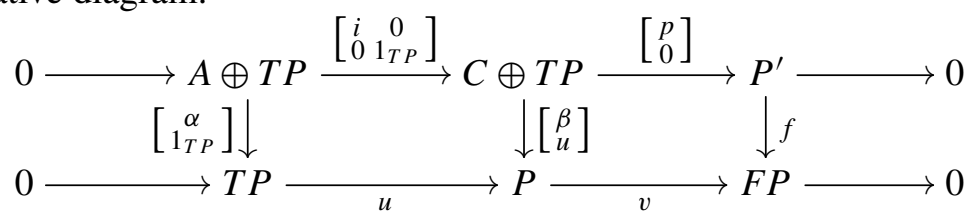


As $\left[\begin{array}{c}\alpha \\ 1_{T P}\end{array}\right]$ and $f$ are (admissible) epimorphisms, $\left[\begin{array}{l}\beta \\ u\end{array}\right]$ is also one. Since $P$ is projective in $\mathcal{E}$, we know $\left[\begin{array}{l}\beta \\ u\end{array}\right]$ splits. Thus $P$ is a direct summand of $C \oplus T P$, which belongs to $\mathcal{C}$ by our assumption $T P \in \mathcal{A} \subset \mathcal{C}$.

Conversely, let $Q$ be a projective object in $\mathcal{C}$. Let us consider its projective cover $P$ in $\mathcal{E}$. We get the short exact sequence

$$
0 \rightarrow \Omega_{\mathcal{E}} Q \rightarrow P \rightarrow Q \rightarrow 0 .
$$

According to the previous discussion, $P \in \mathcal{C}$. Thus, we get that $\Omega_{\mathcal{E}} Q \in \mathcal{C}$. Hence, as $Q$ is projective in $\mathcal{C}$, the short exact sequence splits and $Q$ is projective in $\mathcal{E}$.

(b) We now suppose that the conditions in (a) are satisfied. Since $\mathcal{E}$ has enough projective objects which belong to $\mathcal{C}$, we get that $\mathcal{C}$ has enough projective objects. By a similar argument, $\mathcal{D}$ has enough projective objects.

(c) All projective objects of $\mathcal{E}$ belong to $\mathcal{C}$ by our assumption. Therefore $\Omega_{\mathcal{E}}(\mathcal{E}) \subset \mathcal{C}$ holds. Since any object in $\mathcal{A}$ is injective in $\mathcal{C}$ by our assumption, we have

$$
\operatorname{Ext}_{\mathcal{E}}^{2}(\mathcal{E}, \mathcal{A})=\operatorname{Ext}_{\mathcal{E}}^{1}\left(\Omega_{\mathcal{E}}(\mathcal{E}), \mathcal{A}\right)=0 .
$$

Thus the first assertion follows. In particular we have $\operatorname{Ext}_{\mathcal{E}}^{2}(\mathcal{B}, \mathcal{A} \cap \mathcal{C})=0$, and the second assertion follows.

(d)-(e) Thanks to Theorems 3.6 and 4.7(e), $F: \mathcal{C} \rightarrow \mathcal{C} /[\mathcal{A}] \rightarrow \mathcal{D}$ is exact bijective. So $\mathcal{C}$ is Frobenius if and only if $\mathcal{D}$ is Frobenius by Remark 3.3. Hence (i) $\Leftrightarrow$ (ii) in (d) is proven. The remaining assertions follow by applying Proposition 3.9 to $\mathcal{B}$.

\section{Equivalences arising from orders and their idempotents}

As in Section 2A, let $R$ be a complete discrete valuation ring and $K$ be its field of fractions. Fix an $R$-order $A$. Consider functors

$$
D_{i}:=\operatorname{Ext}_{R}^{1-i}(-, R): \bmod A \leftrightarrow \bmod A^{\text {op }}
$$

for $i=0,1$. They restrict to dualities

$D_{1}=\operatorname{Hom}_{R}(-, R): \mathrm{CM} A \stackrel{\sim}{\longleftrightarrow} \mathrm{CM} A^{\mathrm{op}} \quad$ and $\quad D_{0}=\operatorname{Ext}_{R}^{1}(-, R):$ f.I. $A \stackrel{\sim}{\longleftrightarrow}$ f.I. $A^{\text {op }}$

and satisfy $D_{0}(\mathrm{CM} A)=D_{1}(\mathrm{f} . \mathrm{I} . A)=0$. In view of the characterizations of CM $A$ given at the beginning of Section 2, it is immediate that $\mathrm{CM} A$ admits the projective generator $A$ and the injective cogenerator $D_{1} A$. Since the injective resolution of the $R$-module $R$ is given by $0 \rightarrow R \rightarrow K \rightarrow K / R \rightarrow 0$, we get an isomorphism $D_{0} \cong \operatorname{Hom}_{R}(-, K / R)$ on f.I. $A$. Recall the following useful lemma:

Lemma 5.1. If $X \in \mathrm{CM} A$, then we have a monomorphism $X \hookrightarrow X \otimes_{R} K$ and $\operatorname{Ext}_{A}^{1}\left(\right.$ f.I. $\left.A, X \otimes_{R} K\right)=0$. 
Proof. For $Y \in$ f.I. $A$, let $E:=\operatorname{Ext}_{A}^{1}\left(Y, X \otimes_{R} K\right)$. Since $X \otimes_{R} K$ is a $K$-vector space, so is $E$. Since $Y$ is annihilated by some nonzero element in $R$, so is $E$. These imply $E=0$.

For an object $X \in \mathrm{CM} A$, let corad $X \in \mathrm{CM} A$ be maximal among $A$-submodules $Y$ of $X \otimes_{R} K$ such that $X \subset Y$ and $Y / X$ is semisimple. We define cotop $X:=$ $(\operatorname{corad} X) / X$. Notice that $X \otimes_{R} K$ is not finitely generated as an $A$-module (so $\left.X \otimes_{R} K \notin \mathrm{CM} A\right)$ if $X \in \mathrm{CM} A$ is nonzero. Notice also that $D_{1}\left(X \otimes_{R} K\right)=0$.

We often use the following lemma:

Lemma 5.2. Let $X \in \mathrm{CM} A$. The following hold:

(a) We have cotop $X=\operatorname{soc}\left(X \otimes_{R}(K / R)\right)$.

(b) The functor $D_{1}$ induces an order-reversing bijection

$$
\left\{X \subset Y \subset X \otimes_{R} K \mid Y / X \in \text { f.I. } A\right\} \stackrel{1-1}{\longleftrightarrow}\left\{Y^{\prime} \subset D_{1} X \mid\left(D_{1} X\right) / Y^{\prime} \in \text { f.l. } A^{\text {op }}\right\} .
$$

(c) There are isomorphisms corad $X \cong D_{1} \operatorname{rad} D_{1} X$ and cotop $X \cong D_{0}$ top $D_{1} X$ of A-modules.

(d) If $0 \rightarrow X \rightarrow Y \rightarrow S \rightarrow 0$ is a short exact sequence with $Y \in \mathrm{CM} A$ and a semisimple A-module $S$, then there is a unique canonical commutative diagram

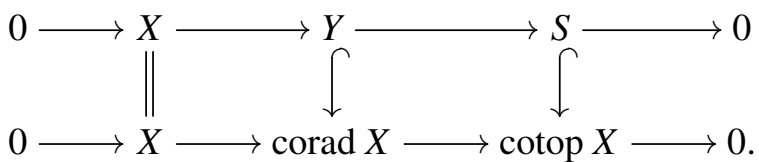

(e) For a simple A-module $S$, we have $\operatorname{Ext}_{A}^{1}(S, X) \neq 0$ if and only if $S$ is a direct summand of $\operatorname{cotop} X$.

Proof. Parts (a) and (b) are immediate and the first isomorphism of (c) is a consequence of (b). The second isomorphism of (c) is obtained by applying $\operatorname{Hom}_{R}(-, R)$ to the short exact sequence $0 \rightarrow \operatorname{rad} D_{1} X \rightarrow D_{1} X \rightarrow$ top $D_{1} X \rightarrow 0$. For (d), applying the functor $-\otimes_{R} K$ to the short exact sequence, we get $X \otimes_{R} K \cong Y \otimes_{R} K$. Therefore $X \subset Y \subset X \otimes_{R} K$. By the maximality of corad $X$, we have $Y \subset \operatorname{corad} X$ and the result follows.

(e) The implication " $\Leftarrow$ " is immediate. Let us show " $\Rightarrow$ ". Consider a nonsplit exact sequence $0 \rightarrow X \rightarrow Y \rightarrow S \rightarrow 0$. For any simple module $S^{\prime}$, applying $\operatorname{Hom}_{A}\left(S^{\prime},-\right)$, we get an exact sequence $0 \rightarrow \operatorname{Hom}_{A}\left(S^{\prime}, Y\right) \rightarrow \operatorname{Hom}_{A}\left(S^{\prime}, S\right) \rightarrow \operatorname{Ext}_{A}^{1}\left(S^{\prime}, X\right)$. It is easy to conclude in any case that $\operatorname{Hom}_{A}\left(S^{\prime}, Y\right)=0$, so $Y \in \mathrm{CM} A$. Therefore, we can apply (d) so $S$ is a summand of $\operatorname{cotop} X$.

For logical reasons, we give the proof of Theorem 2.1 after that of Theorem 2.2. 
5A. Proof of Theorem 2.2. As in Theorem 2.2, we consider an idempotent $e$ of an $R$-order $A$ such that $B:=A /(e)$ has finite length over $R$. As $\bmod B \subset$ f.I. $A$, $D_{0}$ restricts to a duality $\bmod B \stackrel{\sim}{\longleftrightarrow} \bmod B^{\text {op }}$. We will separate the proof in five statements.

Proposition 5.3. We have a torsion pair $(\operatorname{add} A e, \bmod B)$ in $\bmod _{e} A$.

Proof. Since $B=A /(e)$, we have $\operatorname{Hom}_{A}(\operatorname{add} A e, \bmod B)=0$. For any $X \in \bmod _{e} A$, we have an exact sequence

$$
A e \otimes_{e A e} e X \stackrel{f}{\longrightarrow} X \rightarrow B \otimes_{A} X \rightarrow 0
$$

in $\bmod _{e} A$. Since $e X \in \operatorname{proj}(e A e)$, we have $A e \otimes_{e A e} e X \in \operatorname{add} A e$. Multiplying the sequence (5-1) by $e$ on the left, we see that $e \operatorname{Ker} f=0$ so $\operatorname{Ker} f$ is $\operatorname{in} \bmod B$. On the other hand, Ker $f$ is a submodule of $A e \otimes_{e A e} e X \in \operatorname{add} A e$, so Ker $f \in \operatorname{CM} A$. Consequently we have $\operatorname{Ker} f=0$. Now the sequence (5-1) shows the desired assertion.

Thanks to Proposition 5.3, we have two functors $T: \bmod _{e} A \rightarrow$ add $A e$ and $F: \bmod _{e} A \rightarrow \bmod B$ and a functorial exact sequence $0 \rightarrow T X \rightarrow X \rightarrow F X \rightarrow 0$ for $X \in \bmod _{e} A$. We prove the following easy statement:

Lemma 5.4. If $X \in \mathrm{CM}_{e} A$, then $F X \subset \operatorname{Hom}_{A}\left(B, T X \otimes_{R}(K / R)\right) \subset T X \otimes_{R}(K / R)$ and $\operatorname{soc} F X \subset \operatorname{cotop} T X$.

Proof. The inclusion $\operatorname{Hom}_{A}\left(B, T X \otimes_{R}(K / R)\right) \subset T X \otimes_{R}(K / R)$ is obvious. Applying $-\otimes_{R} K$ on the short exact sequence $0 \rightarrow T X \rightarrow X \rightarrow F X \rightarrow 0$, we get that $T X \otimes_{R} K \cong X \otimes_{R} K$ so $X \subset T X \otimes_{R} K$ canonically. Thus we get a commutative diagram of short exact sequences

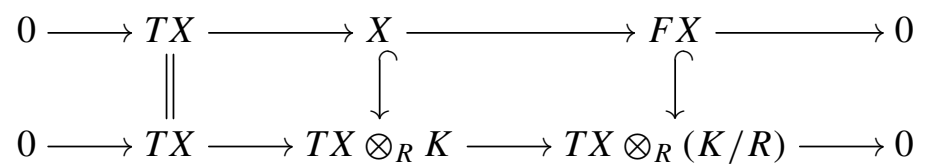

where the second line is obtained by applying $T X \otimes_{R}-$ to $0 \rightarrow R \rightarrow K \rightarrow$ $K / R \rightarrow 0$. Thus $F X \subset T X \otimes_{R}(K / R)$. As $F X \in \bmod B$, we deduce that $F X \subset$ $\operatorname{Hom}_{A}\left(B, T X \otimes_{R}(K / R)\right)$. The latter assertion follows from Lemma 5.2(a).

Proposition 5.5. We have a torsion pair $\left(\bmod B, \mathrm{CM}_{e} A\right)$ in $\bmod _{e} A$.

Proof. Since any $X \in \bmod B$ has finite length, we have $\operatorname{Hom}_{A}\left(\bmod B, \mathrm{CM}_{e} A\right)=0$. For any $X \in \bmod _{e} A$, there exists an exact sequence

$$
0 \rightarrow T \rightarrow X \rightarrow F \rightarrow 0
$$

in $\bmod A$ such that length ${ }_{R} T<\infty$ and $F \in \mathrm{CM} A$. Multiplying $e$ from the left, we have an exact sequence

$$
0 \rightarrow e T \rightarrow e X \rightarrow e F \rightarrow 0
$$


with length ${ }_{R}(e T)<\infty$ and $e X \in \operatorname{proj}(e A e)$. Thus $e T=0$ holds, and we have $T \in \bmod B$. On the other hand, $e F=e X \in \operatorname{proj}(e A e)$ shows $F \in \mathrm{CM}_{e} A$. Thus the assertion follows.

Now we can apply Theorems 4.1, 4.7 and 4.8 to

$$
\mathcal{E}:=\bmod _{e} A, \quad \mathcal{A}:=\operatorname{add} A e, \quad \mathcal{B}:=\bmod B \quad \text { and } \quad \mathcal{C}:=\mathrm{CM}_{e} A .
$$

In this context, it is possible to compute explicitly the short exact sequence given in Theorem 4.7(a). For $P \in$ add $A e$, let

$$
U^{P}:=\operatorname{Hom}_{A}\left(B, P \otimes_{R}(K / R)\right) \in \bmod B
$$

and define $U:=U^{A e}$. For any $X \in \mathrm{CM} A$, we define

$$
B \text {-cotop } X:=\operatorname{Hom}_{A}(B, \operatorname{cotop} X) \text {. }
$$

In other terms, $B$-cotop $X$ is the biggest $B$-module included in cotop $X$. We also define $B$-corad $X$ as the $A$-module satisfying

$X \subset B-\operatorname{corad} X \subset \operatorname{corad} X$ and $B$-cotop $X \cong(B-\operatorname{corad} X) / X$.

Lemma 5.6. Let $P \in$ add Ae. The following hold:

(a) There is a short exact sequence $0 \rightarrow P \rightarrow C^{P} \rightarrow U^{P} \rightarrow 0$ in $\bmod A$ with $C^{P} \in \mathrm{CM}_{e} A$ and $\operatorname{Ext}_{A}^{1}\left(\bmod B, C^{P}\right)=0$.

Conversely, if $0 \rightarrow P \rightarrow C^{\prime} \rightarrow U^{\prime} \rightarrow 0$ is a short exact sequence with $C^{\prime} \in \mathrm{CM}_{e} A, U^{\prime} \in \bmod B$ and $\operatorname{Ext}_{A}^{1}\left(\bmod B, C^{\prime}\right)=0$, then it is isomorphic to the above short exact sequence.

(b) We have an isomorphism $\operatorname{soc} U^{P} \cong B$-cotop $P$ of $B$-modules.

Proof. (a) Applying $P \otimes_{R}-$ to the short exact sequence $0 \rightarrow R \rightarrow K \rightarrow K / R \rightarrow 0$, we obtain the short exact sequence $0 \rightarrow P \rightarrow P \otimes_{R} K \rightarrow P \otimes_{R}(K / R) \rightarrow 0$ with $\operatorname{Ext}_{A}^{1}$ (f.I. $\left.A, P \otimes_{R} K\right)=0$ thanks to Lemma 5.1. Taking the pullback by the natural inclusion $U^{P} \subset P \otimes_{R}(K / R)$, we get the following commutative diagram of short exact sequences:

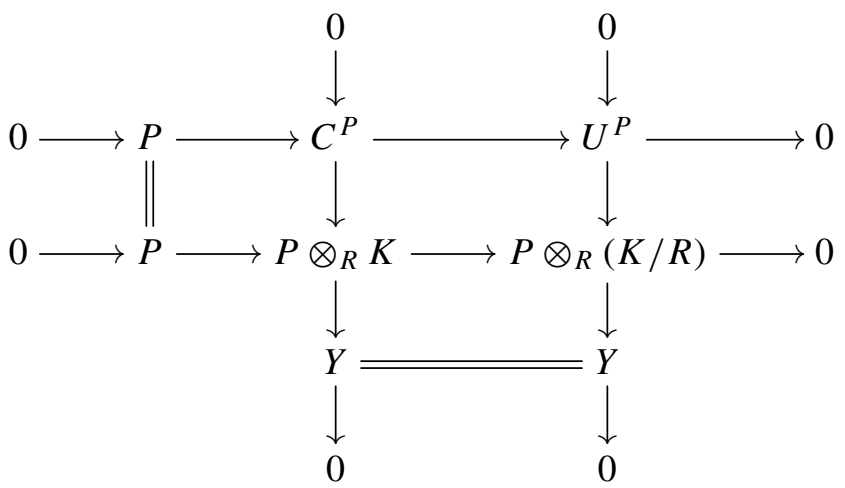


Since $U^{P}$ is the maximal $B$-module included in $P \otimes_{R}(K / R)$, and $\bmod B$ is closed under extensions in $\bmod A$, we get $\operatorname{Hom}_{A}(\bmod B, Y)=0$. Then applying $\operatorname{Hom}_{A}(\bmod B,-)$ to the second column, we find the exact sequence

$$
0=\operatorname{Hom}_{A}(\bmod B, Y) \rightarrow \operatorname{Ext}_{A}^{1}\left(\bmod B, C^{P}\right) \rightarrow \operatorname{Ext}_{A}^{1}\left(\bmod B, P \otimes_{R} K\right)=0 .
$$

Thus $\operatorname{Ext}_{A}^{1}\left(\bmod B, C^{P}\right)=0$ holds.

Now we prove the converse part. Applying $\operatorname{Hom}_{A}\left(U^{\prime},-\right)$ to the former sequence, we get a surjection $\operatorname{Hom}_{A}\left(U^{\prime}, U^{P}\right) \rightarrow \operatorname{Ext}_{A}^{1}\left(U^{\prime}, P\right)$ so there is a commutative diagram:

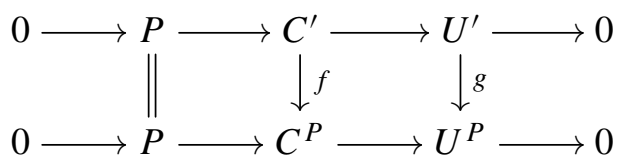

In the same way, there are $f^{\prime}: C^{P} \rightarrow C^{\prime}$ and $g^{\prime}: U^{P} \rightarrow U^{\prime}$ making a commutative diagram in the converse direction. Then $f f^{\prime}-\operatorname{id}_{C^{\prime}}$ factors through $U^{\prime}$, hence $f f^{\prime}=\operatorname{id}_{C^{\prime}}$. Similarly, $f^{\prime} f=\operatorname{id}_{C^{P}}$. Hence, $f$ and $g$ are isomorphisms.

(b) By Lemma 5.2, $\operatorname{cotop} P=\operatorname{soc}\left(P \otimes_{R}(K / R)\right)$. Applying $\operatorname{Hom}_{A}(B,-)$ to both sides, we obtain $\operatorname{Hom}_{A}(B, \operatorname{cotop} P)=\operatorname{Hom}_{A}\left(B, \operatorname{soc}\left(P \otimes_{R}(K / R)\right)\right)=\operatorname{soc} U^{P}$.

We are ready to prove Theorem 2.2.

Proof of Theorem 2.2. (a) This follows from Propositions 5.3 and 5.5.

(b) This follows from Theorem 4.1(a) and (b) as $\operatorname{Ext}_{A}^{1}(Y, A e)$ is a finitely generated right $(e A e)$-module for any $Y \in \bmod B$.

(c) Our assumption (E1) implies $\mathrm{CM}_{e} A \subset \mathcal{E}_{1}$. Thus the functor $F:\left(\mathrm{CM}_{e} A\right) /[A e] \rightarrow$ $\bmod B$ is fully faithful by (a). It gives an equivalence $F:\left(\mathrm{CM}_{e} A\right) /[A e] \rightarrow \operatorname{Sub} U$ by Theorem 4.7(b) and Lemma 5.6.

(d) This follows from (E2) and Theorem 4.7(c).

(e) Thanks to (E2), $\mathcal{E}_{1}=\mathcal{E}_{2}$ so, using Theorem 4.1(d), (2-1) and (2-2) are equivalences of exact categories.

(f) It is classical that Sub $U$ has enough projective objects and enough injective objects (see [Demonet and Iyama $\geq 2016$ ] for a detailed argument). Using (e) and Remark 3.3(b), it immediately implies that $\mathrm{CM}_{e} A$ has enough projective objects and enough injective objects. In the same way, as $\bmod B$ has enough injective objects and enough projective objects, $\mathcal{E}_{1}$ has the same property. Then $\bmod _{e} A$ has enough projective objects and enough injective objects by Theorem 4.8(a).

(g) For any $X \in \bmod _{e} A$, as $\left(\bmod B, C_{e} A\right)$ is a torsion pair, there is a short exact sequence

$$
0 \rightarrow Z \rightarrow X \rightarrow Y \rightarrow 0,
$$


where $Z \in \bmod B$ and $Y \in \mathrm{CM}_{e} A$. Applying $\operatorname{Hom}_{A}(-, A e)$ to this sequence, we find the exact sequence

$$
0=\operatorname{Ext}_{A}^{1}(Y, A e) \rightarrow \operatorname{Ext}_{A}^{1}(X, A e) \rightarrow \operatorname{Ext}_{A}^{1}(Z, A e) \rightarrow \operatorname{Ext}_{A}^{2}(Y, A e)=0 .
$$

So $X \in \mathcal{E}_{1}$ if and only if $\operatorname{Ext}_{A}^{1}(Z, A e)=0$. There is a short exact sequence

$$
0 \rightarrow \operatorname{soc} Z \rightarrow Z \rightarrow Z / \operatorname{soc} Z \rightarrow 0,
$$

and applying $\operatorname{Hom}_{A}(-, A e)$ to it, we find the exact sequence

$$
0 \rightarrow \operatorname{Ext}_{A}^{1}(Z / \operatorname{soc} Z, A e) \rightarrow \operatorname{Ext}_{A}^{1}(Z, A e) \rightarrow \operatorname{Ext}_{A}^{1}(\operatorname{soc} Z, A e) \rightarrow 0,
$$

so $\operatorname{Ext}_{A}^{1}(Z, A e)=0$ if and only if $\operatorname{Ext}_{A}^{1}(Z / \operatorname{soc} Z, A e)=\operatorname{Ext}_{A}^{1}(\operatorname{soc} Z, A e)=0$. By Lemma 5.2(e), for a simple $B$-module $S$, we have $\operatorname{Ext}_{A}^{1}(S, A e)=0$ if and only if $S$ is not a direct summand of $B$-cotop $A e$ if and only if $S \notin$ Sub $U$ if and only if $\operatorname{Hom}_{A}(P, S)=0$, where $P$ is the projective cover of $\operatorname{soc} U$ in $\bmod B$. As $Z$ is of finite length over $R$, an easy induction gives that $\operatorname{Ext}_{A}^{1}(Z, A e)=0$ if and only if $\operatorname{Hom}_{A}(P, Z)=0$ if and only if $\operatorname{Hom}_{A}(P, X)=0$.

In the following lemma, we give sufficient conditions for (E1) and (E2):

Lemma 5.7. (a) We have the implication $(E 2)^{+} \Rightarrow(E 2)$.

(b) If $A e=\operatorname{Hom}_{R}(g A, R)$ for some idempotent $g \in A$, then $(E 1)$ and $(E 2)^{+}$are satisfied.

(c) If (E1) is satisfied and $A \in \mathrm{CM}_{e} A$, then $(E 2)^{+}$is satisfied.

Proof. (a) This directly follows from Proposition 3.4.

(b) In this case, $\operatorname{Ext}_{A}^{1}(\mathrm{CM} A, A e)=0$, so (E1) is clearly satisfied. If $X \in \bmod A$, it is immediate that its syzygy $\Omega X$ is in $\mathrm{CM}_{A}$ so $\operatorname{Ext}_{A}^{2}(X, A e)=\operatorname{Ext}_{A}^{1}(\Omega X, A e)=0$. Therefore, (E2) ${ }^{+}$holds.

(c) For $X \in \bmod _{e} A$, consider the projective cover $0 \rightarrow \Omega X \rightarrow P \rightarrow X \rightarrow 0$. As $e X \in \operatorname{proj}(e A e)$, the short exact sequence $0 \rightarrow e \Omega X \rightarrow e P \rightarrow e X \rightarrow 0$ splits. Moreover, as $A \in \mathrm{CM}_{e} A$, we have $e P \in \operatorname{proj}(e A e)$ so $\Omega X \in \mathrm{CM}_{e} A$. So, by (E1), $\operatorname{Ext}_{A}^{2}(X, A e)=\operatorname{Ext}_{A}^{1}(\Omega X, A e)=0$ and $(\mathrm{E} 2)^{+}$holds.

We complete this subsection by giving basic relations between indecomposable injective objects of $\mathrm{CM}_{e} A$ and their $B$-cotops. Let

$$
\mathcal{O}:=\{P \in \text { ind } A e \mid B \text {-cotop } P \neq 0\} .
$$

Notice that part (a) of Lemma 5.8 is a generalization of a well-known property of cotops in $\mathrm{CM} A$.

Lemma 5.8. Let $I \in \mathrm{CM}_{e}$ A satisfying $\operatorname{Ext}_{A}^{1}\left(\mathrm{CM}_{e} A, I\right)=0$. Then the following hold:

(a) If I is indecomposable, then B-cotop I is either 0 or simple. 
(b) $B$-cotop $I=0$ if and only if $\operatorname{Ext}_{A}^{1}(\bmod B, I)=0$.

(c) For any short exact sequence $0 \rightarrow I \stackrel{i}{\rightarrow} X \stackrel{p}{\rightarrow} Y \rightarrow 0$, where $i$ is a radical map, $X \in \mathrm{CM}_{e} A$ and $Y \in \bmod _{e} A$, the map $i$ factors as $I \subset B$-corad $I \hookrightarrow X$ and $\operatorname{soc} Y \cong B$-cotop $I$.

(d) If (E1) is satisfied, there are commuting bijections

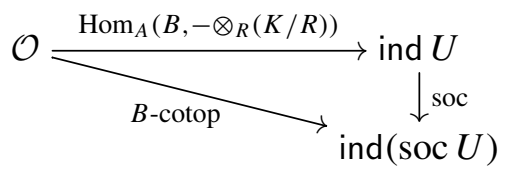

Proof. (a) Thanks to Lemma 5.2(c), $\operatorname{cotop} I \cong D_{0}$ top $D_{1} I$, so we only have to show that the $A^{\text {op }}$-module $\operatorname{Hom}_{A^{\text {op }}}\left(B\right.$, top $\left.D_{1} I\right)$ is 0 or simple. Suppose that $\operatorname{Hom}_{A^{\text {op }}}\left(B\right.$, top $\left.D_{1} I\right)$ is not 0 or simple. We have two distinct maximal submodules $X_{1}, X_{2} \subset D_{1} I$ such that $S_{1}:=\left(D_{1} I\right) / X_{1}$ and $S_{2}:=\left(D_{1} I\right) / X_{2}$ are simple $B^{\text {op }}$-modules. By applying $\operatorname{Hom}_{R}(-, R)$ on the short exact sequence $0 \rightarrow X_{1} \rightarrow D_{1} I \rightarrow S_{1} \rightarrow 0$, we get the short exact sequence

$$
0 \rightarrow I \stackrel{\iota_{1}}{\longrightarrow} D_{1} X_{1} \rightarrow D_{0} S_{1} \rightarrow 0,
$$

and therefore $e \iota_{1}: e I \rightarrow e\left(D_{1} X_{1}\right)$ is an isomorphism and $D_{1} X_{1} \in \mathrm{CM}_{e} A$. In the same way, $e \iota_{2}: e I \rightarrow e\left(D_{1} X_{2}\right)$ is an isomorphism and $D_{1} X_{2} \in \mathrm{CM}_{e} A$. We also get a nonsplit short exact sequence $0 \rightarrow Y \rightarrow X_{1} \oplus X_{2} \rightarrow D_{1} I \rightarrow 0$. Applying $D_{1}$ to it, we get a short exact sequence $0 \rightarrow I \rightarrow D_{1}\left(X_{1} \oplus X_{2}\right) \rightarrow D_{1} Y \rightarrow 0$. Multiplying by $e$, we get the short exact sequence

$$
0 \rightarrow e I \stackrel{\left[e \iota_{1} e \iota_{2}\right]}{\longrightarrow} e\left(D_{1} X_{1}\right) \oplus e\left(D_{1} X_{1}\right) \rightarrow e\left(D_{1} Y\right) \rightarrow 0,
$$

which splits as $e \iota_{1}$ and $e \iota_{2}$ are isomorphisms. Thus $0 \rightarrow I \rightarrow D_{1}\left(X_{1} \oplus X_{2}\right) \rightarrow$ $D_{1} Y \rightarrow 0$ is a nonsplit short exact sequence in $\mathrm{CM}_{e} A$. It is a contradiction as $\operatorname{Ext}_{A}^{1}\left(\mathrm{CM}_{e} A, I\right)=0$.

(b) Thanks to Lemma 5.2(e), a simple $B$-module $S$ is a direct summand of $B$-cotop $I$ if and only if $\operatorname{Ext}_{A}^{1}(S, I) \neq 0$. Thus $B$-cotop $I=0$ if and only if $\operatorname{Ext}_{A}^{1}(S, I)=0$ for any simple $B$-module $S$ if and only if $\operatorname{Ext}_{A}^{1}(\bmod B, I)=0$.

(c) Thanks to Proposition 5.3, soc $Y \in \bmod B$. Consider the sequence $0 \rightarrow I \rightarrow$ $p^{-1}(\operatorname{soc} Y) \rightarrow \operatorname{soc} Y \rightarrow 0$. Thanks to Lemma 5.2(d), we have soc $Y \hookrightarrow B$-cotop $I$.

We will prove, for each direct summand $I^{\prime}$ of $I$, that $B-\operatorname{corad} I^{\prime}\left(\subset X \otimes_{R} K\right)$ is included in $X$. Consider the short exact sequence $0 \rightarrow I^{\prime} \rightarrow X \rightarrow Y^{\prime} \rightarrow 0$ induced by the inclusion $I^{\prime} \subset I$. As $i$ is radical, this short exact sequence does not split and we get $Y^{\prime} \notin \mathrm{CM}_{e} A$ and $\operatorname{soc} Y^{\prime} \neq 0$. Pulling back $0 \rightarrow I^{\prime} \rightarrow X \rightarrow Y^{\prime} \rightarrow 0$ along $\operatorname{soc} Y^{\prime} \subset Y^{\prime}$, we get a short exact sequence $0 \rightarrow I^{\prime} \rightarrow X^{\prime} \rightarrow \operatorname{soc} Y^{\prime} \rightarrow 0$ with $X^{\prime} \subset X$ so $X^{\prime} \in \mathrm{CM}_{e} A$. Using (a) and Lemma 5.2(d), we obtain soc $Y^{\prime} \cong B$-cotop $I^{\prime}$ and 
therefore $X^{\prime}=B$-corad $I^{\prime} \subset X$. Finally $B$-corad $I \subset X$ and therefore $B$-cotop $I \hookrightarrow Y$. As $B$-cotop $I$ is semisimple, $B$-cotop $I \hookrightarrow \operatorname{soc} Y$. So $B$-cotop $I \cong \operatorname{soc} Y$.

(d) First of all, thanks to (a) and Lemma 5.6(b), B-cotop induces a surjection from $\mathcal{O}$ to ind $(\operatorname{soc} U)$. Let us prove that it is injective. Suppose that $P, P^{\prime} \in \mathcal{O}$ satisfy $S:=B$-cotop $P=B$-cotop $P^{\prime}$ and consider the short exact sequences

$$
0 \rightarrow P \stackrel{f}{\rightarrow} B \text {-corad } P \stackrel{g}{\rightarrow} S \rightarrow 0 \quad \text { and } \quad 0 \rightarrow P^{\prime} \stackrel{f^{\prime}}{\rightarrow} B \text {-corad } P^{\prime} \stackrel{g^{\prime}}{\rightarrow} S \rightarrow 0 .
$$

Multiplying them by $e$, we get $B$-corad $P, B$-corad $P^{\prime} \in \mathrm{CM}_{e} A$. So, applying $\operatorname{Hom}_{A}(B-\operatorname{corad} P,-)$ to the second short exact sequence, we get a morphism $u: B$-corad $P \rightarrow B$-corad $P^{\prime}$ such that $g=u g^{\prime}$. Symmetrically, we get a morphism $u^{\prime}: B$-corad $P^{\prime} \rightarrow B$-corad $P$ such that $g^{\prime}=u^{\prime} g$. So $g=u u^{\prime} g$ and, as $g$ is right minimal, $u u^{\prime}$ is an isomorphism. Similarly, $u^{\prime} u$ is an isomorphism so $B$-corad $P \cong$ $B$-corad $P^{\prime}$ and $P \cong P^{\prime}$. We proved that $B$-cotop is injective on $\mathcal{O}$.

That $\operatorname{Hom}_{A}\left(B,-\otimes_{R}(K / R)\right): \mathcal{O} \rightarrow$ add $U$ is well-defined is a direct consequence of the definition of $U$. The commutativity of the diagram is immediate by Lemma 5.2(a). As $U$ is injective, $\operatorname{soc}:$ ind $U \rightarrow$ ind ( $\operatorname{soc} U)$ is bijective.

The following proposition is used to categorify cluster algebras in Section 6 .

Proposition 5.9. If (E1) is satisfied, then the following assertions hold:

(a) If $X \in \mathrm{CM}_{e} A$ does not have nonzero direct summands in add Ae, then $T X \in$ add $\mathcal{O}$. Moreover, $B$-corad $T X \subset X$ and $B$-cotop $T X \cong \operatorname{soc} F X$.

(b) Let $0 \rightarrow X \rightarrow Y \rightarrow Z \rightarrow 0$ be a short exact sequence with $X, Z \in \mathrm{CM}_{e} A$ without nonzero direct summands in add Ae. Then the maximal direct summand $Y_{1}$ of $Y$ in add Ae is the module satisfying $Y_{1} \in \operatorname{add} \mathcal{O}$ and $\operatorname{soc} F X \oplus \operatorname{soc} F Z \cong$ $\operatorname{soc} F Y \oplus B$-cotop $Y_{1}$.

Proof. (a) Since $T X \rightarrow X$ is radical, the result follows from Lemma 5.8(c).

(b) Decompose $Y=Y_{0} \oplus Y_{1}$. Recall that $T=A e \otimes_{e A e} e-$ is exact on $\bmod _{e} A$. As $T X$ is projective, we get

$$
T Y_{0} \oplus Y_{1}=T Y \cong T X \oplus T Z \in \operatorname{add} \mathcal{O}
$$

by (a). Again by (a), we get

$\operatorname{soc} F X \oplus \operatorname{soc} F Z \cong B$-cotop $T X \oplus B$-cotop $T Z \cong B$-cotop $T Y_{0} \oplus B$-cotop $Y_{1}$

$$
\cong \operatorname{soc} F Y_{0} \oplus B \text {-cotop } Y_{1} \cong \operatorname{soc} F Y \oplus B \text {-cotop } Y_{1} \text {. }
$$

5B. Proof of Theorem 2.1. (a) Since $A e \in$ add $\operatorname{Hom}_{R}(g A, R)$, the conditions (E1) and (E2) are satisfied by Lemma 5.7 and cotop $A e=$ top $A g$ by Lemma 5.2(c). By Theorem 2.2, we have an equivalence of exact categories

$$
B \otimes_{A}-:\left(\mathrm{CM}_{e} A\right) /[A e] \cong \operatorname{Sub} U
$$


and $U$ is an injective $B$-module. Thanks to Lemma $5.6(\mathrm{~b})$, we have $\operatorname{soc} U \cong$ $B$-cotop $A e \cong \operatorname{Hom}_{A}(B$, top $A g)$. Thus $U \cong Q_{g}$.

(b) For $M \in \operatorname{Sub} Q_{g}$, let us consider a projective cover of $D_{0} M$ in $\bmod A^{\mathrm{op}}$ :

$$
0 \rightarrow \Omega_{A} D_{0} M \rightarrow P \rightarrow D_{0} M \rightarrow 0 .
$$

We have $P \in \operatorname{add} g A$. Applying $\operatorname{Hom}_{R}(-, R)$, we get the short exact sequence

$$
0 \rightarrow D_{1} P \rightarrow D_{1} \Omega_{A} D_{0} M \rightarrow M \rightarrow 0 .
$$

We have $D_{1} P \in$ add $A e$ so $D_{1} \Omega_{A} D_{0} M \in \mathrm{CM}_{e} A$ and $F\left(D_{1} \Omega_{A} D_{0} M\right) \cong M$ thanks to this sequence.

(c) Let us assume first that $A e, A f$ and $A g$ are basic. In particular $A e \cong D_{1}(g A)$, $A f \cong D_{1}(e A)$ as $A$-modules and $e A e \cong D_{1}(e A e)$ as left $(e A e)$-modules. We have $e A f \cong e D_{1}(e A)=D_{1}(e A e) \cong e A e$ as left $(e A e)$-modules. So $A f \in \mathrm{CM}_{e} A$ and $T(A f)=A e \otimes_{e A e} e A f \cong A e$. Moreover, using the short exact sequence $0 \rightarrow T(A f) \rightarrow A f \rightarrow F(A f) \rightarrow 0$ and Lemma 5.4, we get

$$
\operatorname{soc} B f=\operatorname{soc} F(A f) \subset B \text {-cotop } T(A f) \cong B \text {-cotop } A e \cong \text { top } B g,
$$

so $B f \subset D_{0}(g B)$. Dually, we get an inclusion $g B \subset D_{0}(B f)$ by exchanging the role of $f$ and $g$. By comparing lengths over $R$ of $g B$ and $B f$, we deduce that $B f \cong D_{0}(g B)=Q_{g}$.

If $A e, A f$ or $A g$ are not basic, we take basic parts $e^{\prime}, f^{\prime}$ and $g^{\prime}$ of $e, f$ and $g$ and we get $B f^{\prime} \cong Q_{g^{\prime}}$. Thus add $B f=$ add $B f^{\prime}=$ add $Q_{g^{\prime}}=$ add $Q_{g}$.

(d) Since $A \in \mathrm{CM}_{e} A$, we have $B=F A \in \operatorname{Sub} Q_{g}$. Thus Sub $Q_{g}=\operatorname{Sub} B$ holds by (c).

(e) All assumptions in Theorem 4.8 are satisfied. Moreover, since $A \in \mathcal{C}$, the projective objects in $\mathcal{E}=\bmod _{e} A$ and $\mathcal{C}=\mathrm{CM}_{e} A$ are projective $A$-modules, and the equivalent conditions of Theorem 4.8 (a) are satisfied. Thus applying Theorem 4.8(d) (i) $\Leftrightarrow$ (iii), $B$ is Iwanaga-Gorenstein of dimension at most 1 if and only if $\mathrm{CM}_{e} A$ is Frobenius. As $A$ and $D_{1} A$ are in $\mathrm{CM}_{e} A$, we get that $A$ is Gorenstein if and only if add $A=\operatorname{add} D_{1} A$ if and only if $\mathrm{CM}_{e} A$ is Frobenius, and the result follows.

(f) In this case, $\left(\mathrm{CM}_{e} A\right) /[A e] \cong \mathrm{Sub} B$ is an equivalence of Frobenius categories. Thus, since $\underline{\mathrm{CM}}_{e} A$ coincides with the stable category of $\left(\mathrm{CM}_{e} A\right) /[A e]$, we have a triangle equivalence $\underline{\mathrm{CM}}_{e} A \cong \underline{\mathrm{Sub}} B$.

5C. Proof of Theorem 2.3. By construction, we have an exact sequence

$$
0 \rightarrow P_{W} \rightarrow W \rightarrow B \rightarrow 0
$$

with $W=A e \oplus \widetilde{B} \in \mathrm{CM} A$ and $P_{W}=A e \oplus P \in$ add $A e$. Clearly we have $W \in \mathrm{CM}_{e}^{B} A$ and $P_{W}=A e \otimes_{e A e} e W$. We set $A^{\prime}:=\operatorname{End}_{A}(W)$ and we identify $e$ with the idempotent 
of $A^{\prime}$ which is the projection on the summand $A e$ of $W$. Thus, we can identify $e A e$ and $e A^{\prime} e$. We shall prove (a) in Proposition 5.11, (b) in Proposition 5.15 and (d) in Proposition 5.12. Then all hypotheses of Theorem 2.2 are satisfied and the assertion (c) follows. Finally, (e) is an easy consequence of Proposition 5.12.

Lemma 5.10. (a) We have $W e=A e$ and $W(1-e)=\widetilde{B}$ as A-modules.

(b) We have $W e A^{\prime}=P_{W}$. Thus $P_{W}$ and $B$ have a structure of $A^{\prime \text { op }}$-modules such that (5-2) is an exact sequence of $\left(A, A^{\prime}\right)$-bimodules.

(c) We have $W / W e A^{\prime} \cong B$ as $\left(A, A^{\prime}\right)$-bimodules.

(d) We have $e A^{\prime}=e W$, and this is a projective ( $e A e$ )-module and a projective $A^{\prime \mathrm{op}_{-} \text {module. }}$.

(e) We have $B \otimes_{A} W \cong B$ as $\left(B, A^{\prime}\right)$-bimodules.

Proof. (a) This is clear from the definition.

(b) Since $W e=A e$, we have $W e A^{\prime}=\sum_{f \in \operatorname{End}_{A}(W)} f(A e)=A e \otimes_{e A e} e W=P_{W}$. The map $P_{W} \rightarrow W$ is clearly a morphism of $\left(A, A^{\prime}\right)$-bimodules.

(c) This is a clear consequence of (b).

(d) We have $e A^{\prime}=\operatorname{Hom}_{A}(A e, W)=e W$. Clearly $e A^{\prime}$ is a projective $A^{\prime \text { op }}$-module. Moreover $e W=e P_{W}$ is a projective $(e A e)$-module since $P_{W} \in$ add $A e$.

(e) Applying $B \otimes_{A}-$ to the short exact sequence (5-2), we get the exact sequence of $\left(B, A^{\prime}\right)$-bimodules

$$
B \otimes_{A} P_{W} \rightarrow B \otimes_{A} W \rightarrow B \otimes_{A} B \rightarrow 0 .
$$

Since $B \otimes_{A} P_{W} \in \operatorname{add}\left(B \otimes_{A} A e\right)=\operatorname{add}(B e)=\{0\}$ and $B \otimes_{A} B \cong B$, we get the result.

Proposition 5.11. We have an isomorphism $A^{\prime} /(e) \cong B$ of $R$-algebras and an isomorphism $W \otimes_{A^{\prime}} B \cong B$ of $(A, B)$-bimodules.

Proof. Applying $\operatorname{Hom}_{A}(W,-)$ to (5-2), we have an exact sequence

$$
0 \rightarrow \operatorname{Hom}_{A}\left(W, P_{W}\right) \rightarrow A^{\prime} \rightarrow \operatorname{Hom}_{A}(W, B) \rightarrow \operatorname{Ext}_{A}^{1}\left(W, P_{W}\right),
$$

where $\operatorname{Ext}_{A}^{1}\left(W, P_{W}\right)=0$ by $P_{W} \in \operatorname{add} A e, W \in \mathrm{CM}_{e}^{B} A$ and our assumption $\operatorname{Ext}_{A}^{1}\left(\mathrm{CM}_{e}^{B}, A e\right)=0$. Since $\operatorname{Hom}_{A}(A e, B)=0$, applying $\operatorname{Hom}_{A}(-, B)$ to (5-2), we have $\operatorname{Hom}_{A}(W, B)=\operatorname{End}_{A}(B)=B$ and $(e)=\operatorname{Hom}_{A}\left(W, P_{W}\right)$. Thus $A^{\prime} /(e)=$ $A^{\prime} / \operatorname{Hom}_{A}\left(W, P_{W}\right)=\operatorname{Hom}_{A}(W, B)=B$.

We have $W \otimes_{A^{\prime}} B=W / W e A^{\prime}=B$ by Lemma 5.10(c).

In particular, we can regard $\bmod B$ as full subcategory of both $\bmod A^{\prime}$ and $\bmod A$. Now we consider the adjoint pair $(G, H)$ given by

$$
H:=\operatorname{Hom}_{A}(W,-): \bmod A \rightarrow \bmod A^{\prime} \quad \text { and } \quad G:=W \otimes_{A^{\prime}}-: \bmod A^{\prime} \rightarrow \bmod A .
$$


The main result about these functors is:

Proposition 5.12. The class of short exact sequences of $\bmod A$ with three terms in $\bmod _{e}^{B} A$ gives the structure of an exact category on $\bmod _{e}^{B} A$. The same holds for $\mathrm{CM}_{e}^{B} A$. For these structures, the adjoint pair $(G, H)$ gives quasi-inverse equivalences of exact categories between $\bmod _{e}^{B} A$ and $\bmod _{e} A^{\prime}$, which restrict to quasi-inverse equivalences of exact categories between $\mathrm{CM}_{e}^{B} A$ and $\mathrm{CM}_{e} A^{\prime}$.

The first step of the proof consists of the following lemma.

Lemma 5.13. (a) $H$ and $G$ give quasi-inverse equivalences between add $A e$ and add $A^{\prime} e$.

(b) We have commutative diagrams
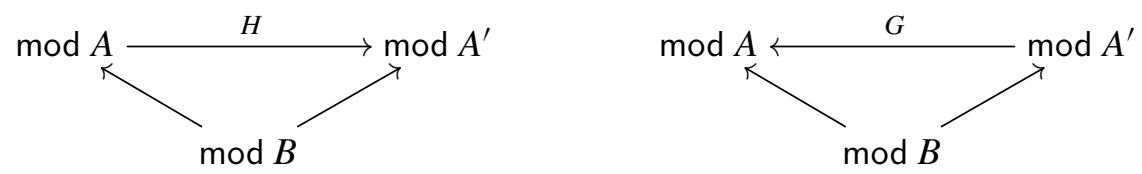

Proof. (a) This is clear: $H(A e)=\operatorname{Hom}_{A}(W, A e)=A^{\prime} e$ and $G\left(A^{\prime} e\right) \cong W e=A e$ by Lemma $5.10($ a).

(b) Fix $X \in \bmod B$. Applying $\operatorname{Hom}_{A}(-, X)$ to (5-2), we have an exact sequence

$$
0 \rightarrow \operatorname{Hom}_{A}(B, X) \rightarrow H X \rightarrow \operatorname{Hom}_{A}\left(P_{W}, X\right),
$$

where $\operatorname{Hom}_{A}\left(P_{W}, X\right)=0$ by $P_{W} \in \operatorname{add} A e$ and $X \in \bmod B$. Thus we have

$$
H X \cong \operatorname{Hom}_{A}(B, X) \cong X .
$$

On the other hand, we have

$$
G(X)=W \otimes_{A^{\prime}} X=W \otimes_{A^{\prime}}\left(B \otimes_{A^{\prime}} X\right) \stackrel{\text { Proposition }}{=} 5.11
$$

Lemma 5.14. (a) We have $\operatorname{Tor}_{1}^{A^{\prime}}(Y, X)=\operatorname{Tor}_{1}^{B}\left(Y \otimes_{A^{\prime}} B, X\right)$ for any $X \in \bmod B$ and $Y \in \mathrm{CM} A^{\prime \mathrm{op}}$.

(b) We have $\operatorname{Tor}_{1}^{A^{\prime}}(W, X)=0$ for any $X \in \bmod _{e} A^{\prime}$.

Proof. For $Y \in \mathrm{CM} A^{\prime \text { op }}$, take an exact sequence

$$
0 \rightarrow \Omega Y \stackrel{i}{\rightarrow} P \rightarrow Y \rightarrow 0
$$

of $A^{\prime \text { op }}$-modules with $P \in \operatorname{proj} A^{\prime \text { op }}$. We will show that

$$
0 \rightarrow \Omega Y \otimes_{A^{\prime}} B \stackrel{i \otimes 1_{B}}{\longrightarrow} P \otimes_{A^{\prime}} B \rightarrow Y \otimes_{A^{\prime}} B \rightarrow 0
$$

is exact. Consider the exact sequence

$$
A^{\prime} e \otimes_{e A^{\prime} e} e A^{\prime} \stackrel{j}{\longrightarrow} A^{\prime} \rightarrow B \rightarrow 0
$$

of $\left(A^{\prime}, A^{\prime}\right)$-bimodules. Applying $Y \otimes_{A^{\prime}}-$, we have an exact sequence

$$
0 \rightarrow K \rightarrow Y e \otimes_{e A^{\prime} e} e A^{\prime} \stackrel{1_{Y} \otimes j}{\longrightarrow} Y \rightarrow Y \otimes_{A^{\prime}} B \rightarrow 0 .
$$


Since $\left(1_{Y} \otimes j\right) e:\left(Y e \otimes_{e A^{\prime} e} e A^{\prime}\right) e \rightarrow Y e$ is an isomorphism, we have $K e=0$. Thus $K$ is a $B^{\mathrm{op}}$-module. Since $e A^{\prime} \in \operatorname{proj}\left(e A^{\prime} e\right)$ by Lemma 5.10(d), we get $Y e \otimes_{e A^{\prime} e} e A^{\prime} \in \mathrm{CM} A^{\prime \text { op }}$. Therefore $K=0$.

Applying the same argument to $P \in \mathrm{CM} A^{\prime \text { op }}$ and $\Omega Y \in \mathrm{CM} A^{\prime \text { op }}$, we have the following commutative diagram of exact sequences:

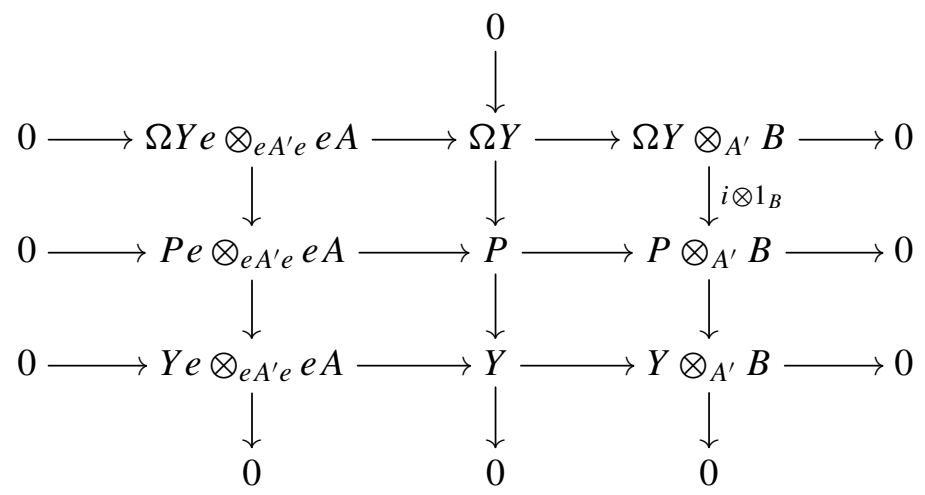

By the snake lemma, $i \otimes 1_{B}$ is injective. Thus (5-4) is exact.

(a) For $X \in \bmod B$, applying $-\otimes_{A^{\prime}} X$ to (5-3) and $-\otimes_{B} X$ to (5-4) and comparing them, we have a commutative diagram of exact sequences:

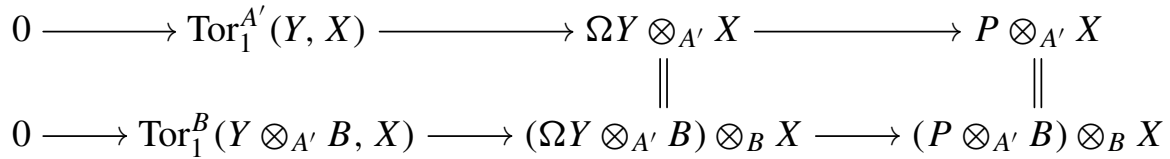

Thus the assertion follows.

(b) First, we assume $X \in \bmod B$. Since $W \in C M A^{\prime \text { op }}$, by (a) and Proposition 5.11, we have

$$
\operatorname{Tor}_{1}^{A^{\prime}}(W, X) \cong \operatorname{Tor}_{1}^{B}\left(W \otimes_{A^{\prime}} B, X\right) \cong \operatorname{Tor}_{1}^{B}(B, X)=0 .
$$

Now we assume $X \in \bmod _{e} A^{\prime}$. Then there exists an exact sequence $0 \rightarrow P \rightarrow X \rightarrow$ $Y \rightarrow 0$ with $P \in$ add $A^{\prime} e$ and $Y \in \bmod B$. Applying $W \otimes_{A^{\prime}}-$, we have an exact sequence

$$
0=\operatorname{Tor}_{1}^{A^{\prime}}(W, P) \rightarrow \operatorname{Tor}_{1}^{A^{\prime}}(W, X) \rightarrow \operatorname{Tor}_{1}^{A^{\prime}}(W, Y)=0 .
$$

Thus the assertion follows.

Proof of Proposition 5.12. (i) First we show $H\left(\bmod _{e} A\right) \subset \bmod _{e} A^{\prime}$.

For $X \in \bmod _{e} A$, we get, using Lemma 5.10(a),

$$
e H(X)=\operatorname{Hom}_{A}(W e, X)=\operatorname{Hom}_{A}(A e, X)=e X \in \operatorname{proj}(e A e)=\operatorname{proj}\left(e A^{\prime} e\right) .
$$

(ii) Next we show $G\left(\bmod _{e} A^{\prime}\right) \in \bmod _{e}^{B} A$. 
For $X \in \bmod _{e} A^{\prime}$, take an exact sequence

$$
0 \rightarrow P \rightarrow X \rightarrow Y \rightarrow 0
$$

with $P \in$ add $A^{\prime} e$ and $Y \in \bmod B$. Applying $G$, we have a short exact sequence

$$
0 \rightarrow G P \rightarrow G X \rightarrow G Y \rightarrow 0
$$

by Lemma 5.14(b). Since $G P \in$ add $A e$ and $G Y=Y \in \bmod B$ thanks to Lemma 5.13, we have $G X \in \bmod _{e}^{B} A^{\prime}$.

(iii) We now show $H G \cong \mathrm{id}_{\bmod _{e} A^{\prime}}$ and $G H \cong \operatorname{id}_{\bmod _{e}^{B} A}$.

Applying $H$ to (5-6) and comparing with (5-5), we have a commutative diagram of exact sequences

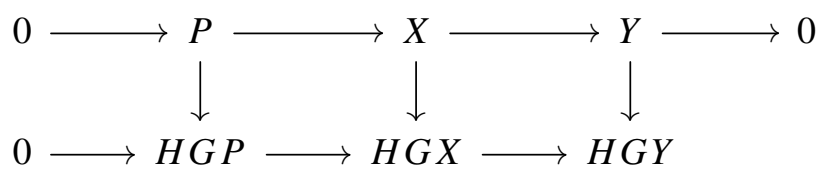

where vertical arrows are of the form $x \mapsto(w \mapsto w \otimes x)$. Since the left and the right vertical maps are isomorphisms, so is the middle one.

By a similar argument, one can show $G H \cong \mathrm{id}_{\bmod _{e}^{B} A}$.

(iv) Next we show that $H: \bmod _{e}^{B} A \rightarrow \bmod _{e} A^{\prime}$ and $G: \bmod _{e} A^{\prime} \rightarrow \bmod _{e}^{B} A$ preserve short exact sequences. In particular, $\bmod _{e}^{B} A$ has the desired exact structure.

The functor $G$ is exact thanks to Lemma 5.14(b). Consider a short exact sequence $0 \rightarrow X \rightarrow Y \rightarrow Z \rightarrow 0$ in $\bmod A$ with three terms in $\bmod _{e}^{B} A$. We get an exact sequence $0 \rightarrow H X \rightarrow H Y \rightarrow H Z \rightarrow C \rightarrow 0$ with $C \in \bmod A^{\prime}$. As $G$ is right exact and by (iii), we deduce $W \otimes_{A^{\prime}} C=G C=0$, so $e C=e W \otimes_{A^{\prime}} C=0$ by Lemma 5.10(d), so $C \in \bmod B$. Hence by Lemma 5.13(b), $C=0$ so $0 \rightarrow H X \rightarrow H Y \rightarrow H Z \rightarrow 0$ is exact in $\bmod _{e} A^{\prime}$.

(v) We now show that the equivalences restrict to $\mathrm{CM}_{e}^{B} A \cong \mathrm{CM}_{e} A^{\prime}$.

Clearly $H\left(\mathrm{CM}_{e}^{B} A\right) \subset \mathrm{CM}_{e} A^{\prime}$ holds. It is enough to show that, if $X \in \bmod _{e}^{B} A$ satisfies $H X \in \mathrm{CM}_{e} A^{\prime}$, then $X \in \mathrm{CM} A$. Let $Y$ be a finite-length submodule of $X$. Then the inclusion $Y \subset X$ gives an injection $H Y \subset H X$. Since $H Y$ has finite length and $H X \in \mathrm{CM}_{e} A^{\prime}$, we have $H Y=0$.

Let $0 \rightarrow P \stackrel{i}{\longrightarrow} X \rightarrow Z \rightarrow 0$ be an exact sequence with $P \in \operatorname{add} A e$ and $Z \in \bmod B$. Since $Y \cap P=0$, we have that $Y$ is a submodule of $Z$. In particular $Y \in \bmod B$. Since $H Y=0$, we have $Y=0$ by (iii). Thus $X \in \mathrm{CM} A$.

Proposition 5.15. We have (E1), that is, $\operatorname{Ext}_{A^{\prime}}^{1}\left(\mathrm{CM}_{e} A^{\prime}, A^{\prime} e\right)=0$, and $(\mathrm{E} 2)^{+}$, that is, $\operatorname{Ext}_{A^{\prime}}^{2}\left(\bmod _{e} A^{\prime}, A^{\prime} e\right)=0$.

Proof. (E1): Let $0 \rightarrow A^{\prime} e \rightarrow X \rightarrow Y \rightarrow 0$ be an exact sequence with $Y \in \mathrm{CM}_{e} A^{\prime}$. Applying $G$ and using Lemma 5.14(b), we have an exact sequence $0 \rightarrow G\left(A^{\prime} e\right) \rightarrow$ 
$G X \rightarrow G Y \rightarrow 0$. It splits since $\operatorname{Ext}_{A}^{1}(G Y, A e)=0$ by our assumption. Since $G: \mathrm{CM}_{e} A^{\prime} \rightarrow \mathrm{CM}_{e}^{B} A$ is an equivalence, the original sequence splits. Thus the assertion follows.

$(\mathrm{E} 2)^{+}$: Since we have $A^{\prime} \in \mathrm{CM}_{e} A^{\prime}$ by Lemma 5.10(d), syzygies of modules in $\bmod _{e} A^{\prime}$ belong to $\mathrm{CM}_{e} A^{\prime}$. Thus the assertion follows from (E1).

We finish this subsection by proving Lemma 2.4.

Proof of Lemma 2.4. As $A e \cong D_{1}(g A)$ is injective in $\mathrm{CM} A$ and $\mathrm{CM}_{e}^{B} A \subset \mathrm{CM} A$, we get (C3).

To prove the second part of the statement, let us prove that if $(\mathrm{C} 1)$ holds, then for a finite-length $A$-module $M$, we have $M \in \operatorname{Sub}\left(A e \otimes_{R}(K / R)\right)$ if and only if $(1-g) \operatorname{soc} M=0$. As $A e$ is injective in $\mathrm{CM} A$ and syzygies of all modules are Cohen-Macaulay, we have $\operatorname{Ext}_{A}^{2}(\bmod A, A e)=0$. By Lemma 5.1, we have $\operatorname{Ext}_{A}^{1}\left(\right.$ f.I. $\left.A, A e \otimes_{R} K\right)=0$. So applying $\operatorname{Hom}_{A}($ f.I. $A,-)$ to the short exact sequence

$$
0 \rightarrow A e \rightarrow A e \otimes_{R} K \rightarrow A e \otimes_{R}(K / R) \rightarrow 0,
$$

we get $\operatorname{Ext}_{A}^{1}$ (f.I. $\left.A, A e \otimes_{R}(K / R)\right)=0$. Moreover, by Lemma 5.2(a), we get that $\operatorname{soc}\left(A e \otimes_{R}(K / R)\right)$ is the semisimple module corresponding to $g$.

If $M \in \operatorname{Sub}\left(A e \otimes_{R}(K / R)\right)$ then $\operatorname{soc} M \in$ add $\operatorname{soc}\left(A e \otimes_{R}(K / R)\right)$ follows immediately, and thus the first implication is satisfied. Conversely, if $(1-g) \operatorname{soc} M=0$, then there exists an injection $\operatorname{soc} M \hookrightarrow\left(A e \otimes_{R}(K / R)\right)^{\oplus \ell}$. Then, by applying $\operatorname{Hom}_{A}\left(-,\left(A e \otimes_{R}(K / R)\right)^{\oplus \ell}\right)$ to the short exact sequence

$$
0 \rightarrow \operatorname{soc} M \rightarrow M \rightarrow M / \operatorname{soc} M \rightarrow 0
$$

and using $\operatorname{Ext}_{A}^{1}\left(M / \operatorname{soc} M, A e \otimes_{R}(K / R)\right)=0$, there is an injection $M \hookrightarrow\left(A e \otimes_{R}\right.$ $(K / R))^{\oplus \ell}$, and so we have proved the converse implication.

\section{Cluster algebra structure on coordinate rings of partial flag varieties}

The aim of this section is to apply results in previous sections to categorify the cluster algebra structure of the multihomogeneous coordinate rings $\mathbb{C}[\mathcal{F}]$ of the partial flag variety $\mathcal{F}=\mathcal{F}(\Delta, J)$ corresponding to a Dynkin diagram $\Delta$ and a set $J$ of vertices of $\Delta$ by using the category of Cohen-Macaulay modules. To be more precise, recall that Geiss, Leclerc and Schröer [2008] introduced a cluster algebra $\tilde{\mathcal{A}} \subset \mathbb{C}[\mathcal{F}]$. They proved that $\tilde{\mathcal{A}}=\mathbb{C}[\mathcal{F}]$ in type $A_{n}$. In general, they conjecture that $\tilde{\mathcal{A}}\left[\Sigma_{J}^{-1}\right]=\mathbb{C}[\mathcal{F}]\left[\Sigma_{J}^{-1}\right]$, where $\Sigma_{J}$ is the set of principal generalized minors corresponding to nonminuscule weights (see Definition 6.3 of principal generalized minors), and they prove the conjecture in type $D_{4}$.

The main result of this section (Theorem 6.12) consists of completing Geiss, Leclerc and Schröer's partial categorification of $\tilde{\mathcal{A}}$. Their categorification, given in Theorem 6.6, uses the preprojective algebra $\Pi=\Pi(\Delta)$ over $\mathbb{C}$ and the full 
subcategory Sub $Q_{J}$ of $\bmod \Pi$, where $Q_{J}$ is the direct sum of indecomposable injective $\Pi$-modules corresponding to vertices in $J$. Recall that a Frobenius category $\mathcal{E}$ is said to be stably 2-Calabi-Yau if there is a bifunctorial isomorphism $\operatorname{Ext}_{\mathcal{E}}^{1}(X, Y) \cong D \operatorname{Ext}_{\mathcal{E}}^{1}(Y, X)$ and that Sub $Q_{J}$ is stably 2-Calabi-Yau. Moreover, an object $X$ in $\mathcal{E}$ is called rigid if $\operatorname{Ext}_{\mathcal{E}}^{1}(X, X)=0$ and it is called cluster tilting if add $X=\left\{Y \in \mathcal{E} \mid \operatorname{Ext}_{\mathcal{E}}^{1}(Y, X)=0\right\}$.

6A. The categorification of Geiss, Leclerc and Schröer. We recall briefly the results of [Geiss et al. 2008] concerning the categorification of cluster algebra structures on multihomogeneous coordinate rings of partial flag varieties. We start by fixing a simple simply connected complex algebraic group $G$ with Dynkin diagram $\triangle$. We fix a maximal torus $H \subset G$ and two opposite Borel subgroups $B, B^{-} \subset G$ satisfying $B \cap B^{-}=H$ (for more details about Lie theoretical background, see [Borel 1991; Lakshmibai and Gonciulea 2001]). For a vertex $i$ of $\Delta$, we fix

$$
x_{i}(t):=\exp \left(t e_{i}\right) \quad \text { and } \quad y_{i}(t):=\exp \left(t f_{i}\right),
$$

the one-parameter subgroups of $B$ and $B^{-}$corresponding to the Chevalley generators $e_{i}$ and $f_{i}$ of the Lie algebra of $G$. Following notations of [Geiss et al. 2008], we define $K$ to be the complement of $J$. The parabolic subgroup $B_{K}$ of $G$ is the subgroup generated by $B$ and $y_{i}$ for $i \in K$, and the opposite parabolic subgroup $B_{K}^{-}$of $G$ is the subgroup generated by $B^{-}$and $x_{i}$ for $i \in K$. The partial flag variety $\mathcal{F}$ can be realized as $\mathcal{F}=B_{K}^{-} \backslash G$. Let $N_{K}$ be the unipotent radical of $B_{K}$, that is, the subgroup of unipotent elements of the maximal solvable normal subgroup of $B_{K}$. Then, it is a classical result that $N_{K} \subset G$ induces an embedding $N_{K} \subset \mathcal{F}$ as a dense affine open subset.

Example 6.1. If $\Delta=A_{4}$ and $J=\{1,3\}$, we have $K=\{2,4\}, G=\mathrm{SL}_{5}(\mathbb{C})$ and

$$
B_{K}^{-}=\left[\begin{array}{ccccc}
* & 0 & 0 & 0 & 0 \\
* & * & * & 0 & 0 \\
* & * & * & 0 & 0 \\
* & * & * & * & * \\
* & * & * & * & *
\end{array}\right] \subset G \text { and } N_{K}=\left[\begin{array}{ccccc}
1 & * & * & * & * \\
0 & 1 & 0 & * & * \\
0 & 0 & 1 & * & * \\
0 & 0 & 0 & 1 & 0 \\
0 & 0 & 0 & 0 & 1
\end{array}\right],
$$

and it is immediate that $B_{K}^{-} \backslash G$ parametrizes naturally flags of $\mathbb{C}^{5}$ of type $(1,3)$.

Let $\boldsymbol{i}=\left(i_{1}, i_{2}, \ldots, i_{\ell}\right)$ be a sequence of vertices of $\Delta$, let $\boldsymbol{k}=\left(k_{1}, k_{2}, \ldots, k_{\ell}\right)$ be a sequence of nonnegative integers and let $t=\left(t_{1}, t_{2}, \ldots, t_{\ell}\right)$ be a sequence of variables. We define

- $\boldsymbol{i}^{k}$, the sequence of indices obtained from $\boldsymbol{i}$ by repeating $k_{j}$ times $i_{j}$;

- $\boldsymbol{t}^{\boldsymbol{k}}:=t_{1}^{k_{1}} t_{2}^{k_{2}} \cdots t_{\ell}^{k_{\ell}}$;

- $\boldsymbol{k} !:=k_{1} ! k_{2} ! \cdots k_{\ell} !$

- $x_{i}(\boldsymbol{t}):=x_{i_{1}}\left(t_{1}\right) x_{i_{2}}\left(t_{2}\right) \cdots x_{i_{\ell}}\left(t_{\ell}\right)$. 
For a vertex $i$ of $\Delta$, we denote by $S_{i}$ the simple $\Pi$-module corresponding to $i$. Then, for $M \in \bmod \Pi$, we denote by $\Phi_{M, i}$ the variety of composition series of $M$ of type $i$, that is,

$$
\Phi_{M, i}:=\left\{0=M_{0} \subset M_{1} \subset \cdots \subset M_{\ell}=M \mid \forall j, M_{j} / M_{j-1} \cong S_{i_{j}}\right\},
$$

realized within the appropriate product of Grassmannians. Finally $\chi$ is the Euler characteristic.

Using Lusztig's semicanonical basis [2000], Geiss, Leclerc and Schröer [Geiss et al. 2005] define functions in the coordinate ring $\mathbb{C}[N]=\mathbb{C}\left[N_{\varnothing}\right]$ by the following result:

Theorem 6.2 ([Lusztig 2000; Geiss et al. 2005]). Let $M \in \bmod \Pi$. There exists a unique function $\varphi_{M}$ in $\mathbb{C}[N]$ satisfying

$$
\varphi_{M}\left(x_{i}(t)\right)=\sum_{\boldsymbol{k} \in \mathbb{N}^{\ell}} \chi\left(\Phi_{M, i^{k}}\right) \frac{\boldsymbol{t}^{\boldsymbol{k}}}{\boldsymbol{k} !}
$$

for any reduced word $\boldsymbol{i}$ of an element of the Weyl group of type $\Delta$.

In [Geiss et al. 2005], they also prove that

- $\varphi_{Y \oplus Z}=\varphi_{Y} \varphi_{Z}$ for any $Y, Z \in \bmod \Pi$;

- if $Y$ and $Z$ are indecomposable such that $\operatorname{dim} \operatorname{Ext}_{\Pi}^{1}(Y, Z)=1$ and

$$
0 \rightarrow Y \rightarrow U \rightarrow Z \rightarrow 0 \text { and } 0 \rightarrow Z \rightarrow U^{\prime} \rightarrow Y \rightarrow 0
$$

are two nonsplit short exact sequences, then $\varphi_{Y} \varphi_{Z}=\varphi_{U}+\varphi_{U^{\prime}}$.

In other terms, $\varphi$ is a so-called cluster character.

In [Geiss et al. 2008], the authors prove that Sub $Q_{J}$ categorifies via $\varphi$ and the canonical projection $\mathbb{C}[N] \rightarrow \mathbb{C}\left[N_{K}\right]$ a cluster algebra $\mathcal{A} \subset \mathbb{C}\left[N_{K}\right]$. They prove in type $A_{n}$ and $D_{4}$ that $\mathcal{A}=\mathbb{C}\left[N_{K}\right]$ and they conjecture it to be true in any case.

Let us introduced generalized principal minors (see [Fomin and Zelevinsky 1999]):

Definition 6.3. For a vertex $i$ of $\Delta$, the corresponding principal generalized minor is defined on $G$ as the unique function $\Delta_{i}$ satisfying

$$
\Delta_{i}\left(x^{-} x_{0} x^{+}\right)=\Delta_{i}\left(x_{0}\right)
$$

for $x^{-} \in B^{-}, x_{0} \in H$ and $x^{+} \in B$, and $\left.\Delta_{i}\right|_{H}: H \rightarrow \mathbb{C}^{*}$ is the multiplicative character corresponding to the fundamental weight indexed by $i$.

It is known that $\mathcal{F}=B_{K}^{-} \backslash G$ is embedded in a product of projective spaces indexed by $J$ (in type $A_{n}$, a product of usual Grassmannians). Thus, we can define the multihomogeneous coordinate ring $\mathbb{C}[\mathcal{F}]$, graded by $\mathbb{N}^{J}$. Each of the $\Delta_{j}$ is homogeneous of degree $(0, \ldots, 0,1,0, \ldots, 0)$, where 1 is at position $j$ and $N_{K}$ is 
the open dense affine subset of $\mathcal{F}$ defined by $N_{K}=\left\{x \in \mathcal{F} \mid \forall j \in J, \Delta_{j}(x) \neq 0\right\}$, so there is a dehomogenization map $\mathbb{C}[\mathcal{F}] \rightarrow \mathbb{C}\left[N_{K}\right]$ defined by mapping $\Delta_{j}$ to 1 . For any $f \in \mathbb{C}\left[N_{K}\right]$, there is a unique homogeneous $\tilde{f} \in \mathbb{C}[\mathcal{F}]$ such that $\pi(\tilde{f})=f$ and the multidegree of $\tilde{f}$ is minimal for the order induced by fundamental weights [Geiss et al. 2008, Lemma 2.4].

Example 6.4. We continue Example 6.1. In this case, $\Delta_{1}$ corresponds to the upper-left coefficient and $\Delta_{3}$ corresponds to the determinant of the upper-left $(3 \times 3)$-submatrix. Then $B_{K}^{-} \backslash G$ is a closed subset of $\mathrm{Gr}_{1}\left(\mathbb{C}^{5}\right) \times \mathrm{Gr}_{3}\left(\mathbb{C}^{5}\right)$, by mapping $M \in B_{K}^{-}$to the subspaces generated by the first row on the one hand and the first three rows on the second hand. So, as usual, thanks to Plücker coordinates, we have

$$
\mathcal{F} \subset \mathrm{Gr}_{1}\left(\mathbb{C}^{5}\right) \times \mathrm{Gr}_{3}\left(\mathbb{C}^{5}\right) \subset \mathbb{P}\left(\mathbb{C}^{\left(\begin{array}{l}
5 \\
1
\end{array}\right)}\right) \times \mathbb{P}\left(\mathbb{C}^{\left(\begin{array}{l}
5 \\
3
\end{array}\right)}\right) \text {. }
$$

Then, we have two affine subspaces $N_{\{1\}^{c}}$ of $\mathrm{Gr}_{1}\left(\mathbb{C}^{5}\right)$ and $N_{\{3\}^{c}}$ of $\mathrm{Gr}_{3}\left(\mathbb{C}^{5}\right)$ defined by the nonvanishing of the leftmost determinants, which are Plücker coordinates and correspond to $\Delta_{1}$ and $\Delta_{3}$ as functions over $G$. Moreover, $N_{K}=\left(N_{\{1\}^{c}} \times N_{\{3\}^{c}}\right) \cap \mathcal{F}$.

In order to extend the cluster algebra $\mathcal{A} \subset \mathbb{C}\left[N_{K}\right]$ to a cluster algebra $\tilde{\mathcal{A}} \subset \mathbb{C}[\mathcal{F}]$ by adding coefficients $\Delta_{j}$ corresponding to the multihomogenization, Geiss, Leclerc and Schröer prove the following theorem.

Theorem 6.5 [Geiss et al. 2008, 10.1]. If $Y, Z \in \operatorname{Sub} Q_{J}$, then $\tilde{\varphi}_{Y \oplus Z}=\tilde{\varphi}_{Y} \tilde{\varphi}_{Z}$. If $Y, Z \in \operatorname{Sub} Q_{J}$ satisfy $\operatorname{dim} \operatorname{Ext}_{\Pi}^{1}(Y, Z)=1$, and

$$
0 \rightarrow Y \rightarrow U \rightarrow Z \rightarrow 0 \text { and } 0 \rightarrow Z \rightarrow U^{\prime} \rightarrow Y \rightarrow 0
$$

are nonsplit short exact sequences, then

$$
\tilde{\varphi}_{Y} \tilde{\varphi}_{Z}=\tilde{\varphi}_{U} \prod_{j \in J} \Delta_{j}^{\alpha_{j}}+\tilde{\varphi}_{U^{\prime}} \prod_{j \in J} \Delta_{j}^{\beta_{j}}
$$

where

$$
\begin{aligned}
\alpha_{j} & =\max \left(0, \operatorname{dim} \operatorname{Hom}_{\Pi}\left(S_{j}, U^{\prime}\right)-\operatorname{dim} \operatorname{Hom}_{\Pi}\left(S_{j}, U\right)\right), \\
\beta_{j} & =\max \left(0, \operatorname{dim} \operatorname{Hom}_{\Pi}\left(S_{j}, U\right)-\operatorname{dim} \operatorname{Hom}_{\Pi}\left(S_{j}, U^{\prime}\right)\right) .
\end{aligned}
$$

To construct $\tilde{\mathcal{A}}$ using Theorem 6.5, Geiss, Leclerc and Schröer constructed an explicit cluster tilting object in Sub $Q_{J}$ that they call initial. A cluster tilting object in Sub $Q_{J}$ is called reachable if it is obtained from the initial one by successive mutations. An indecomposable rigid object is called reachable if it is a direct summand of a reachable cluster tilting object. Their result can be stated as follows.

Theorem 6.6 [Geiss et al. 2008, Theorem 10.2]. (a) There is a cluster algebra $\tilde{\mathcal{A}} \subset \mathbb{C}[\mathcal{F}]$ such that

- coefficients of $\tilde{\mathcal{A}}$ are $\tilde{c}$ for each coefficient c of $\mathcal{A}$ and $\Delta_{j}$ for each $j \in J$; 
- clusters of $\tilde{\mathcal{A}}$ are

$$
\left\{\tilde{x}_{1}, \tilde{x}_{2}, \ldots, \tilde{x}_{\ell}\right\} \sqcup\left\{\Delta_{j} \mid j \in J\right\}
$$

for each cluster $\left\{x_{1}, x_{2}, \ldots, x_{\ell}\right\}$ of $\mathcal{A}$.

(b) There is a bijection $X \mapsto \tilde{\varphi}_{X}$ between

- isomorphism classes of reachable indecomposable rigid objects of Sub $Q_{J}$;

- cluster variables and coefficients of $\tilde{\mathcal{A}}$ except $\Delta_{j}$ for $j \in J$.

(c) There is a bijection $\bigoplus_{k=1}^{\ell} T_{k} \mapsto\left\{\tilde{\varphi}_{T_{1}}, \tilde{\varphi}_{T_{2}}, \ldots, \tilde{\varphi}_{T_{\ell}}\right\} \sqcup\left\{\Delta_{j} \mid j \in J\right\}$ between

- isomorphism classes of reachable basic cluster tilting objects of Sub $Q_{J}$;

- clusters of $\tilde{\mathcal{A}}$.

Moreover, it commutes with mutation of cluster tilting objects and mutation of clusters.

6B. Categorification of the cluster algebra structure of $\mathbb{C}[\mathcal{F}]$ using $\mathrm{CM}_{e}$ A. We keep the setting of the beginning of this section, and we fix $R:=\mathbb{C} \llbracket t \rrbracket$. Our aim is to categorify $\mathbb{C}[\mathcal{F}(\Delta, J)]$ by a category $\mathrm{CM}_{e} A$, where $A$ is an $R$-order and $e \in A$ is an idempotent. We denote by $g=g_{J}$ the idempotent of $\Pi$ corresponding to the set $J$. We also define $I_{J}:=\operatorname{Hom}_{\Pi}(\Pi /(g), \Pi)$, which is the biggest ideal of $\Pi$ satisfying $g I_{J}=0$. We observe that

- injective modules corresponding to $j \in J$ in $\bmod \Pi$ and $\bmod \Pi / I_{J}$ coincide;

- $\Pi / I_{J} \in \operatorname{Sub} Q_{J} \subset \bmod \Pi / I_{J} \subset \bmod \Pi$.

We define pairs $(A, e)$ permitting the categorification.

Definition 6.7. A pair $(A, e)$, where $A$ is an $R$-order and $e \in A$ is an idempotent models $(\Delta, J)$ if

- $B:=A /(e) \cong \Pi(\Delta) / I_{J}$ as $\mathbb{C}$-algebras;

- $\operatorname{Ext}_{A}^{1}\left(\mathrm{CM}_{e} A, A e\right)=0$, that is, $(\mathrm{E} 1)$ holds, and $\operatorname{Ext}_{\bmod _{e} A}^{2}\left(\bmod _{e} A, A e\right)=0$, that is, (E2) holds;

- $B$-cotop induces a bijection from ind $A e$ to ind $\left(\operatorname{soc} Q_{J}\right)$.

Using the last condition of Definition 6.7, if $(A, e)$ models $(\Delta, J)$, we can decompose $e$ as sum of primitive orthogonal idempotents $e=\sum_{j \in J} e_{j}$ in such a way that for every $j \in J$,

$$
B \text {-cotop } A e_{j} \cong S_{j}
$$

where, as before, $S_{j}$ is soc $Q_{j}$ (not top $A e_{j}$ ).

In this context, we have the following equivalence of categories:

Lemma 6.8. If $(A, e)$ models $(\Delta, J)$, then $B \otimes_{A}-$ restricts to an exact bijective functor $F: \mathrm{CM}_{e} A \rightarrow \operatorname{Sub} Q_{J}$, which induces an equivalence of exact categories $\left(\mathrm{CM}_{e} A\right) /[A e] \rightarrow \operatorname{Sub} Q_{J}$. 
Proof. Thanks to Theorem 2.2(d) and (e), $F:=B \otimes_{A}-: \mathrm{CM}_{e} A \rightarrow$ Sub $U$ induces an equivalence of exact categories $\left(\mathrm{CM}_{e} A\right) /[A e] \rightarrow$ Sub $U$ for some injective $B$-module $U$, so $F$ is exact bijective. By Lemma 5.8(d), we have $U \cong Q_{J}$; hence the statement holds.

We start by proving the following proposition by applying the method of change of orders given in Theorem 2.3.

Proposition 6.9. Assume that $(A, e)$ models $(\Delta, J)$. Then, for any subset $J^{\prime}$ of $J$, there exists an order $A^{\prime}$, explicitly constructed from $A$, and an idempotent $e^{\prime}$ of $A^{\prime}$ such that $\left(A^{\prime}, e^{\prime}\right)$ models $\left(\Delta, J^{\prime}\right)$.

Proof. First of all, using indices of (6-1), let $e^{\prime}=\sum_{j \in J^{\prime}} e_{j}$. Define $B:=\Pi / I_{J}$ and $B^{\prime}:=\Pi / I_{J^{\prime}}$. Then $B^{\prime}$ is a quotient of $A /\left(e^{\prime}\right)$. Let us check that $\left(A, e^{\prime}\right)$ and $B^{\prime}$ satisfy the hypotheses of Theorem 2.3. First of all, (C1) is clear. By Lemma 6.8, $Q_{J^{\prime}} \cong F X$ for some $X \in \mathrm{CM}_{e} A$ without nonzero direct summands in add $A e$. Moreover, according to Proposition 5.9(a), $B$-cotop $T X \cong \operatorname{soc} Q_{J^{\prime}}$ so $T X \cong A e^{\prime}$. Therefore, thanks to Lemma 5.4, we get

$$
B^{\prime} \in \operatorname{Sub} Q_{J^{\prime}} \subset \operatorname{Sub}\left(A e^{\prime} \otimes_{R}(K / R)\right) ;
$$

hence (C2) is satisfied. It is immediate that $\mathrm{CM}_{e^{\prime}}^{B^{\prime}} A \subset \mathrm{CM}_{e} A$ so, thanks to (E1), we get $(\mathrm{C} 3) \operatorname{Ext}_{A}^{1}\left(\mathrm{CM}_{e^{\prime}}^{B^{\prime}} A, A e^{\prime}\right)=0$.

We apply Theorem 2.3 to the pair $\left(A, e^{\prime}\right)$ and $B^{\prime}$ to get an explicit order $A^{\prime}$. Let us show that $\left(A^{\prime}, e^{\prime}\right)$ models $\left(\Delta, J^{\prime}\right)$. We have $B^{\prime} \cong A^{\prime} /\left(e^{\prime}\right)$ by Theorem 2.3(a). Moreover, $\left(A^{\prime}, e^{\prime}\right)$ satisfies (E1) and (E2) $)^{+}$by Theorem 2.3(b), so it also satisfies (E2). It remains to check for $j \in J^{\prime}$ that $B^{\prime}-\operatorname{cotop}\left(A^{\prime} e_{j}\right) \cong S_{j}$. Thanks to Proposition 5.12 and Lemma 5.13, applying $H$ to $0 \rightarrow A e_{j} \rightarrow B-\operatorname{corad}\left(A e_{j}\right) \rightarrow S_{j} \rightarrow 0$ gives a short exact sequence $0 \rightarrow A^{\prime} e_{j} \rightarrow H\left(B-\operatorname{corad}\left(A e_{j}\right)\right) \rightarrow S_{j} \rightarrow 0$ which does not split. Moreover, $H\left(B-\operatorname{corad}\left(A e_{j}\right)\right) \in \mathrm{CM}_{e^{\prime}} A^{\prime}$ so $S_{j}$ is a summand of $B^{\prime}-\operatorname{cotop}\left(A^{\prime} e_{j}\right)$. So, thanks to Lemma 5.8, $B^{\prime}-\operatorname{cotop}\left(A^{\prime} e_{j}\right) \cong S_{j}$.

As a consequence, we obtain the following important result of this paper:

Theorem 6.10. For any Dynkin diagram $\Delta$ and any set $J$ of vertices of $\Delta$, there exists a pair $(A, e)$ which models $(\Delta, J)$.

Proof. As $\Pi$ is self-injective, thanks to Corollary $\mathrm{C}$, there exist an order $A$ and an idempotent $e$ of $A$ such that $A /(e) \cong \Pi$ as $\mathbb{C}$-algebras and $D_{1}(A e) \cong(1-e) A$ as right $A$-modules. So it is immediate that $(A, e)$ models $\left(\Delta, \Delta_{0}\right)$, where $\Delta_{0}$ is the set of vertices of $\Delta$. Then, Proposition 6.9 allows us to conclude immediately.

Notice that the pair $(A, e)$ in Theorem 6.10 is not unique. We will construct in [Demonet and Iyama $\geq 2016$ ] other possibilities than the one considered in this paper. 
We now fix a pair $(\Delta, J)$ and a pair $(A, e)$ modeling it. We will prove that $\mathrm{CM}_{e} A$ categorifies the cluster algebra structure of $\tilde{\mathcal{A}}$. From now on, we consider $F: \mathrm{CM}_{e} A \rightarrow \operatorname{Sub} Q_{J}$ as in Lemma 6.8. Since the category Sub $Q_{J}$ is stably 2-Calabi-Yau, $\mathrm{CM}_{e} A$ is also stably 2-Calabi-Yau. We now extend the character $\tilde{\varphi}$ to $\mathrm{CM}_{e} A$ :

Definition 6.11. For $Y \in \mathrm{CM}_{e} A$, we define $\psi_{Y} \in \tilde{\mathcal{A}}$ as follows. If $Y$ does not have nonzero direct summands in add $A e$, then $\psi_{Y}:=\tilde{\varphi}_{F Y}$. For $j \in J$, we define $\psi_{A e_{j}}:=\Delta_{j}$, and we extend the definition to $\mathrm{CM}_{e} A$ by the property $\psi_{Y \oplus Z}=\psi_{Y} \psi_{Z}$.

The following main result of this subsection improves Theorem 6.6 of Geiss, Leclerc and Schröer:

Theorem 6.12. (a) $\psi$ induces a bijection between

- isomorphism classes of reachable indecomposable rigid objects of $\mathrm{CM}_{e} A$;

- cluster variables and coefficients of $\tilde{\mathcal{A}}$.

(b) $\psi$ induces a bijection between

- isomorphism classes of reachable basic cluster tilting objects of $\mathrm{CM}_{e} A$;

- clusters of $\tilde{\mathcal{A}}$.

Moreover, it commutes with mutation of cluster tilting objects and mutation of clusters.

We start by proving that $\psi$ is a cluster character, extending Theorem 6.5:

Proposition 6.13. (a) If $Y, Z \in \mathrm{CM}_{e} A$, then $\psi_{Y \oplus Z}=\psi_{Y} \psi_{Z}$.

(b) If $Y, Z \in \mathrm{CM}_{e}$ A are indecomposable and $\operatorname{dim}_{\operatorname{Ext}_{A}^{1}}^{1}(Y, Z)=1$ (or equivalently $\left.\operatorname{dim} \operatorname{Ext}_{A}^{1}(Z, Y)=1\right)$, we have $\psi_{Y} \psi_{Z}=\psi_{U}+\psi_{U^{\prime}}$, where

$$
\xi_{1}: 0 \rightarrow Y \rightarrow U \rightarrow Z \rightarrow 0 \quad \text { and } \quad \xi_{2}: 0 \rightarrow Z \rightarrow U^{\prime} \rightarrow Y \rightarrow 0
$$

are two nonsplit short exact sequences.

We need the following lemma, stated without proof in [Geiss et al. 2008], which can also be seen as a corollary of the much more general [Geiss et al. 2011, Proposition 12.4]. For the sake of convenience, we give a direct proof.

Lemma 6.14. For any $j \in J$, at least one of the following complexes is exact:

$\operatorname{Hom}_{\Pi}\left(S_{j}, F \xi_{1}\right): 0 \rightarrow \operatorname{Hom}_{\Pi}\left(S_{j}, F Y\right) \rightarrow \operatorname{Hom}_{\Pi}\left(S_{j}, F U\right) \rightarrow \operatorname{Hom}_{\Pi}\left(S_{j}, F Z\right) \rightarrow 0$, $\operatorname{Hom}_{\Pi}\left(S_{j}, F \xi_{2}\right): 0 \rightarrow \operatorname{Hom}_{\Pi}\left(S_{j}, F Z\right) \rightarrow \operatorname{Hom}_{\Pi}\left(S_{j}, F U^{\prime}\right) \rightarrow \operatorname{Hom}_{\Pi}\left(S_{j}, F Y\right) \rightarrow 0$. Proof. Applying $F$ to $\xi_{1}$ and $\xi_{2}$, we get short exact sequences $F \xi_{1}$ and $F \xi_{2}$. Applying $\operatorname{Hom}_{\Pi}\left(S_{j},-\right)$ to $F \xi_{1}$ and $F \xi_{2}$, it is enough to show that at least one of the induced morphisms

$$
\operatorname{Hom}_{\Pi}\left(S_{j}, F Z\right) \rightarrow \operatorname{Ext}_{\Pi}^{1}\left(S_{j}, F Y\right) \quad \text { and } \operatorname{Hom}_{\Pi}\left(S_{j}, F Y\right) \rightarrow \operatorname{Ext}_{\Pi}^{1}\left(S_{j}, F Z\right)
$$


vanishes. Without loss of generality, suppose that there exists $f: S_{j} \hookrightarrow F Z$ such that the induced extension in $\operatorname{Ext}_{\Pi}^{1}\left(S_{j}, F Y\right)$ is nonzero. We deduce that

$$
\operatorname{Ext}_{\Pi}^{1}(f, F Y): \operatorname{Ext}_{\Pi}^{1}(F Z, F Y) \rightarrow \operatorname{Ext}_{\Pi}^{1}\left(S_{j}, F Y\right)
$$

is nonzero, so injective as $\operatorname{dim} \operatorname{Ext}_{\Pi}^{1}(F Z, F Y)=1$. As $\Pi$ is stably 2-Calabi-Yau, we get that

$$
\operatorname{Ext}_{\Pi}^{1}(F Y, f): \operatorname{Ext}_{\Pi}^{1}\left(F Y, S_{j}\right) \rightarrow \operatorname{Ext}_{\Pi}^{1}(F Y, F Z)
$$

is surjective, so there is a pushout diagram

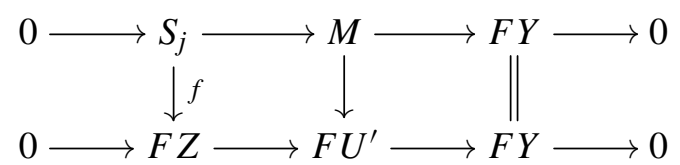

the second row of which is the image by $F$ of the short exact sequence given in Proposition 6.13(b). So, as $\operatorname{Ext}_{\Pi}^{1}\left(S_{j}, S_{j}\right)=0$, any $g: S_{j} \rightarrow F Y$ factors through $M$, and hence through $F U^{\prime}$. Therefore, the map $\operatorname{Hom}_{\Pi}\left(S_{j}, F Y\right) \rightarrow \operatorname{Ext}_{\Pi}^{1}\left(S_{j}, F Z\right)$ vanishes.

Proof of Proposition 6.13. (a) It is an obvious consequence of the property for $\tilde{\varphi}$ and our definition of $\psi$.

(b) Consider decompositions $U \cong U_{0} \oplus U_{1}$ and $U^{\prime} \cong U_{0}^{\prime} \oplus U_{1}^{\prime}$, where $U_{1}$ and $U_{1}^{\prime}$ are maximal direct summands contained in add Ae. Thanks to Proposition 5.9(b), we have

$$
U_{1}=\bigoplus_{j \in J}\left(A e_{j}\right)^{a_{j}+b_{j}-c_{j}} \quad \text { and } \quad U_{1}^{\prime}=\bigoplus_{j \in J}\left(A e_{j}\right)^{a_{j}+b_{j}-c_{j}^{\prime}}
$$

where, for $j \in J$,

- $a_{j}=\operatorname{dim} \operatorname{Hom}_{\Pi / I_{j}}\left(S_{j}, F Y\right)=\operatorname{dim} \operatorname{Hom}_{\Pi}\left(S_{j}, F Y\right)$;

- $b_{j}=\operatorname{dim} \operatorname{Hom}_{\Pi / I_{j}}\left(S_{j}, F Z\right)=\operatorname{dim} \operatorname{Hom}_{\Pi}\left(S_{j}, F Z\right)$;

- $c_{i}=\operatorname{dim} \operatorname{Hom}_{\Pi / I_{j}}\left(S_{j}, F U\right)=\operatorname{dim}_{H_{0}}\left(S_{j}, F U\right)$;

- $c_{i}^{\prime}=\operatorname{dim} \operatorname{Hom}_{\Pi / I_{j}}\left(S_{j}, F U^{\prime}\right)=\operatorname{dim} \operatorname{Hom}_{\Pi}\left(S_{j}, F U^{\prime}\right)$.

By Lemma 6.14, using the $\alpha_{j}$ and $\beta_{j}$ of Theorem 6.5, we have $a_{j}+b_{j}-c_{j}=$ $\max \left(0, c_{j}^{\prime}-c_{j}\right)=\alpha_{j}$ and $a_{j}+b_{j}-c_{j}^{\prime}=\beta_{j}$. Thus, Theorem 6.5 implies

$$
\begin{aligned}
\psi_{Y} \psi_{Z}=\tilde{\varphi}_{F Y} \tilde{\varphi}_{F Z} & =\tilde{\varphi}_{F U} \prod_{j \in J} \Delta_{j}^{\alpha_{j}}+\tilde{\varphi}_{F U^{\prime}} \prod_{j \in J} \Delta_{j}^{\beta_{j}} \\
& =\psi_{U_{0}} \psi_{U_{1}}+\psi_{U_{0}^{\prime}} \psi_{U_{1}^{\prime}}=\psi_{U}+\psi_{U^{\prime}}
\end{aligned}
$$

Now, we can deduce the proof of Theorem 6.12: 
Proof of Theorem 6.12. By Theorem 6.6, it is enough to note that $F: \mathrm{CM}_{e} A \rightarrow \operatorname{Sub} U$ induces a bijection between isomorphism classes of basic cluster tilting objects. This is immediate as $F$ induces a triangle equivalence $\underline{\mathrm{CM}}_{e} A \cong \underline{\mathrm{Sub}} U$. More precisely, basic cluster tilting objects of $\mathrm{CM}_{e} A$ are of the form $A e \oplus T$, where $T$ has no direct summand in add $A e$, and the indecomposable direct summands of $T$ correspond bijectively to the indecomposable direct summands of $F T$.

\section{Acknowledgement}

We would like to thank Alastair King and Bernard Leclerc for valuable discussion about this topic.

\section{References}

[Amiot et al. 2015] C. Amiot, O. Iyama, and I. Reiten, "Stable categories of Cohen-Macaulay modules and cluster categories", Amer. J. Math. 137:3 (2015), 813-857. MR 3357123 Zbl 06458529

[Araya 1999] T. Araya, "Exceptional sequences over graded Cohen-Macaulay rings", Math. J. Okayama Univ. 41 (1999), 81-102. MR 1816620 Zbl 0981.16016

[Auslander 1978] M. Auslander, "Functors and morphisms determined by objects", pp. 1-244 in Representation theory of algebras (Philadelphia, PA, 1976), edited by R. Gordon, Lecture Notes in Pure Appl. Math. 37, Dekker, New York, 1978. MR 0480688 Zbl 0383.16015

[Auslander and Reiten 1991] M. Auslander and I. Reiten, "Applications of contravariantly finite subcategories”, Adv. Math. 86:1 (1991), 111-152. MR 1097029 Zbl 0774.16006

[Baur et al. 2016] K. Baur, A. King, and R. J. Marsh, "Dimer models and cluster categories of Grassmannians", Proc. London Math. Soc. 113:2 (2016), 213-260.

[Berenstein et al. 2005] A. Berenstein, S. Fomin, and A. Zelevinsky, "Cluster algebras, III: Upper bounds and double Bruhat cells", Duke Math. J. 126:1 (2005), 1-52. MR 2110627 Zbl 1135.16013

[Borel 1991] A. Borel, Linear algebraic groups, 2nd ed., Graduate Texts in Mathematics 126, Springer, New York, 1991. MR 1102012 Zbl 0726.20030

[Chen 2012] X.-W. Chen, “Three results on Frobenius categories”, Math. Z. 270:1-2 (2012), 43-58. MR 2875822 Zbl 1244.18004

[Curtis and Reiner 1981] C. W. Curtis and I. Reiner, Methods of representation theory, I: With applications to finite groups and orders, Wiley, New York, 1981. MR 632548 Zbl 0469.20001

[Demonet and Iyama $\geq 2016$ ] L. Demonet and O. Iyama, "Idempotent-projective Cohen-Macaulay modules and their Auslander-Reiten quiver", work in progress.

[Demonet and Luo 2016a] L. Demonet and X. Luo, "Ice quivers with potential arising from oncepunctured polygons and Cohen-Macaulay modules", Publ. Res. Inst. Math. Sci. 52:2 (2016), 141-205. MR 3490479 Zbl 06588940

[Demonet and Luo 2016b] L. Demonet and X. Luo, "Ice quivers with potential associated with triangulations and Cohen-Macaulay modules over orders”, Trans. Amer. Math. Soc. 368:6 (2016), 4257-4293. MR 3453371 Zbl 06551092

[Fomin and Zelevinsky 1999] S. Fomin and A. Zelevinsky, "Double Bruhat cells and total positivity", J. Amer. Math. Soc. 12:2 (1999), 335-380. MR 1652878 Zbl 0913.22011

[Fomin and Zelevinsky 2003] S. Fomin and A. Zelevinsky, "Cluster algebras, II: Finite type classification”, Invent. Math. 154:1 (2003), 63-121. MR 2004457 Zbl 1054.17024 
[Geiss et al. 2005] C. Geiss, B. Leclerc, and J. Schröer, "Semicanonical bases and preprojective algebras”, Ann. Sci. École Norm. Sup. (4) 38:2 (2005), 193-253. MR 2144987 Zbl 1131.17006

[Geiss et al. 2008] C. Geiss, B. Leclerc, and J. Schröer, "Partial flag varieties and preprojective algebras", Ann. Inst. Fourier (Grenoble) 58:3 (2008), 825-876. MR 2427512 Zbl 1151.16009

[Geiss et al. 2011] C. Geiss, B. Leclerc, and J. Schröer, "Kac-Moody groups and cluster algebras", Adv. Math. 228:1 (2011), 329-433. MR 2822235 Zbl 1232.17035

[Herschend et al. 2014] M. Herschend, O. Iyama, H. Minamoto, and S. Oppermann, "Representation theory of Geigle-Lenzing complete intersections", preprint, 2014. arXiv 1409.0668

[Iyama and Takahashi 2013] O. Iyama and R. Takahashi, "Tilting and cluster tilting for quotient singularities”, Math. Ann. 356:3 (2013), 1065-1105. MR 3063907 Zbl 06181402

[Jensen et al. 2016] B. T. Jensen, A. King, and X. Su, "A categorification of Grassmannian cluster algebras", Proc. London Soc. 113:2 (2016), 185-212.

[Kajiura et al. 2007] H. Kajiura, K. Saito, and A. Takahashi, "Matrix factorizations and representations of quivers, II: Type ADE case”, Adv. Math. 211:1 (2007), 327-362. MR 2313537 Zbl 1167.16011

[Kajiura et al. 2009] H. Kajiura, K. Saito, and A. Takahashi, "Triangulated categories of matrix factorizations for regular systems of weights with $\epsilon=-1$ ", Adv. Math. 220:5 (2009), 1602-1654. MR 2493621 Zbl 1172.18002

[Keller 1990] B. Keller, "Chain complexes and stable categories", Manuscripta Math. 67:4 (1990), 379-417. MR 1052551 Zbl 0753.18005

[Keller and Reiten 2008] B. Keller and I. Reiten, "Acyclic Calabi-Yau categories", Compos. Math. 144:5 (2008), 1332-1348. MR 2457529 Zbl 1171.18008

[Lakshmibai and Gonciulea 2001] V. Lakshmibai and N. Gonciulea, Flag varieties, Travaux en Cours 63, Hermann, Paris, 2001. Zbl 1136.14306

[Leuschke and Wiegand 2012] G. J. Leuschke and R. Wiegand, Cohen-Macaulay representations, Mathematical Surveys and Monographs 181, American Mathematical Society, Providence, RI, 2012. MR 2919145 Zbl 1252.13001

[Lusztig 2000] G. Lusztig, "Semicanonical bases arising from enveloping algebras", Adv. Math. 151:2 (2000), 129-139. MR 1758244 Zbl 0983.17009

[Quillen 1973] D. Quillen, "Higher algebraic K-theory, I", pp. 85-147 in Algebraic K-theory, I: Higher K-theories (Seattle, WA, 1972), Lecture Notes in Math. 341, Springer, Berlin, 1973. MR 0338129 Zbl 0292.18004

[Scott 2006] J. S. Scott, "Grassmannians and cluster algebras”, Proc. London Math. Soc. (3) 92:2 (2006), 345-380. MR 2205721 Zbl 1088.22009

[Simson 1992] D. Simson, Linear representations of partially ordered sets and vector space categories, Algebra, Logic and Applications 4, Gordon and Breach Science Publishers, Montreux, 1992. MR 1241646 Zbl 0818.16009

[de Thanhoffer de Völcsey and Van den Bergh 2010] L. de Thanhoffer de Völcsey and M. Van den Bergh, "Explicit models for some stable categories of maximal Cohen-Macaulay modules", preprint, 2010. To appear in Math. Res. Lett. arXiv 1006.2021

[Yoshino 1990] Y. Yoshino, Cohen-Macaulay modules over Cohen-Macaulay rings, London Mathematical Society Lecture Note Series 146, Cambridge University Press, 1990. MR 1079937 Zbl 0745.13003

Communicated by Sergey Fomin

Received 2015-09-25 Revised 2016-04-26 
laurent.demonet@normalesup.org

iyama@math.nagoya-u.ac.jp
Graduate School of Mathematics, Nagoya University, Furocho, Chikusaku, Nagoya 464-8602, Japan

Graduate School of Mathematics, Nagoya University, Furocho, Chikusaku, Nagoya 464-8602, Japan 


\section{Algebra \& Number Theory}

msp.org/ant

\section{EDITORS}

MANAGING EDITOR

Bjorn Poonen

Massachusetts Institute of Technology

Cambridge, USA

\author{
EDITORIAL BOARD CHAIR \\ David Eisenbud \\ University of California \\ Berkeley, USA
}

BOARD OF EDITORS

$\begin{aligned} \text { Dave Benson } & \text { University of Aberdeen, Scotland } & \text { Susan Montgomery } & \text { University of Southern California, USA } \\ \text { Richard E. Borcherds } & \text { University of California, Berkeley, USA } & \text { Shigefumi Mori } & \text { RIMS, Kyoto University, Japan } \\ \text { John H. Coates } & \text { University of Cambridge, UK } & \text { Raman Parimala } & \text { Emory University, USA } \\ \text { J-L. Colliot-Thélène } & \text { CNRS, Université Paris-Sud, France } & \text { Jonathan Pila } & \text { University of Oxford, UK } \\ \text { Brian D. Conrad } & \text { Stanford University, USA } & \text { Anand Pillay } & \text { University of Notre Dame, USA } \\ \text { Hélène Esnault } & \text { Freie Universität Berlin, Germany } & \text { Victor Reiner } & \text { University of Minnesota, USA } \\ \text { Hubert Flenner } & \text { Ruhr-Universität, Germany } & \text { Peter Sarnak } & \text { Princeton University, USA } \\ \text { Sergey Fomin } & \text { University of Michigan, USA } & \text { Joseph H. Silverman } & \text { Brown University, USA } \\ \text { Edward Frenkel } & \text { University of California, Berkeley, USA } & \text { Michael Singer } & \text { North Carolina State University, USA } \\ \text { Andrew Granville } & \text { Université de Montréal, Canada } & \text { Vasudevan Srinivas } & \text { Tata Inst. of Fund. Research, India } \\ \text { Joseph Gubeladze } & \text { San Francisco State University, USA } & \text { J. Toby Stafford } & \text { University of Michigan, USA } \\ \text { Roger Heath-Brown } & \text { Oxford University, UK } & \text { Ravi Vakil } & \text { Stanford University, USA } \\ \text { Craig Huneke } & \text { University of Virginia, USA } & \text { Michel van den Bergh } & \text { Hasselt University, Belgium } \\ \text { Kiran S. Kedlaya } & \text { Univ. of California, San Diego, USA } & \text { Marie-France Vignéras } & \text { Université Paris VII, France } \\ \text { János Kollár } & \text { Princeton University, USA } & \text { Kei-Ichi Watanabe } & \text { Nihon University, Japan } \\ \text { Yuri Manin } & \text { Northwestern University, USA } & \text { Efim Zelmanov } & \text { University of California, San Diego, USA } \\ \text { Philippe Michel } & \text { École Polytechnique Fédérale de Lausanne } & \text { Shou-Wu Zhang } & \text { Princeton University, USA }\end{aligned}$

PRODUCTION

production@msp.org

Silvio Levy, Scientific Editor

See inside back cover or msp.org/ant for submission instructions.

The subscription price for 2016 is US $\$ 290$ /year for the electronic version, and $\$ 485 /$ year (+\$55, if shipping outside the US) for print and electronic. Subscriptions, requests for back issues and changes of subscribers address should be sent to MSP.

Algebra \& Number Theory (ISSN 1944-7833 electronic, 1937-0652 printed) at Mathematical Sciences Publishers, 798 Evans Hall \#3840, c/o University of California, Berkeley, CA 94720-3840 is published continuously online. Periodical rate postage paid at Berkeley, CA 94704, and additional mailing offices.

ANT peer review and production are managed by EditFLow ${ }^{\circledR}$ from MSP.

\section{PUBLISHED BY}

- mathematical sciences publishers

nonprofit scientific publishing

http://msp.org/

() 2016 Mathematical Sciences Publishers 


\section{Algebra \& Number Theory}

$\begin{array}{lll}\text { Volume } 10 & \text { No. } 7 \quad 2016\end{array}$

Arithmetic invariant theory and 2-descent for plane quartic curves JACK A. THORNE

Furstenberg sets and Furstenberg schemes over finite fields JORDAN S. ELLENBERG and DANIEL ERMAN

Local deformation rings for $\mathrm{GL}_{2}$ and a Breuil-Mézard conjecture when $l \neq p$ JACK SHOTTON

Generalized Kuga-Satake theory and rigid local systems, II: rigid Hecke eigensheaves STEFAN PATRIKIS

Lifting preprojective algebras to orders and categorifying partial flag varieties

LAURENT DEMONET and OSAMU IYAMA

A fibered power theorem for pairs of log general type 